Florida International University FIU Digital Commons

\title{
An Autoregressive Conditional Filtering Process to remove Intraday Seasonal Volatility and its Application to Testing the Noisy Rational Expectations Model
}

Jang Hyung Cho

Florida International University

DOI: $10.25148 /$ etd.FI08121903

Follow this and additional works at: https://digitalcommons.fiu.edu/etd

Part of the Finance and Financial Management Commons

\section{Recommended Citation}

Cho, Jang Hyung, "An Autoregressive Conditional Filtering Process to remove Intraday Seasonal Volatility and its Application to Testing the Noisy Rational Expectations Model" (2008). FIU Electronic Theses and Dissertations. 60.

https://digitalcommons.fiu.edu/etd/60 


\title{
FLORIDA INTERNATIONAL UNIVERSITY \\ Miami, Florida
}

\begin{abstract}
AN AUTOREGRESSIVE CONDITIONAL FILTERING PROCESS TO REMOVE INTRADAY SEASONAL VOLATILITY AND ITS APPLICATION TO TESTING

THE NOISY RATIONAL EXPECTATIONS MODEL
\end{abstract}

\author{
A dissertation submitted in partial fulfillment of the \\ requirements for the degree of \\ DOCTOR OF PHILOSOPHY \\ in \\ BUSINESS ADMINISTRATION \\ by \\ Jang Hyung Cho
}

2008 
To: Dean Joyce Elam

College of Business Administration

This dissertation, written by Jang Hyung Cho, and entitled An Autoregressive Conditional Filtering Process to Remove Intraday Seasonal Volatility and Its Application to Testing the Noisy Rational Expectations Model, having been approved in respect to style and intellectual content, is referred to you for judgment.

We have read this dissertation and recommend that it be approved.

Ali M. Parhizgari

Brice Dupoyet

Xiaoquan Jiang

Krishnamurthy Surysekar

Robert T. Daigler, Major Professor

Date of Defense: July 15, 2008

The dissertation of Jang Hyung Cho is approved.

Dean Joyce Elam
College of Business Administration

Florida International University, 2008 
(C) Copyright 2008 by Jang Hyung Cho

All rights reserved. 


\section{ACKNOWLEDGMENTS}

I would like to thank my dissertation committee members: Dr. Robert T. Daigler, Dr. Ali Parhizgari, Dr. Brice Dupoyet, Dr. Xiaoquan Jiang, and Dr. Krishnamurthy Surysekar. Especially, I would like to express my sincere gratitude to Dr. Robert T. Daigler. Throughout my doctoral studies, he continuously encouraged me to develop research skills and independent thinking. He also assisted me with scientific writing.

I wish to thank my loving wife and family for their continual encouragements

throughout my doctoral studies. My sincere thanks to God for his immeasurable, unconditional love and grace. 


\begin{abstract}
OF THE DISSERTATION
AN AUTOREGRESSIVE CONDITIONAL FILTERING PROCESS TO REMOVE

INTRADAY SEASONAL VOLATILITY AND ITS APPLICATION TO TESTING

THE NOISY RATIONAL EXPECTATIONS MODEL
\end{abstract}

by

Jang Hyung Cho

Florida International University, 2008

Miami, Florida

Professor Robert T. Daigler, Major Professor

We develop a new autoregressive conditional process to capture both the changes and the persistency of the intraday seasonal (U-shape) pattern of volatility in essay 1. Unlike other procedures, this approach allows for the intraday volatility pattern to change over time without the filtering process injecting a spurious pattern of noise into the filtered series. We show that prior deterministic filtering procedures are special cases of the autoregressive conditional filtering process presented here. Lagrange multiplier tests prove that the stochastic seasonal variance component is statistically significant. Specification tests using the correlogram and cross-spectral analyses prove the reliability of the autoregressive conditional filtering process.

In essay 2 we develop a new methodology to decompose return variance in order to examine the informativeness embedded in the return series. The variance is decomposed into the information arrival component and the noise factor component. This decomposition methodology differs from previous studies in that both the informational variance and the noise variance are time-varying. Furthermore, the covariance of the 
informational component and the noisy component is no longer restricted to be zero. The resultant measure of price informativeness is defined as the informational variance divided by the total variance of the returns.

The noisy rational expectations model predicts that uninformed traders react to price changes more than informed traders, since uninformed traders cannot distinguish between price changes caused by information arrivals and price changes caused by noise. This hypothesis is tested in essay 3 using intraday data with the intraday seasonal volatility component removed, as based on the procedure in the first essay. The resultant seasonally adjusted variance series is decomposed into components caused by unexpected information arrivals and by noise in order to examine informativeness. 


\section{TABLE OF CONTENTS}

ESSAY

PAGE

ESSAY 1. An Autoregressive Conditional Filtering Process to Remove Intraday

Seasonal Volatility …………………......................................................

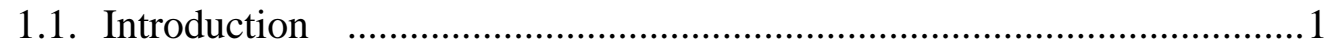

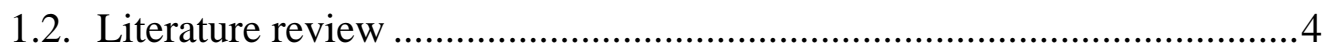

1.3. Modeling the dynamics of intraday seasonal volatility ............................10

1.4. Gains in filtering performance of ARCSV process....................................14

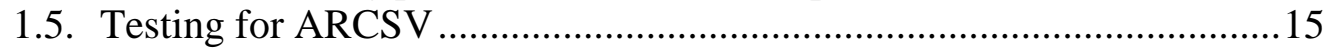

1.6. Testing for specification and model selection ..........................................17

1.6.1. Correlogram tests for the seasonal volatility at seasonal lags .........18

1.6.2. Correlogram tests for the performance of the ARCSV process .....18

1.6.3. Cross-spectral tests .........................................................................20

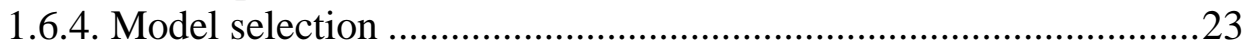

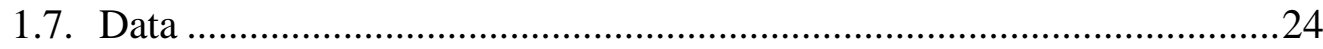

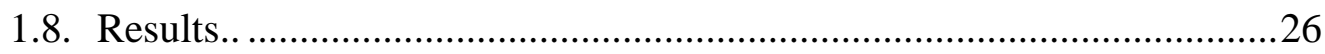

1.8.1. Correlogram test results of the seasonal volatility at seasonal

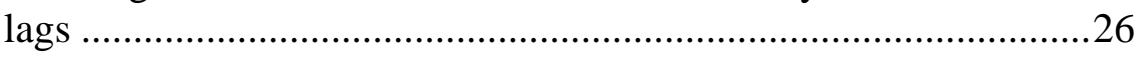

1.8.2. Estimation results of the ARCSV models .......................................27

1.8.3. Correlogram test results of the performance of the filtering

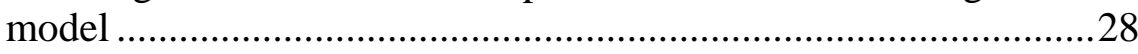

1.8.4. Cross-spectral test results of the performance of the filtering

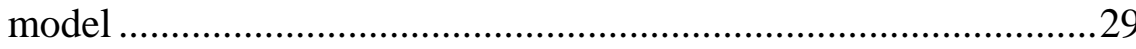

1.8.5. Results of the in-sample fit............................................................32

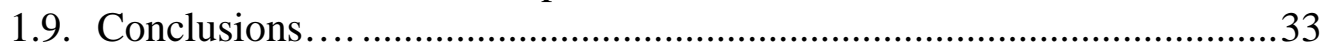

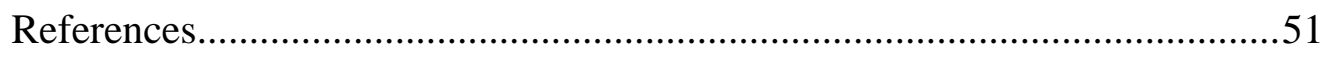

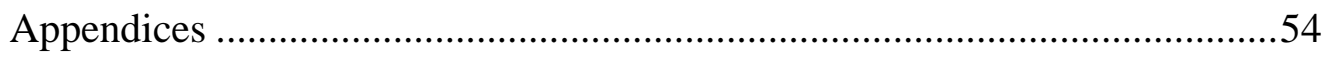

ESSAY 2. Informational Decompositions of the Variance of Returns ........................69

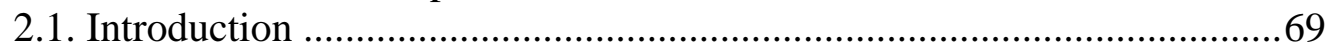

2.2. Concepts and literature review ................................................................71

2.3. Models for variance decomposition.............................................................73

2.3.1. Heteroskedastic variance decomposition by information content and development of a time-varying measure of price

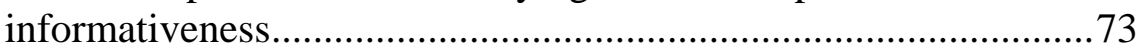

2.3.2. Testing for price informativeness .................................................. 80

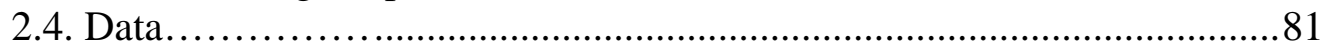

2.5. Results of variance decomposition by information and noise.............82

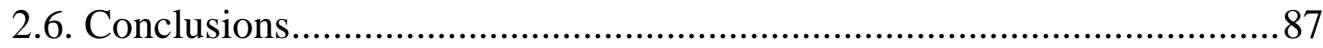

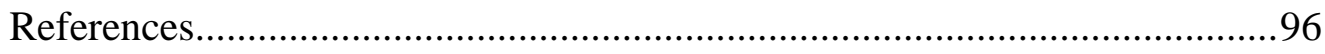

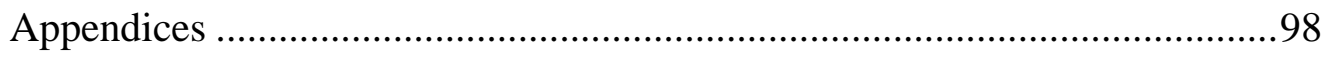


ESSAY 3. Intraday Tests of the Noisy Rational Expectations Information Model....102

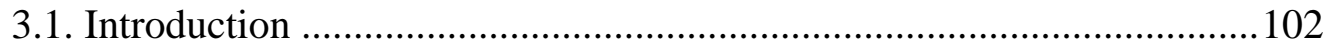

3.2. Concepts and literature review ............................................................103

3.3. Hypotheses and empirical methodologies ..............................................107

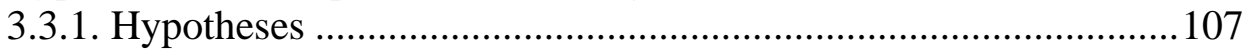

3.3.2. Empirical models for tests of the noisy rational expectations

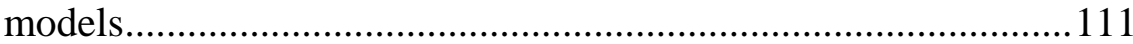

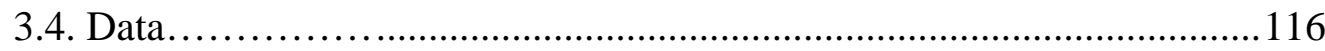

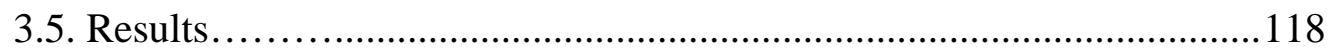

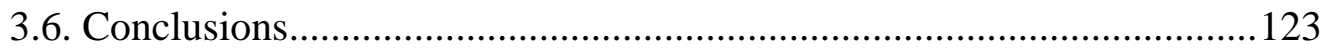

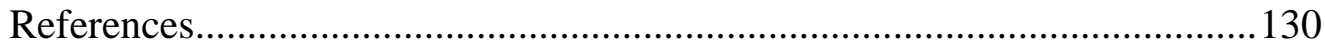

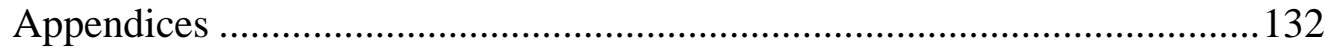

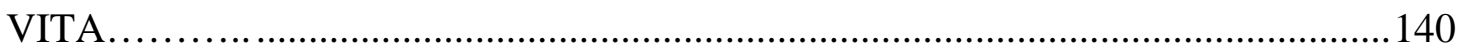




\section{LIST OF TABLES}

TABLE

PAGE

1.1. Estimation and Test Results of the ARCSV Process...................................40

1.2. Estimation Results of the Flexible Fourier Form (FFF) Approach ...............41

1.3. Estimation results of in-sample fit and encompassing regressions ................50

2.1. Daily Estimation Results of Variance Decomposition by Information ..........89

2.2. Correlation Coefficients between Variance Components Decomposed into Information and Noise ....................................................................95

3.1. Correlations Coefficients between the Non-seasonal Intraday Returns and Net Trading Volumes by CTI .............................................................126

3.2. Regression Results of the Noisy Rational Expectations Models .............127

3.3. Test Results for the Noisy Rational Expectations Model .....................129 


\section{LIST OF FIGURES}

FIGURE

PAGE

1.1. Correlograms at five minute lags for the unfiltered and filtered absolute returns

1.2. Spectral density of the intraday seasonal variance estimated by the ARCSV

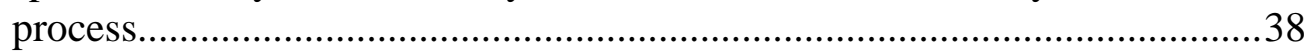

1.3. Correlogram of the normalized absolute returns at seasonal lags .................39

1.4. Intraday seasonal variance estimated by the ARCSV process ......................42

1.5. Correlograms of the seasonal variances estimated by the ARCSV

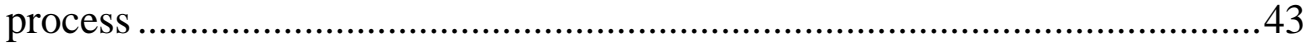

1.6. Spectral density of the estimated intraday seasonal variance ........................44

1.7. Spectral density of the unfiltered and filtered absolute returns .....................45

1.8. Estimated coherence of the unfiltered and filtered absolute returns and their 99\% confidence intervals.................................................................46

1.9. Estimated phase and $99 \%$ confidence intervals of the unfiltered and filtered

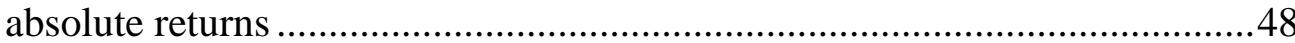

2.1. Estimated Time-varying Moving Average Coefficients ................................90

2.2. Estimated Informational Variances......................................................92

2.3. Estimated Measure of Noise Variances .......................................................93

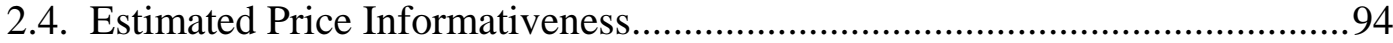




\section{Essay 1}

\section{An Autoregressive Conditional Filtering Process to Remove Intraday Seasonal Volatility}

\subsection{Introduction}

The existence of intraday seasonality (the "U-shaped" curve) makes the decomposition of total volatility into the components of volatility more difficult. ${ }^{1}$ Here I propose a new method to remove the intraday seasonality pattern to provide a better behaving filtered series to explain how intraday volatility actually changes over time, exclusive of the U-shape seasonality factor. Moreover, the current methods to estimate and remove the intraday seasonality factor actually cause a noise pattern to be embedded into the remaining filtered volatility series and do not capture the stochastic component of seasonality factor. This new method avoids such problems.

Various models are developed to filter the seasonal variance component. The models in the literature disentangle intraday seasonality from total volatility by using a new time scale (Dacorogna, Müller, Nagler, Olsen, and Pictet ,1993), the flexible Fourier form approach (Andersen and Bollerslev, 1997a), the low-pass filtering method in conjunction with the Fourier transform procedure (Andersen and Bollerslev, 1997b), the low-pass filtering method in conjunction with the discrete wavelet transform procedure (Gençay, Selçuk and Whitcher, 2001), the stochastic volatility model (Beltratti and Morana, 2001), the method of the means of the squared normalized returns for each intraday interval (Andersen, Bollerslev, Diebold and Labys, 2003; Engle, Sokalska and

\footnotetext{
${ }^{1}$ See Wood, McInish and Ord (1985), Lockwood and Linn (1990), and Daigler (1997) for an examination of intraday seasonal volatility for high frequency time series.
} 
Chanda, 2006), dummy variables (Hughes and Winters, 2005), and a neural network approach (Omrane and Bodt, 2007).

The approaches employed by Dacorogna, et al. (1993), Andersen and Bollerslev (1997a), Hughes and Winters (2001), Andersen, Bollerslev, Diebold and Labys (2003) and Engle, et al. (2006) assume that the intraday seasonal volatility is deterministic. However, the seasonal variance may contain the stochastic component as well as the deterministic component. ${ }^{2}$ Hence, if the intraday seasonal volatility pattern is timevarying then using a deterministically-fitted curve will not capture the seasonal variance perfectly, and any modification of the pure deterministic filtering models without allowing for innovation in the seasonal pattern to capture the time-varying seasonal pattern will lead to statistical distortion. In particular, the flexible Fourier form (FFF) approach injects noise into the filtered time series if the interaction terms between the daily volatility and the sinusoid terms are included to help capture the time-varying seasonal pattern. Alternatively, the low-pass filtering methods with the Fourier transform and with the wavelet transform procedures eliminate the entire intraday volatility component as part of the analysis, not just the intraday seasonality factor. Hence, these latter methods preclude the possibility of analyzing the non-seasonal intraday volatility behavior. Beltratti and Morana (2001) and Omrane and Bodt (2007) capture the stochastic component of the seasonal variance. However, Beltratti and Morana's model still requires the deterministic dynamics of the stochastic seasonal component with a complex estimation procedure. Omrane and Bodt's model does not provide the dynamics

\footnotetext{
${ }^{2}$ See Harvey (1981), Hylleberg (1986), Andersen and Bollerslev (1997a), Beltratti and Morana (2001), and Omrane and Bodt (2007) for the discussion of the stochastic component of the seasonal variance.
} 
of the seasonal variance, and the filtered series by the neural variance network method still exhibit seasonal behavior.

My model overcomes the difficulties of these previous models by assuming that the intraday seasonal variance in each season follows a unique autoregressive moving average (ARMA) process. This approach allows one to capture both the deterministic seasonal component and the changes and the persistency in the seasonal pattern, resulting in an increase in fitting the intraday seasonal volatility. Moreover, this method does not cause any misleading statistical inferences due to the injection of additional noise into the filtered series by the filtering process. My model also keeps the short-run non-seasonal intraday volatility behavior as a separate factor, since it filters out only the seasonal component at the seasonal frequencies, without removing the volatility at the nonseasonal frequencies. In addition, the estimation procedure is far simpler than the prior filtering models. Filtering is performed by univariate maximum likelihood estimations. Specification tests using the correlogram and cross-spectral analyses prove the reliability of this new autoregressive seasonal variance (ARCSV) filtering process. This paper also proves that the prior deterministic filtering models given in Andersen and Bollerslev (1997a), Andersen, et al. (2003) and Engle, et al. (2006) are special cases of the ARCSV filtering process developed in this paper.

The results show that the ARCSV filtering process performs very well. In particular, the process does not produce any distortions in the harmonic properties of the time series, such as power spectrum or phase relations at each frequency. Lagrange multiplier tests prove that there are statistically significant stochastic component in the seasonal variance. The new ARCSV filtering process shows a better filtering performance than the prior 
deterministic filtering procedures by capturing the innovation and persistency of the seasonal volatility (which account for $10.15 \%$ of the total seasonal volatility), as well as the traditional deterministic unconditional mean factor of seasonal volatility (which accounts for $89.85 \%$ of the seasonal volatility rather than the typical $100 \%$ ) for S\&P 500 futures, live cattle futures, and the JPY-USD spot exchange rate.

Section 1.2 discusses the literature on filtering intraday seasonal volatility. Section 1.3 develops a new autoregressive seasonal variance filtering process. In Section 1.4, gains of filtering efficiency from the autoregressive seasonal variance filtering process In Section 1.5, a test statistic of whether the stochastic seasonal variance component is significant is developed. In Section 1.6, pecification tests utilizing the correlogram and spectral analysis are introduced. Section 1.7 describes the data used in this study. Results are reported in section 1.8. The conclusions follow in section 1.9.

\subsection{Literature Review}

There are many reasons why the seasonal variance component has to be filtered from the total variance series. On the condition that there is a seasonal variance component in the total variance,

a. Estimated coefficients of any variance model are biased if the variance model is not adjusted for the seasonal variance. (Omrane and Bodt, 2007)

b. The time series with seasonally varying mean and variance is nonstationary. (Lutkepohle, 2007) 
c. Only non-seasonal variance component can reflect the effect of non-seasonal information arrivals. In other words, failure to adjust for them can result in misleading statistical analysis. (Goodhart and O’Hara, 1997)

d. Correlation-based measures for the degree of volatility persistence obtained from high frequency intraday data are dominated by the effect of strong periodic component. (Andersen and Bollerslev, 1997b)

e. Standard GARCH models by themselves require a geometric decay in the autocorrelation structure of volatility, and therefore cannot accommodate the volatility process which possesses a strong regular cyclical pattern in its autocorrelation structure, arising from the intraday seasonal volatility component. ${ }^{3}$ Hence, if standard GARCH models are used without adjusting for intraday seasonal volatility then any statistical inferences based on this modeling are misleading. (Andersen and Bollerslev, 1997a; Engle et al., 2006)

Andersen and Bollerslev (1997a) show the persistence of intraday seasonal volatility using the DM-USD exchange rates and S\&P 500 index futures, where the averaged absolute returns exhibit a strong U-shape pattern, and the correlograms of the absolute returns of the two series show a regular fluctuation on a daily basis. Since GARCH models are misspecified when a seasonal fluctuation in the volatility correlogram exists, Andersen and Bollerslev employ the flexible Fourier form (FFF) approach to filter out the intraday seasonal volatility component from the return series. ${ }^{4}$

\footnotetext{
${ }^{3}$ The proof of the geometric decay in the volatility correlogram of the GARCH model is found in Bollerslev (1986, p. 313-314) and Ding and Granger (1996, p. 193).

${ }^{4}$ Their resultant filtered series is based on the assumption that the conditional volatility is a multiplicative product of the non-seasonal daily volatility and the intraday seasonal volatility.
} 
The fitted seasonal pattern removed by the FFF method for the period of a day is deterministic in that the FFF method does not include a stochastic innovation process in its procedure to remove the seasonal pattern. The FFF model is partly able to capture the time-varying seasonal pattern by using the interaction terms between the exogenous daily volatility and the sinusoid terms. However, it is shown in this paper that the interaction terms that is designed to capture the time-varying seasonal pattern induce the FFF model to inject an additional noise into the filtered returns. (see Lemma 1 in Appendix 4) In other words, as the seasonal pattern becomes more time varying, the FFF filtering approach injects a larger noise component into the filtered returns through the interaction terms. ${ }^{5}$ Meanwhile, the pure deterministic FFF model still leave the stochastic seasonal component in the filtered returns. ${ }^{6}$

Andersen, Bollerslev, Diebold and Labys (2003) and Engle, Sokalska and Chanda (2006) compute the seasonal volatility (variance) is defined as the mean of the

\footnotetext{
${ }^{5}$ This FFF model creates a statistical noise into the S\&P 500 index futures. These results occur because the FFF model used to filter the seasonal variance of the S\&P 500 includes the interaction terms between the daily volatility and the sinusoid terms. Since the daily volatility is not the seasonal component the fitted curve by the FFF contains the non-seasonal component. Consequently, the filtered returns will contains noise from the FFF filtering procedure. (see Andersen and Bollerselv, 1997a, p. 148 for the filtering results of the S\&P 500)

${ }^{6}$ Bollerslev and Ghysels (1996) develop a new ARCH model namely, Periodic GARCH or PGARCH, to adjust the conditional volatility for the periodic volatility component. For simplicity, PGARCH(1,1) is as follows: $E\left[\varepsilon_{t} \mid \Omega_{\tau-1}^{n}\right]=0$ and $E\left[\varepsilon_{\tau}^{2} \mid \Omega_{\tau-1}^{n}\right] \equiv \sigma_{\tau}^{2}=\omega_{n(\tau)}+\alpha_{n(\tau)} \varepsilon_{\tau-1}^{2}+\beta_{n(\tau)} \sigma_{\tau-1}^{2}$ where $\tau=N(t-1)+n$ is the cumulative intraday index, $n$ is the intraday index (or stage), and $n=1, \ldots, N$, with $N$ being the number of intraday intervals in a day. Dummy variables and sinusoids can be used for the seasonal period specific coefficients (see Martens, Chang, and Taylor, 2002). The PGARCH model is efficient in describing conditional heteroskedasticity when the seasonal volatility component is present together with the nonseasonal volatility component. However, unlike the formulation for the conditional volatility in Andersen and Bollerslev (1997a), the volatility formulation in PGARCH is unable to split the periodic (seasonal) volatility component from the non-periodic (non-seasonal) volatility component. Hence, in this study, the PGARCH model is not considered as a process that filters out the seasonality in volatility. Martens, et al. (2002) provide empirical results that the PGARCH model is more efficient in forecasting intraday volatility than is the FFF model when intraday seasonal volatility is present. Note that Martens, et al. (2002) do not compare the PGARCH to the FFF as filtering models.
} 
normalized absolute returns (the mean of the squared normalized returns) for each intraday period. ${ }^{7}$ These normalized returns are defined as returns divided by daily volatility. This intraday seasonal volatility pattern is assumed to be deterministic, since the mean of the squared normalized returns for a given intraday interval is the same for all days. ${ }^{8}$ However, if the intraday seasonal volatility pattern is time-varying in terms of the innovation factor in the seasonal pattern, then the deterministically-fitted curve leave the stochastic variance component in the filtered variance series.

Dacorogna, Müller, Nagler, Olsen, and Pictet (1993) filter out the seasonal volatility in foreign exchange markets by developing a new time scale, called a $\vartheta$-scale, that replaces the physical time scale. ${ }^{9}$ The volatility measure in the $\vartheta$-scale does not exhibit seasonal behavior. In other words, the price change $\Delta p(\vartheta(t))$ does not exhibit seasonality even though $\Delta p(t)$ does. The $\vartheta$ time change is defined as $\Delta \vartheta_{i}=\left(c^{-1}\left|\overline{\Delta p_{i} \mid}\right|\right)^{E}$,

\footnotetext{
${ }^{7}$ In this study, the approach to estimate the seasonal variance by computing the mean of the squared normalized returns, as in Andersen, Bollerslev, Diebold and Labys (2003) and Engle, et al. (2006), is interchangeably referred to as the mean of the squared normalized returns and the variance mean filtering model.

${ }^{8}$ As in Andersen and Bollerslev (1997a), a practical estimation of volatility components is done by the following two-step procedure: In the first step, the intraday seasonal variance components $s_{t, n}$ are obtained. In the second step, using the estimates of the seasonal components and the given forecasts of the daily variance components, $\hat{\sigma}_{t}^{2}$, the following $\operatorname{GARCH}(1,1)$ model is employed to estimate the intraday conditional variance component, $q_{t, n}$.

$$
R_{t, n}=\sqrt{\hat{\sigma}_{t}^{2} \hat{s}_{t, n} q_{t, n}} \varepsilon_{t, n} \text { with } q_{t, n}=\alpha_{0}+\alpha_{1}\left(\varepsilon_{t-1}^{2} / \hat{\sigma}_{t}^{2} \hat{s}_{t, n}\right)+\beta_{1} q_{t-1, n} .
$$

Because the total conditional volatility is assumed to be a multiplicative product of the daily volatility, intraday seasonal volatility, and intraday conditional volatility, and because the daily and intraday seasonal volatilities are given as exogenous values, the intraday non-seasonal conditional volatility is determined as the remaining unknown factor.

${ }^{9}$ Dacorogna,et al. (1993) show that the seasonal volatility pattern of the DM-USD exchange rate is the combination of three U-shaped seasonal patterns consisting of the Far East Asia market, the European market, and the U.S. market.
} 
where $\Delta p_{i}$ is the price change recorded at the physical time interval $i, i=1, \ldots, N$, where $N$ is the length of the seasonal period (a day or a week), $c$ is a constant specific to exchange rates, and $E$ is a value of $1 / 0.6 .{ }^{10}$ The $\vartheta$ time interval is the monotonic transform of the average of the price change at the physical time interval $i, \overline{\left|\Delta p_{i}\right|}$, resulting in the same time scaling for the same seasonal period $i, i=1, \ldots, N$. Hence, this $\vartheta$-time scaling filtering procedure belongs to the deterministic filtering process category. In contrast to Andersen and Bollerslev (1997a) and Engle, et al. (2006), the daily volatility component is not preserved in this $\vartheta$-time scaling filtering procedure, since the daily volatility is not adjusted as a predetermined value before the average of the price change at the physical time interval $i, \overline{\left|\Delta p_{i}\right|}$, is computed. Hence, the $\vartheta$-time scaling filtering procedure inject statistical noise into both the estimated seasonal volatility and the volatility of the filtered returns because the intraday seasonal volatility will include the interday volatility component.

Andersen and Bollerslev (1997b) and Gençay, Selçuk and Whitcher (2001) employ a low-pass filtering technique in an attempt to capture all of the volatility components that are higher than a one-day frequency. Their low-pass filter is based on the Fourier transform technique and the multi-resolution analysis (MRA) of discrete wavelet transforms (DWT), respectively. Application of the low-pass filter to high-frequency data removes all of the variations at the intraday frequencies, but includes the variations at low interday frequencies. In this way, the intraday seasonal volatility is completely removed

\footnotetext{
${ }^{10}$ See Dacorogna, et al. (1993) Table 1.3, p. 424 for the detailed values of these constants.
} 
from the time series. However, the intraday conditional volatility is also removed as a tradeoff of removing the intraday seasonality. ${ }^{11}$

Beltratti and Morana (2001) propose a stochastic volatility model which captures both the deterministic and stochastic seasonal components. Results show that their stochastic volatility model performs better than the FFF approach (Andersen and Bollerslev, 1997a) and the $\vartheta$-time scaling approach (Dacorogona, et al., 1993). However, the dynamics of stochastic seasonal component still deterministic by using the FFF approach, and the estimation procedure is complex. ${ }^{12}$ Omrane and Bodt (2007) use the method of self-organizing neural network learning and nonlinear discrete projection to

${ }^{11}$ MRA is an analysis technique used to decompose a time series into many subset series disaggregated by scale (frequency). The advantage of the DWT technique over the discrete Fourier transform (DFT) is that DWT can capture localized events, whereas the DFT cannot capture such events. This is because the Fourier transform is parametric, while the transform in wavelet analysis is a nonparametric operation for local observations. The parametric estimation method of the DFT, as given below, does not effectively capture localized events:

$$
Y_{t}=A_{0}+\sum_{j=1}^{M}\left\{A_{j} \cos \left(\omega_{j} t\right)+B_{j} \sin \left(\omega_{j} t\right)\right\}+\varepsilon_{t}, M \leq(T-1) / 2
$$

where OLS is applied to the above equation for frequency $\omega_{j}=2 \pi j / T, j=1,2, \ldots,(\mathrm{T}-1) / 2$ where $\mathrm{T}$ is odd, to obtain the coefficients. Information arrivals of the frequency $\omega_{i}$ are reflected into the magnitude and significance of the coefficients. Now, suppose there is a new information arrival which causes a localized movement in returns over a given time period. Because the above OLS coefficients will be economically zero and insignificant for the localized event, the localized information arrival is not captured by the DFT method. Unlike the DFT, the DWT captures localized events because it uses a non-parametric approach without any pre-specified model. The DWT, which is a series of algebraic operations, is performed at first with a small time scale (for example, 10-minutes) separately for 'local' observations (see Jensen and CourHarbo, 2001, chapters 3, 4, and 5). If the wavelet transform coefficients (which are functions of frequency), are inversely transformed then the resulting series is a high pass filtered time series. Every information arrival with a 10-minute or less time scale is captured by the high pass filtered time series. Hence, there is no information loss by using the DWT method. The wavelet transform can be applied with larger time scales, for example, 20-minute, 40-minute, etc.

12 As Beltratti and Morana (2001) note, "We model $c(i, t, n)$ [stochastic variance component] the fundamental daily frequency, as stochastic while its harmonics are modeled as deterministic as for Andersen and Bollerslev (1997)" on page 208. The maximum likelihood estimation for the stochastic volatility model can be done by only simulations. Otherwise, Kalman filtering method is used by assuming the log of squared residuals follow the normal distribution, resulting in a quasi-maximum likelihood estimation. 
capture the both the deterministic and stochastic seasonal components. However, the neural network method does not provide the dynamics of the seasonal variance. As a result, it is not possible to find out the contribution of each deterministic and stochastic component to the total seasonal variance. In addition, filtering results of the neural network filtering procedure is not perfect in that the variance and the quoting activity of the filtered returns still exhibit seasonal movement. ${ }^{13}$

In this study I propose a different approach to filtering the intraday seasonal volatility pattern. This new approach filters out the time-varying seasonal volatility pattern, without also removing the intraday conditional volatility, and the estimation procedure is simple. This method also overcomes the problem of impounding new noise into the filtered series.

\subsection{Modeling the dynamics of intraday seasonal volatility}

I model the dynamics of the intraday seasonal volatility pattern in order to capture the time-varying aspects of this pattern. The dynamics for the intraday seasonal volatility should capture changes in the seasonal pattern as well as the persistency of the pattern. I propose a GARCH-type autoregressive filtering process, with the assumption that the dynamics of seasonal volatility of each intraday period follows a distinct autoregressive moving average process (ARMA).

Cleveland and Tiao (1979) and Vecchia (1985) propose a periodic autoregressive moving average (PARMA) model when the data has a periodic characteristic in the

\footnotetext{
${ }^{13}$ See Figure 3 in Omrane and Bodt (2007). The autocorrelation functions of the volatility and quoting activity deseasonalized by the neural network method still exhibit the periodic fluctuations.
} 
mean. ${ }^{14}$ They recognize that each element of the seasonal pattern has a unique seasonal mean process since economic agents behave differently in different seasons. Specifically, they propose the unique ARMA process for each season $(n)$ :

$\phi^{(i)}(B)\left(R_{t \mid n}-\mu_{n}\right)=\theta^{(j)}(B) r_{t \mid n}$, for $n=1, \cdots, N$

$\phi^{(i)}(B)$ and $\theta^{(j)}(B)$ are the parameter polynomials, $t \mid n$ represents time $t$ in season $n$, $r_{t, n} \sim$ iid $N\left(0, \sigma_{n}^{2}\right)$ are residual returns, $\mu_{n}$ and $\sigma_{n}^{2}$ are the mean and the variance for each element of the seasonal pattern in season $n$. However, their model does not consider the seasonal variance process for $\sigma_{n}^{2}$ which includes both the seasonal variance component $\left(s_{t \mid n}\right)$ and non-seasonal variance components.

I follow the idea in Cleveland and Tiao (1979) and Vecchia (1985) to capture the seasonality in variance in that each element $\left(s_{t \mid n}\right)$ of the seasonal variance pattern in season $n$ has a unique ARMA process. Following Andersen and Bollerslev (1997a), the total conditional variance is expressed as a multiplicative product of the daily variance and the intraday seasonal variance components for the filtering process. Specifically, the autoregressive conditional seasonal variance (ARCSV (q,p)) filtering model is stated as follows: ${ }^{15}$

\footnotetext{
${ }^{14}$ See also Tiao and Grupe (1980). They quantify the loss of prediction efficiency of the standard ARMA model, which does not adjust the seasonality relative to their PARMA models.

${ }^{15}$ I perform $N$ separate estimations, since the filtering process does not attempt to capture the intraday conditional heteroskedasticity as done in Dacorogna, et al. (1993) and Andersen and Bollerselv (1997a). Therefore, as Andersen and Bollerselv (1997a) note, the filtering process serves to eliminate the periodic components prior to the analysis of any intraday return volatility dynamics left in filtered returns. See Appendix 1.1 for the details on the consistency and the asymptotic normality of the parameters for the model in (3) and (4). In addition, this study does not attempt to filter any seasonality other than the daily Ushape seasonality as done in Andersen and Bollerslev (1997a) and Engle, et al. (2006). Especially, Daal, Farhat, and Wei (2006) provide the empirical evidence that the maturity effect is absent in the majority of
} 


\section{Mean model:}

$\phi^{(i)}(B)\left(R_{\tau}-E\left[R_{\tau}\right]\right)=\theta^{(j)}(B) r_{\tau}, \tau=1, \cdots, T N$

ARCSV model:

$r_{t \mid n}=N^{-0.5} \sqrt{\hat{h}_{t} S_{t \mid n}} V_{t \mid n}$, for each of $n=1, \cdots, N$,

$S_{t \mid n}=\alpha_{0 \mid n}+\alpha_{1 \mid n}^{(q)}(B) \tilde{\varepsilon}_{t \mid n}^{2}+\beta_{1 \mid n}^{(p)}(B) s_{t \mid n}$

where $R_{t \mid n}$ are raw returns, $r_{t \mid n}$ are the residual returns in season $n, h_{t}$ is the daily variance component, $v_{t \mid n}$ is white noise in season $n$ and asymptotically follows iid $N(0,1)$, $S_{t \mid n}$ is the intraday seasonal variance component in season $n, \tilde{\varepsilon}_{t \mid n}=r_{t \mid n} / \sqrt{\hat{h}_{t}}, t$ represents the day, $t=0, \cdots, T-1$, and $n$ is the intraday period, $n=1, \cdots, N$. Each residual process $r_{t \mid n}$ is covariance stationary. ${ }^{16}$ The set $\left\{s_{t \mid 1}, s_{t \mid 2}, \cdots, s_{t \mid N}\right\}$ represents the pattern of the intraday seasonal variance component for day $t$, each element $s_{t \mid n}$ of which is assumed to follow a distinct seasonality process, as shown in (4). The daily variance $\left(h_{t}\right)$ can be obtained by a relevant daily variance model, such as a daily MA(1)-GARCH(1,1) model (Andersen and Bollerslev, 1997a), daily commercially available variance forecasts (Engle, et al., 2006), or daily realized variance (Andersen, Bollerslev, Diebold and Labys, 2003;

Engle and Gallo, 2005). In this study, the daily realized variance, defined as $h_{t}=\sum_{n=1}^{N} r_{t n}^{2}$, is

futures contracts, where the maturity effect represent increases in the volatility of future prices near the maturity dates.

${ }^{16}$ See Appendix 1.2 for covariance stationarity of the residual process $\left(r_{t}\right)$. 
used. ${ }^{17}$ The model in (2), (3) and (4) are estimated by two-step estimation approach. As Greene (2000, p. 134) note, two-step estimators provide a simpler alternative to complicated joint estimators. In the first step, the parameters of the mean model in (2) and the daily variance component $\left(h_{t}\right)$ are estimated. The parameters of the variance filtering model in (3) and (4) are determined in the second step. ${ }^{18}$

Note that each ARCSV process $s_{t \mid n}$ involves its own parameters $\left\{\alpha_{0 \mid n}, \alpha_{1 \mid n}^{(q)}, \beta_{1 \mid n}^{(p)}\right\}$.

The parameter $\alpha_{0 \mid n}$ captures the deterministic component in $s_{t \mid n}$ (the unconditional mean of $\left.s_{t \mid n}\right), \alpha_{1 \mid n}^{(q)}$ captures innovations in $s_{t \mid n}$, while $\beta_{1 \mid n}^{(p)}$ captures the persistency in $s_{t \mid n}$. Because $s_{t \mid n}$ is forecasted by itself $\left\{s_{t-1 \mid n}, \cdots, s_{t-p \mid n}\right\}$, the innovations $\left\{\tilde{\varepsilon}_{t-1 \mid n}^{2}, \cdots, \tilde{\varepsilon}_{t-q \mid n}^{2}\right\}$ only at seasonal lags and the unconditional mean $\left(\alpha_{0 \mid n}\right)$, the forecasted values $\left(s_{t, n}\right)$ reflect only the daily periodic component of the variance, being independent of the intraday conditional heteroskedasticity. Therefore, the innovation (the seasonality innovation) captured by the ARCSV process at period $\{t, n\}$ is only the seasonality part of the total innovation for this period $\{t, n\}$. The remaining part of the total innovation at $\{t, n\}$ reflects the innovation that is forecastable by an intraday conditional heteroskedasticity process. This characteristic of the ARCSV process is rewritten as a Theorem.

\footnotetext{
${ }^{17}$ Note that the ARCSV filtering model does not restrict the daily variance model.

${ }^{18}$ Providing that the mean model in a two-step estimation is correctly specified, as Engle and Sheppard (2001) point out, the standard errors of the variance filtering model are not affected by the parameters of the mean model because the expected cross partial derivatives of the log-likelihood function with respect to the mean and the seasonal variance parameters are zero when using the normal likelihood (See Greene (2000), p. 108 and 131). A maximum likelihood estimation procedure is employed to determine the parameters in (4). See also Engle and Sheppard (2001), Engle (2002), and Engle, Sokalska and Chanda (2006) for the application of the two-step estimation and the zero-mean specification for their variance models.
} 
Theorem 1. The autoregressive conditional seasonal variance (ARCSV $(p, q))$ filtering process captures only the daily periodic variance component at the seasonal frequencies.

Proof: See Appendix 1.3.

\subsection{Gains in filtering performance of ARCSV process}

There are two major reasons why the ARCSV model is better than the extant deterministic filtering models:

a. The ARCSV filtering model improve the filtering performance by capturing the innovation in the seasonal variance process.

b. The ARCSV filtering model does not create any statistical noise both in the filtered variance series and in the filtered non-seasonal variance series.

This section elaborate on the item a. The item $b$ is examined in the section 6 . The existing deterministic filtering models, such as the FFF model (Andersen and Bollerslev, 1997a) and the variance mean filtering model (Andersen, et al., 2003; Engle et, al., 2006), captures the unconditional mean $\left(E\left[s_{t \mid n}\right]\right)$ of $s_{t \mid n}$, resulting in the seasonal variances $E\left[S_{t}\right]=\left\{E\left[S_{t \mid 1}\right], \cdots, E\left[S_{t \mid N}\right]\right\}$ for $t=0, \cdots, T-1$. Hence, the intraday seasonal variance component is explained by only its unconditional mean for each $n$. It is shown in Theorem 2 that the unconditional mean $\left(E\left[s_{t \mid n}\right]\right)$ of the intraday seasonal variance component for each of intraday period $n$ is captured by $\alpha_{0 \mid n}$ in the ARCSV filtering model. However, if the seasonal variance component $\left(s_{t \mid n}\right)$ is time-varying, then the pure deterministic filtering models lose its performance in filtering the seasonal variance 
component since they do not capture the time-varying component of the seasonal variance. The ARCSV filtering process gains its performance in filtering the seasonal variance component by capturing the unconditional mean, persistency and innovation of the seasonal variance component. Hence, the increase in filtering performance of ARCSV model relative to the pure deterministic filtering models will be positively related to the size of $\alpha_{1 \mid n}^{(q)}$ and $\beta_{1 \mid n}^{(p)}$. Theorem 3 quantifies the gain in filtering performance of ARCSV model relative to the pure deterministic filtering models.

Theorem 2. The pure deterministic seasonal variance filtering models, such as the flexible Fourier form approach (Andersen and Bollerslev (1997a) and the variance mean filtering model (Andersen, Bollerslev, Diebold and Labys, 2003; Engle, Sokalska and Chanda, 2006), are special cases of the $\operatorname{ARCSV}(q, p)$ filtering model.

Proof: see Appendix 1.4.

Theorem 3. Gain in filtering performance of ARCSV model relative to the pure deterministic filtering models is $N^{-1} \sum_{n=1}^{N}\left(\sum_{i=1}^{q} \alpha_{1 \mid n-i}+\sum_{j=1}^{p} \beta_{1 \mid n-j}\right)$ in percentage value.

Proof: See Appendix 1.5.

\subsection{Testing for ARCSV}

Whether the gain in filtering performance of $\operatorname{ARCSV}(q, p)$ model relative to the pure deterministic filtering models is statistically significant or not is an empirical matter. 
Testing the statistical significance of the gain in performance of ARCSV model relative to the pure deterministic filtering models is equivalent of testing the statistical significance of using the $\operatorname{ARCSV}(q, p)$ model. In addition, a significant stochastic seasonal variance component indicates the maladjustment for seasonality by deterministic filtering models. If a seasonal variance series can be explained by only the unconditional mean, then the following null hypothesis will be accepted:

$H_{0}: \alpha_{1 \mid n-1}=\cdots=\alpha_{1 \mid n-q_{n}}=\beta_{1 \mid n-1}=\cdots=\beta_{1 \mid n-p_{n}}=0$ for all $n=1, \cdots, N$

Since the variance model in (4) is a univariate $\operatorname{GARCH}(\mathrm{q}, \mathrm{p})$ model, the Lagrange multiplier (LM) test can be applied as shown in Engle (1982) and Bollerslev (1986). The test procedure is to run the following OLS regression and to obtain the coefficienst of regression (R-squares).

$\tilde{\varepsilon}_{t \mid n}^{2}=a_{0}+a_{1 \mid n} \tilde{\varepsilon}_{t \mid n-1}^{2}+\cdots+a_{1 \mid n-q_{n}} \tilde{\varepsilon}_{t \mid n-q_{n}}^{2}+u_{t \mid n}$ for $n=1, \cdots, N$

Let $R_{n}^{2}$ be the R-square of the intraday period $n$ from the OLS regression in (6). There will be $\mathrm{N}$ R-squares from $\mathrm{N}$ regressions because there are $\mathrm{N}$ intraday periods, and its intraday period has its seasonal variance process as shown in (4). Since $T \cdot R_{n}^{2}$ follows $\chi^{2}\left(q_{n}\right)$ in the null hypothesis, the test statistic for the null hypothesis given in (5) is

$$
\begin{aligned}
& \sum_{n=1}^{N} T \cdot R_{n}^{2} \text { which follows } \chi^{2}\left(\sum_{n=1}^{N} q_{n}\right) \text { in the null hypothesis. } \\
& \sum_{n=1}^{N} T \cdot R_{n}^{2} \sim \chi^{2}\left(\sum_{n=1}^{N} q_{n}\right)
\end{aligned}
$$




\subsection{Testing for specification and model selection}

Nerlove (1964) points out that the procedure to remove seasonality as employed by the Bureau of Labor Statistics (BLS) removes far more variation from the applicable series than can properly be considered as seasonal. Andersen and Bollerslev (1997a) point out that their FFF model for seasonality filtering injects additional noise or a bias into the filtered return series of the S\&P 500 index futures for years 1986 to 1989 , as shown by the correlogram of their absolute filtered returns being positioned substantially above that of the original absolute return series in their figure 7(b), and the correlogram also possesses a remaining periodic pattern as well. Hence, it is indispensable to perform specification tests for the seasonality filtering process to check whether any statistical distortions are made or not. Correlogram and cross-spectral tests can be used to assess the performance of the autoregressive filtering process, which is done in the remainder of this section. Additionally, the performance of the autoregressive filtering process is contrasted to that of the FFF model and the variance mean filtering model. The absolute values of the unfiltered and filtered returns are employed as measures of volatility for the correlogram and cross-spectral analysis. ${ }^{19}$

Section 6.1 examines whether GARCH-type autoregressive volatility modeling for the filtering process provides an acceptable model. Sections 6.2 and 6.3 access the performance of the autoregressive filtering process by employing the autocorrelogram and cross-spectral analysis. Section 6.4 compares the performance of the filtering models in terms of their ability to fit the seasonal variance series.

\footnotetext{
${ }^{19}$ For the following specification tests, the unfiltered absolute returns and filtered absolute returns are defined as follows: Unfiltered absolute returns $=\left|r_{\tau}\right|$ and filtered absolute returns $=\left|r_{\tau} / \sqrt{\hat{s}_{\tau}}\right|$.
} 


\subsubsection{Correlogram tests of the seasonal volatility at seasonal lags}

The ARCSV process in (4) employs an ARCH term and a GARCH term as a part of the process. We must check whether using a GARCH procedure creates any new potential problems. The reason why using GARCH modeling can be a hazardous undertaking in the presence of intraday seasonal volatility is because GARCH models only accommodate a geometric decay and cannot properly adjust the seasonal fluctuation of the autocorrelogram of the conditional (seasonal) volatility. If the actual filtering model correlogram given in equation (8) shows a geometric decay pattern, then the intraday seasonal volatility in (4) from a sample time series also will not have any seasonal fluctuation in its correlogram. In order to check for periodicity in the intraday seasonal volatility series $\sqrt{s_{t, n}}$ at the seasonal lags, I estimate the following correlogram using normalized absolute returns with seasonal lags $k$ for each point of time $\{t, n\}$.

$$
\rho_{t, n}(k)=\operatorname{corr}\left(\left|\frac{r_{t, n}}{\sqrt{\hat{h}}}\right|,\left|\frac{r_{t+k, n}}{\sqrt{\hat{h}_{t+k}}}\right|\right) \text { for } t=1, \cdots, T, n=1, \cdots, N \text { and } k=1, \cdots, T-1 .
$$

This correlogram is examined to determine whether a monotonic geometric decay pattern exists at the seasonal lags $k$.

\subsubsection{Correlogram tests for the performance of the ARCSV process}

When a combination of intraday seasonal volatility, intraday conditional nonseasonal volatility, and interday conditional volatility exists, then the correlogram of the unfiltered absolute intraday returns exhibits a regular fluctuation on a seasonal basis with a one day period, as well as a slow geometric decay in the average level of the 
correlations. ${ }^{20}$ Hence, after the removal of the intraday seasonal component, the autocorrelogram of the filtered absolute returns reflects only the non-seasonal volatility with a geometric decay pattern. Therefore, when one applies a successful deseasonalizing model to a time series that possesses a regular daily seasonal volatility pattern then the correlogram of the original absolute returns will exhibit a regular seasonal fluctuation, whereas the correlogram of the filtered absolute returns does not show any seasonality (see Figure 1.1). The filtering process smoothes the correlogram of the absolute unfiltered return series, removing the seasonal fluctuations, with the correlogram of the resulting absolute filtered returns being positioned near the mean of the absolute unfiltered returns cyclical pattern. ${ }^{21}$ If the correlogram of the filtered absolute returns retains a regular seasonal fluctuation or is positioned well above or below the correlogram of the original absolute returns due to additional noise, then a maladjustment of the filtering process has occurred. The correlogram of the absolute filtered returns is measured as follows: $:^{22}$

\footnotetext{
${ }^{20}$ The correlogram of the total filtered conditional volatility after removal of the seasonal component does not exhibit any regular seasonal fluctuation, and the correlogram only shows a geometric decay if the volatility process has persistency but no seasonality. Since the intraday non-seasonal volatility is less persistent relative to the interday volatility, the autocorrelogram of the intraday non-seasonal volatility will be positioned below the interday volatility autocorrelogram, with a geometric decay pattern. See Andersen and Bollerslev (1997a, p. 125-129) for a detailed discussion on the correlogram of absolute returns with an intraday periodic volatility component.

${ }^{21}$ This occurs due to the behavior of the intraday seasonal variance component $s_{t, n}$. The correlogram of $s_{t, n}$ or $\operatorname{corr}\left(s_{i, n}, s_{j, m}\right)$ starts with positive values in the beginning, turns to negative values in the middle, and returns to positive values as the lag length approaches a complete one day seasonal lag. See Figure 5 in the results section as well as Andersen and Bollerslev (1997a, p. 128).

${ }^{22}$ The correlogram in (6) differs from the one in (5). Equation (6) measures the correlogram with the intraday frequency lags while equation (5) measures the correlogram with the seasonal frequency lags (the lags of the integer multiples of one day).
} 
$\rho_{\tau}(m)=\operatorname{corr}\left(\left|\frac{r_{\tau}}{\sqrt{\hat{s}_{\tau}}}\right|,\left|\frac{r_{\tau+m}}{\sqrt{\hat{s}_{\tau+m}}}\right|\right)$ for $\tau=1, \cdots, T \cdot N$ and $m=1, \cdots, T \cdot N-1$.

where $\tau$ represents the cumulative intraday index that ranges from 1 to $T \cdot N$.

\subsubsection{Cross-spectral tests}

When a time series is composed of trend, seasonal, and noise components, the spectral density of the unfiltered (original) series reflects all three components. ${ }^{23}$ The trend component occurs over a long period of time and therefore contributes to increases in the spectrum at low frequencies. The seasonal component shows up as peaks in the spectrum at the seasonal frequencies. If the series is composed of only noise, then the spectrum will be flat over the entire range of frequencies.

Nerlove (1964) states that seasonality can be defined as a characteristic of a time series that gives rise to spectral peaks at seasonal frequencies. ${ }^{24}$ There are 81,47 , and 288

${ }^{23}$ The periodogram is the variance of the time series at a specific frequency. The periodogram (more precisely, the periodogram divided by $4 \pi$, see Fuller (1995) p. 359) is an estimator of the spectral density; but it is not a consistent estimator because its variance does not approach zero as the sample size grows. To overcome this inconsistency problem, 'smoothing' is applied to the periodogram by replacing the periodogram by a weighted average of the periodograms at neighboring frequencies. The number of neighboring frequencies to be included is determined by the width of the window, which is called the 'bandwidth.' The bandwidth needs to be large enough to insure the consistency of the spectral density and other cross-spectral quantities, such as the coherence and the phase spectrum. However, widening the bandwidth too much causes a distortion of the spectral density at neighboring frequencies within the bandwidth, namely causing a 'leakage' problem. If there are very large variances at some frequencies then the large variance can be leaked into neighboring frequencies through the weighted average. Hence, widening the bandwidth creates a tradeoff between consistency gain and leakage. The determination of the bandwidth depends on the researcher's judgment. (See Nerlove (1964) regarding the leakage problem.) In this study, spectral density refers to the 'smoothed' periodogram. Spectral density, spectrum, and power spectrum are interchangeably used in this study (these concepts are described in Fuller, 1995, chapter 7). Here a rectangular weighting scheme with a bandwidth of 15 is applied to the unfiltered and filtered series for the spectral analysis. There was no difference in results from using other bandwidths. The reason why the rectangular weighing function is used is because this process provides simpler testing procedures for the coherence and the phase spectrum.

${ }^{24}$ See Appendix 1.3 for the definition of seasonal frequency. 
five-minute within-day periods, i.e., $N=81,47$, and 288 for the S\&P 500 index futures, live cattle futures, and the spot exchange rate of the Japanese yen to the U.S. dollar (JPYUSD), respectively. Hence, the first intraday three major seasonal periods are at $n$ of 81 , 40.5, and 27 for the S\&P 500 futures; 47, 23.5, and 15.67 for the live cattle futures; and 288, 144, and 96 for the JPY-USD rates, respectively. (see Figure 1.2).

\section{Spectral density}

The estimated seasonal variance series needs to possess non-zero spectral density only at the specified seasonal frequencies. If the estimated seasonal variance series possesses non-zero spectral density at the non-seasonal frequencies, then the filtering process is inappropriately adding or reducing the variance at those non-seasonal frequencies, resulting in a statistical distortion of the filtered time series.

If the spectral densities of an unfiltered and a filtered absolute return series are compared, then peaks exist for the spectral density in the unfiltered series at the seasonal frequencies, but these peaks are removed in the associated filtered series. Hence, the spectral densities of a filtered series at the seasonal frequencies will be almost equivalent to the spectral densities of the neighboring non-seasonal frequencies. If the spectral densities continue to have peaks for the seasonal frequencies in the filtered series after filtering, then this implies a maladjustment of the filtering process. Moreover, the filtering process should neither add nor remove any variation at the non-seasonal frequencies. If it does then the spectral density of the filtered series will be positioned above or below that of the unfiltered series for the non-seasonal frequencies. If changes in the spectral density of the filtered series at the non-seasonal frequencies occur, then this also represents a maladjustment of the filtering process. 


\section{Coherence}

Coherence is a measure of the correlation between two series as a function of the frequency. If the coherences of the unfiltered and filtered absolute return series are compared then the coherence should be low at the seasonal frequencies because the variances at the seasonal frequencies should no longer exist for the filtered series, while they are present in the unfiltered series. Hence, the coherences enable us to test whether the filtering process injects statistical noise into the filtered time series. If the coherences between the unfiltered and filtered series are indistinguishable from 1.0 at the seasonal frequencies, then a maladjustment of the deseasonality procedure exists. ${ }^{25}$ Because the variances at the non-seasonal frequencies should not be removed by the filtering process, the coherence of the unfiltered and filtered series should be 1.0 at these non-seasonal frequencies. If the coherence is statistically different from 1.0 at these frequencies then the filtering process has inappropriately removed variances of the series at the nonseasonal frequencies. ${ }^{26}$

Phase

The phase statistic estimates the phase shift between two series, that is, the extent by which one series leads or lags another series. ${ }^{27}$ If there is a nonzero phase then any statistical causal relation between any two filtered series will be misleading. Hence, the phase between the unfiltered and filtered absolute return series should not be significantly

\footnotetext{
${ }^{25}$ The coherence of two identical time series is always 1.0 at all frequencies.

${ }^{26}$ Since it is not possible to test directly the null hypothesis that coherence equals 1 , I test the null hypothesis that coherence equals 0.99. The test procedure for the coherence is described in Appendix 1.3.

${ }^{27}$ The phase spectrum of two identical time series is always zero at all frequencies.
} 
different from zero. The testing procedure for the phase is explained in Fuller (1995, p. 392-393) and Brockwell and Davis (1991, p. 449).

\subsubsection{Model selection}

This section compares the performance of the variance seasonality filtering models in terms of their ability to fit the seasonal variance series. The filtering models examined are the FFF model, the variance mean filtering model, and the ARCSV model. The timescaling procedure (Dacorogna, et al., 1993) is not included in this comparison because this procedure does not adjust the daily variance component separately, as discussed in the literature review.

Appendix 4 shows that the seasonal variance estimated by both the variance mean filtering model and the FFF model (without the interaction terms) are special cases of the ARCSV process developed in this paper. If the ARMA formulation of the ARCSV process is a better approach to capture the intraday seasonal variance then the ARCSV model will result in a better model selection. The procedure to examine in-sample fit is accomplished by employing the following regressions. ${ }^{28}$

$\log \left(r_{t, n}^{2}\right)=a_{0}+a_{1} \log \left(\hat{h}_{t} \hat{s}_{t, n}^{j}\right)+u_{t, n}$ for $j=\{A R C S V, F F F$, Mean $\}$

$\log \left(r_{t, n}^{2}\right)=b_{0}+b_{1} \log \left(\hat{h}_{t} \hat{s}_{t, n}^{A R C S V}\right)+b_{2} \log \left(\hat{h}_{t} \hat{s}_{t, n}^{F F F}\right)+u_{t, n}$

\footnotetext{
${ }^{28}$ My ARCSV filtering model, the FFF model, and the mean filtering model have the following formulation:

$r_{t, n}=N^{-0.5} \sqrt{h_{t} s_{t, n}} v_{t, n}$, where $r_{t, n}$ is the residual returns with mean zero, and $s_{t, n}$ is described by the deterministic flexible Fourier series for the FFF model and by a constant for each $n$ for the mean filtering model while $S_{t, n}$ is estimated by ARMA(q,p) for the ARCSV filtering model. Regression equation (10) is obtained by squaring and taking the logarithm for the both sides of $r_{t, n}=N^{-0.5} \sqrt{h_{t} s_{t, n}} v_{t, n}$. Regressions in (10) through (13) are similar to those in Martens, et al. (2002), p. 291. Regressions, such as (11), (12), and (13), which compare the relative performance of forecasts are called encompassing regressions (see Greene, 2000, p. 301; Canina and Figlewski, 1993).
} 
$\log \left(r_{t, n}^{2}\right)=b_{0}+b_{1} \log \left(\hat{h}_{t} \hat{s}_{t, n}^{\text {ARCSV }}\right)+b_{2} \log \left(\hat{h}_{t} \hat{s}_{t, n}^{\text {Mean }}\right)+u_{t, n}$

$\log \left(r_{t, n}^{2}\right)=b_{0}+b_{1} \log \left(\hat{h}_{t} \hat{s}_{t, n}^{\text {Mean }}\right)+b_{2} \log \left(\hat{h}_{t} \hat{s}_{t, n}^{F F F}\right)+u_{t, n}$

where $\hat{s}_{t, n}^{A R C S V}, \hat{s}_{t, n}^{F F F}$, and $\hat{s}_{t, n}^{\text {Mean }}$ are the seasonal variances estimated by the ARCSV, FFF, and variance mean filtering models, respectively. If the ARCSV model better estimates the seasonal variance then the parameter estimates of the ARCSV model will be larger and will possess a greater statistical significance. The coefficient of determination $\left(R^{2}\right)$ of the ARCSV model also will be larger.

\subsection{Data}

S\&P 500 index futures and live cattle futures data for year 1999 and the spot exchange rate of Japanese yen to the U.S. dollar (JPY-USD) from December 2004 to September 2005 are employed for this study.

The returns of the S\&P 500 index and live cattle futures are computed as the differences between the logarithmic prices for the last recorded price entries of the nearby futures contract over consecutive five-minute intervals. The time period employed is from 9:30 (9:05) a.m. to 4:15 (1:00) p.m Eastern time for the S\&P 500 index (the live cattle) futures. The overnight return is excluded to ensure a consistent time interval. For the first 5-minute interval from 9:30 a.m. to 9:35 a.m. (9:05 a.m. to 9:10 a.m.) the price at 9:30 a.m. (9:05 a.m.) is used as the opening trade price for this interval for the S\&P 500 index (the live cattle) futures; other intervals employ close to close prices. In addition, the following days are dropped from the data: non-trading days, half trading days, and 
trading days within the week of expiration for the S\&P 500 index futures. ${ }^{29}$ If a given 5minute interval has no trades, then the price is given by a linear interpolation between time intervals to insure 81 (47) intraday intervals for each day for the S\&P 500 index (the live cattle) futures. There are 223 (232) trading days with the total observations of 18,063 $(10,904)$ for the S\&P 500 index (the live cattle) futures.

The one-minute JPY-USD spot exchange rate is obtained from Olsen and Associates. Following Andersen and Bollerslev (1997a), price is defined as the exponential of the midpoint of the logarithmic bid and ask rates at each time interval. The five-minute return is defined as the difference between these prices. Since the spot exchange rate markets are open 24 hours a day, there are 288 five-minute intervals in a day. Returns from Friday 16:00 p.m. through Sunday 16:00 p.m. Eastern time are excluded due to the lack of trading activity. ${ }^{30}$ Approximately $3.7 \%$ of 62,496 five-minute quotes are filtered out using the outlier filtering algorithm in Dacorogna, et al. (1993). Quotes that are filtered out are linearly interpolated.

As done in Andersen, Bollerslev, Diebold, and Ebens (2001) and Martens, et al. (2002), the following autoregression in (14) as the first step of the mean model shown in (2) is implemented for each data set to purge the returns of the negative autocorrelation

\footnotetext{
${ }^{29}$ Only the actual expiration day is excluded for live cattle futures, not all trading days within the expiration week. Non-trading days and half trading days are excluded for the live cattle futures, as was done for the S\&P 500 index futures.

${ }^{30}$ The beginning price used for the first return of the first day is the opening trade price of the first time interval. There are big jumps in quotes around the end of a day (the beginning of the next day for the exchange rate) which are not filtered out. Hence, the ending price for the return of the last five-minute interval is the price one minute prior to the last recorded price of the interval, i.e., the price at 23:59 p.m. For the same reason, the beginning price of the return for the first five-minute interval is the opening trade price of that interval. Times are not adjusted for daylight savings time, as done by Dacorogna, et al. (1993) and Andersen and Bollerslev (1997a); Martens, et al. (2002) do adjust for daylight savings time. Andersen and Bollerslev (1997a) exclude Friday 17:00 p.m. through Sunday 17:00 p.m. Eastern time for their deutschemark-U.S. dollar exchange rate data.
} 
induced by the inherent bid-ask spread, and the residuals are taken as returns $\left(r_{\tau}\right)$ for the model in (3) in the second step.

$R_{\tau}=\rho R_{\tau-1}+r_{\tau}$

where the estimated coefficient $\rho$ captures market microstructure effects, which is expected to have a negative value. The estimated $\rho$ is -0.077 for the S\&P 500 index futures, 0.0014 for live cattle futures, and -0.049 for the JPY-USD spot exchange rate.

\subsection{Results}

This section discusses whether the GARCH-type volatility modeling for the filtering process is acceptable, the estimation results of the ARCSV model, and testing the performance and validity of the ARCSV process. The autocorrelogram and crossspectral analysis are employed to examine the validity of the model. Results of the comparison of the performance of the variance seasonality filtering models are also presented.

\subsubsection{Correlogram test results of the seasonal volatility at seasonal lags}

In order to use the GARCH-type autoregressive process presented in the methodology section to model the intraday seasonal volatility, the correlogram of the intraday seasonal volatility series $s_{t, n}$ at the seasonal lags needs to show a geometric decay pattern without any regular fluctuations. The correlogram is obtained from the formula given by equation (8). Figure 1.3 shows that the estimated correlograms (the estimated correlations of the seasonal variance at the seasonal lags) of each data set for 
100 lags have a slow (geometric) decay pattern without any regular fluctuations in the decay pattern. The slow geometric decay patterns of the correlations in Figure 1.3 prove that an GARCH-type autoregressive model to estimate the intraday seasonal volatility in the proposed filtering process does not cause any bias or instability in its parameter estimates. Hence, it is an appropriate filtering process.

\subsubsection{Estimation results of the ARCSV models}

Tables 1.1 provide test results of the significance of stochastic seasonal variance component and the estimation results for the ARCSV model. Based on the R-squares obtained from the equation in (6), the test statistics are computed as shown by (7). The orders of lag for equation (6) are determined by the backward selection method starting from the maximum lag of four for each ARCSV process in season $n, n=1, \cdots, N$. Table 1.1 shows that there are significant stochastic component in the intraday seasonal variance. There are 22, 18 and 83 seasons out of the total of 47, 81 and 288 seasons, which have a statistically significant stochastic seasonal variance component for SNP 500, Live cattle futures and JPYUSD spot exchange rate. Results also show that stochastic variance component for each variance series is mostly explained by the first lag. All test statistics are significantly larger than critical values. The measures of increase in filtering performance as given by Theorem 2 show that the seasonal variance is explained by the stochastic component by $9 \%, 12.9 \%$ and $8.5 \%$ for SNP 500, Live cattle futures and JPYUSD spot exchange rate, respectively. In other words, the traditional unconditional mean (or deterministic component) account for 91\%, 87.1\% and 91.5\% for SNP 500, Live cattle futures and JPYUSD spot exchange rate, respectively. The lag orders of ARCH and GARCH terms are one $(q=p=1)$ for the estimations of the ARCSV model in 
(4). Figure 4 shows the estimated intraday seasonal volatilities $s_{t, n}$ for the first 10 days of the S\&P 500 index and the live cattle futures and the first five days of the JPY-USD exchange rate. As expected, the intraday seasonal variances are U-shaped and the Ushaped pattern changes over time. The estimation results in Table 1.2 of the FFF model with interaction terms show that the seasonal variance pattern of live cattle futures are more time-varying than the S\&P 500 futures or the JPY-USD exchange rate. The coefficients delta20 and delta21 of the interaction terms between the daily volatility and the sinusoids are significant. These significant interaction terms show that the FFF process has rescaled the size of the deterministic seasonal shape (the specified sinusoids) by the factor of the daily volatility in order to fit the time-varying seasonal pattern, raising the possibility of a maladjustment of the seasonality process. The FFF estimation results without the interaction terms exhibit significance because the multicollinearity from to the interaction terms was removed. The daily pattern of the estimated seasonal variance of in the JPY-USD is identical over the sample period because no interaction terms between the daily volatility and the sinusoids exist in the JPY-USD model.

\subsubsection{Correlogram test results of the performance of the filtering model}

Figure 1 previously showed the correlogram of the unfiltered and filtered absolute returns and Figure 5 adds the correlogram of the estimated seasonal volatility, all at the intraday lags. The correlogram of the unfiltered absolute returns in Figure 1 shows a regular seasonal fluctuation over the day and geometric decay pattern of the average level of correlations for each dataset. This pattern of the unfiltered absolute returns reflects the fact that the intraday returns are composed of the intraday seasonal variance component and the non-seasonal conditional variance component. The standard GARCH model 
cannot accommodate this regular fluctuation of the correlogram of volatility. This shows that a pure ARCH/GARCH model would provide an inaccurate intraday volatility model unless one first eliminates the intraday seasonal volatility pattern. Therefore, a wellfiltered time series has to exhibit a stable geometric decay in the correlogram of the volatility, since the filtered volatility series is composed of only the non-seasonal conditional volatility. The correlogram of the filtered absolute returns in Figure 1.1 shows a stable geometric decay pattern, which occurs because the filtered absolute return series possesses only a non-seasonal conditional volatility component. The complication created by the intraday seasonal variance component is illustrated by Figure 5. As expected (see footnote 30), in Figure 5 the autocorrelation of the seasonal volatility at the intraday lags changes its sign as the lags change. I can observe that the correlogram of the unfiltered absolute returns become smaller (larger) at the point where the correlogram of the estimated seasonal variance changes its sign from positive to negative (or negative to positive). Hence, the seasonal effect in the unfiltered series is caused by the seasonal component. Moreover, since the behavior of the seasonal component is precisely estimated and filtered out, the correlogram of the absolute filtered series is positioned near the mean of the absolute unfiltered returns, meaning that no additional noise is induced into the filtered series by the ARCSV process. The autocorrelation functions of the filtered absolute returns from the FFF model tend to lie above those of the ARCSV process for the S\&P 500 index and live cattle futures.

\subsubsection{Cross-spectral test results of the performance of the filtering model}

As previously explained, seasonality can be defined as a characteristic of a time series that causes spectral peaks at seasonal frequencies. The expectation that the major 
seasonal periods occur at the periods of $81(47,288), 40.5(23.5,144)$ and $27(15.67,96)$ for the S\&P 500 futures (live cattle futures, JPY-USD exchange rate) is verified in Figure 2 where the actual spectral densities of the estimated intraday seasonal variance series $\left(s_{t, n}\right)$ are equivalent to the expectations. Alternatively, one can look at Figure 6 and focus on the spectral density for the absolute returns for the values from 0 to 1 . In this figure the seasonal variance estimated by the ARCSV process has zero spectral density at the non-seasonal periods, meaning that the ARCSV process did not add nor did it reduce the variance of the original return series at the non-seasonal periods. However, the seasonal variance captured by the FFF model does have non-zero variance terms at the non-seasonal periods, especially for the live cattle and S\&P 500 futures contracts. Therefore, the FFF model for the futures datasets examined here introduces additional noise at the non-seasonal periods. The noise from the FFF model is more severe in the live cattle futures than in the S\&P 500 futures, while there is no noise in the JPY-USD exchange rate series. These results support the finding that the source that injects additional noise is the interaction terms, where these terms are designed to capture the time-varying seasonal pattern for the FFF model. The results in section 8.5 show that dropping out the interaction terms in order to remove the added noise injected into the series by the FFF model leads to decreased performance in fitting the seasonal variance.

Meanwhile, the spectral density of the unfiltered absolute returns for each dataset in figure 1.7 (which includes both the seasonal and non-seasonal components) exhibits peaks at the seasonal density, as expected, showing a regular and strong seasonal variance component. Figure 1.7 also shows that the peaks of the spectral density of the unfiltered absolute returns at the seasonal frequencies are removed by my ARCSV 
process, while there is no change in the spectral density at the non-seasonal frequencies. This result is consistent with a proper filtering process. In addition, Figure 1.7 shows the spectral density of the filtered absolute returns by the FFF model with the interaction terms. In accordance with the findings from the spectral density of the estimated seasonal variance, the spectral density of the filtered absolute returns from the FFF model at the non-seasonal periods lie above the unfiltered absolute returns for the S\&P 500 and below the unfiltered returns for live cattle. The changed variance at those non-seasonal periods represents the extra noise in the filtered returns arising from the FFF filtering model.

I employ two other measures (coherence and phase) from cross-spectral analysis to access the performance of the filtering processes. For a good filtering process to exist, the coherence between the unfiltered and filtered series must be low at the seasonal frequencies, while being close to one at the non-seasonal frequencies. Figure 1.8 shows the estimated coherence for each frequency and the associated 99\% confidence intervals for each dataset. The estimated coherence from the ARCSV process is smallest at the seasonal periods, in particular $81(47,288), 40.5(23.5,144), 27(15.67,96)$, etc. for the S\&P 500 index (live cattle, JPY-USD exchange rate), while it is close to one at the nonseasonal periods. The upper bounds of the $99 \%$ confidence intervals lie below the value of 0.99 at the seasonal frequencies, while they do not at the non-seasonal frequencies. These results mean that the unknown true coherence from the filtered absolute returns at the seasonal frequencies is smaller than 0.99. Therefore, the null hypothesis that the coherence is 0.99 is rejected at the seasonal frequencies, whereas it is not rejected at the non-seasonal frequencies using $1 \%$ significance levels. However, the coherence estimated by the FFF model lies below 0.99 for the S\&P 500 and live cattle in the non- 
seasonal periods, where the FFF model injected noise into the filtered time series. In particular, the noise problem is severe for live cattle. This noise problem is not present for the JPY-USD exchange rate, since the interaction terms are not included, i.e., only the unconditional mean of the seasonal variance is adjusted.

The phase statistic between the unfiltered and filtered absolute returns should not be significantly different from zero for a good filtering process. Figure 1.9 shows the estimated phase for each frequency and the associated 99\% confidence intervals. Although the 99 percent confidence bounds from the ARCSV process intersect the value of zero at some frequencies, the violations are negligible and they are point-intersections, not extensive regional intersections. The distortion in the phase relation by the FFF model is present in live cattle at the non-seasonal periods. In conclusion, the specification tests using the correlogram, spectral density, coherence, and phase procedures show that my element-wise ARCSV process for the intraday seasonal volatility performs well.

\subsubsection{Results of the in-sample fit}

Appendix 1.4 shows that the FFF model (Andersen and Bollerslev, 1997a) without interaction terms and the variance mean filtering model (Engle, et al., 2006) are special cases of the ARCSV model. Appendix 1.1 then shows that the autoregressive model will exhibit a better in-sample fit relative to these deterministic models. As expected, the regression estimation results using equation (10) in Table 1.3 show that the ARCSV model coefficients (ARCSV) and its R-squares for the seasonal variance are larger and more significant than those for the seasonal variance estimated by the FFF model (FFF) and the variance mean filtering model (Mean). In fact, these conclusions are even more prominent when one examines the estimation results from the encompassing regressions 
using equations (11) and (12). Note that the coefficients FFF and Mean for the three datasets become smaller when the seasonal variances estimated by the ARCSV model are included in the regressions. In fact, the coefficients FFF and Mean for live cattle become negative. These findings are consistent with the fact that the pure deterministic filtering models (the FFF model without the interaction terms and the variance mean filtering model) do not capture the entire seasonal variance if the seasonal pattern is sufficiently time-varying. Appendix 1.4 also shows that for a finite sample the variance mean filtering model provides better estimates for the unconditional mean of the seasonal variance $E\left[s_{t, n}\right]$ compared to the FFF model without the interaction terms. Estimation results from the regression given in (13) support the expectation that the coefficients Mean are larger in size and more significant than the coefficients FFF (for all three datasets). The results from the regressions in (13) also provide evidence that including a distinct mean for each element seasonal variance process provides better estimates for the intraday seasonal variance.

\subsection{Conclusions}

An understanding of the return and associated volatility processes of a time series is important, since such processes reflect the impact of information arrivals (Grossman, 1976; Andersen and Bollerslev, 1997b). However, the volatility due to news is difficult to separate from the intraday seasonal volatility. Therefore, the intraday seasonal volatility components must first be eliminated in order to use high frequency data to model volatility dynamics. 
Prior studies (Dacorogna, et al., 1993; Andersen and Bollerslev, 1997a; Hughes and Winters, 2005; Engle et al., 2006) assume that the intraday seasonal volatility pattern is deterministic for the process of filtering out seasonality effects. However, the intraday seasonal volatility pattern is often time-varying rather than deterministic. Alternatively, the low-pass filtering methods used with the Fourier transform (Andersen and Bollerslev, 1997b) and with the wavelet transform (Gençay, Selçuk and Whitcher, 2001) procedures eliminate the non-seasonal short-run intraday volatility component as well as the seasonality patterns. Consequently, an accurate analysis of the short-run intraday volatility behavior with these procedures is not possible. Beltratti and Morana (2001) and Omrane and Bodt (2007) recongnize the filtering procedure need to capture the stochastic seasonal component. However, Beltratti and Morana's model still requires the deterministic dynamics to decribe the stochastic seasonal component. Omrane and Bodt's model does not provide the dynamics of the seasonal variance, and the filtered series by the neural network method still exhibit seasonal behavior.

In this study I propose a different approach to modeling the intraday seasonal volatility pattern in order to overcome the problems arising from a changing seasonality pattern in combination with using a deterministic model to filter the data, as well as a model that does not remove the non-seasonal intraday conditional volatility with the seasonality factor. My model assumes that the seasonal pattern can change over time in a forecastable way, and that the intraday seasonal pattern follows an autoregressive process. These assumptions allow the model to capture both the persistency and changes in the seasonal pattern. Moreover, the estimation procedure is very simpler than prior filtering 
models. The performance of the filtering process is tested by means of a correlogram and spectral analysis.

The test results show that the new ARCSV process performs very well. In particular, there are no distortions to the harmonic properties of the filtered time series, such as in the power spectrum or phase relations at each frequency of the series. Findings in this study also show that any modification to the deterministic filtering models without including a seasonal innovation term to capture the time-varying seasonal pattern leads to statistical distortion in the resultant series. In particular, the flexible Fourier form (FFF) model injects noise into the filtered time series if the interaction terms between the daily volatility and the sinusoid terms are included to capture the time-varying seasonal pattern. Meanwhile, pure deterministic filtering models, such as the FFF model in Andersen and Bollerslev (1997a) that does not include the interaction terms and the variance mean filtering model in Engle, et al. (2006) are special cases of the ARCSV model presented in this paper (these models ignore the innovation and persistency terms). Hence, the ARMA formulation of the seasonal variance developed in this paper for each intraday interval outperforms the deterministic filtering models in capturing the seasonal variance, as shown by appendix 1 and results in this study.

In conclusion, the ARCSV process developed in this study enables one to filter out the seasonal U-shaped volatility pattern without introducing any additional noise into the filtered series. This ARCSV process is successfully applied to the S\&P 500 index futures, the live cattle futures, and the spot Japanese yen to U.S. dollar exchange rate. Hence, the ARCSV process is an appropriate tool for the analysis of high frequency data to filter out 
the time-varying intraday seasonal volatility pattern, without distorting the statistical properties of the original time series, leaving the intraday conditional volatility intact. 
Figure 1.1: Correlograms at five minute lags for the unfiltered and filtered absolute returns

Figure 1.1 graphs the autocorrelation functions of the unfiltered and the filtered absolute returns for each dataset using the ARCSV process and the FFF model.
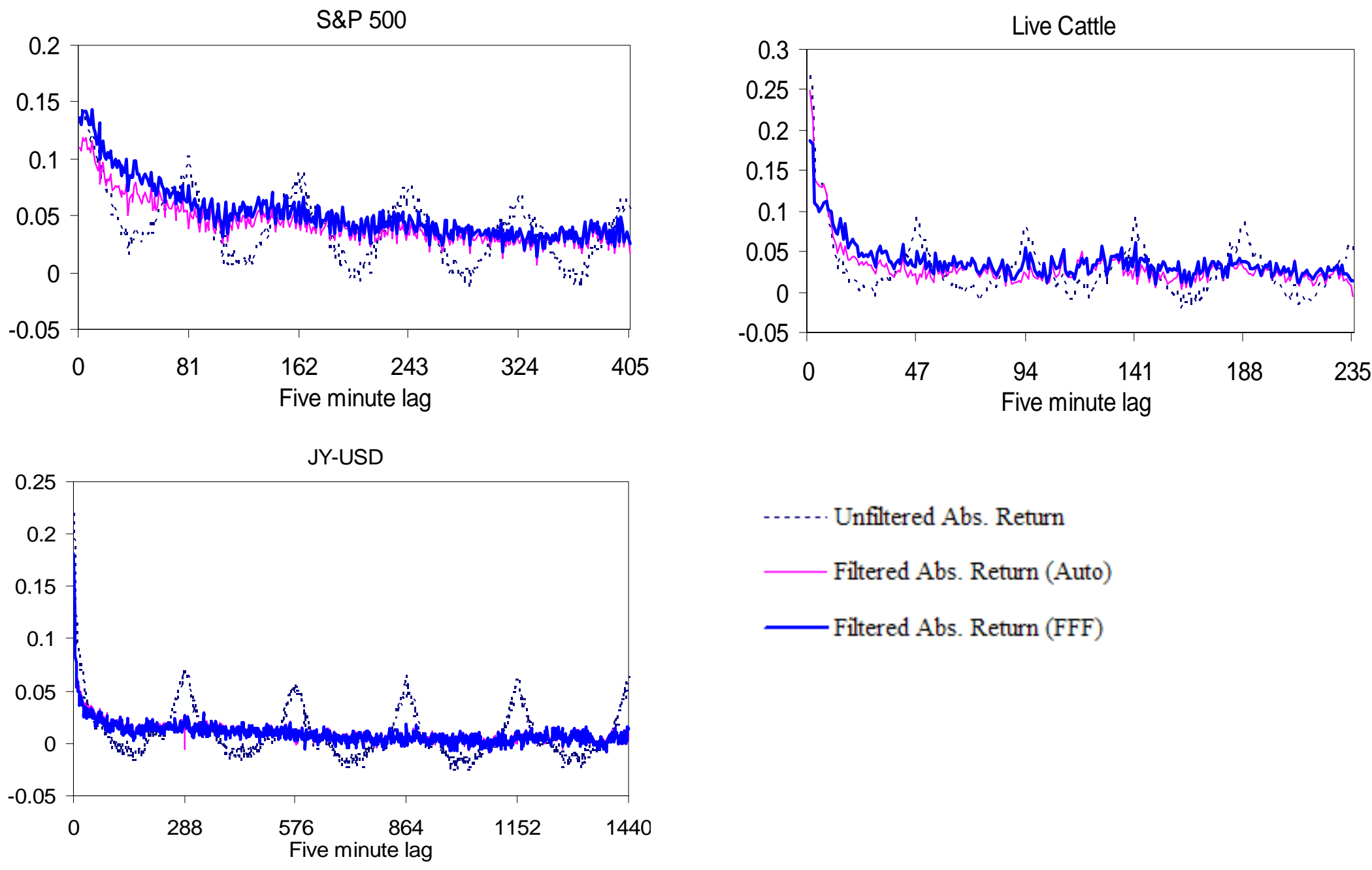
Figure 1.2: Spectral density of the intraday seasonal variance estimated by the ARCSV process

Figure 1.2 graphs the spectral density of the seasonal variance estimated by the ARCSV process. The seasonal periods that contribute to the intraday seasonal variance component are $N / n$, where $N=81,47$, and 288 for the S\&P 500 index futures, live cattle futures, and JPY-USD exchange rate, respectively. Hence, the first three major seasonal periods are 81, 40.5, and 27 for the S\&P 500 futures; 47, 23.5, and 15.67 for the live cattle futures; and 288, 144, and 96 for the JPY-USD exchange rate, respectively.

S\&P 500

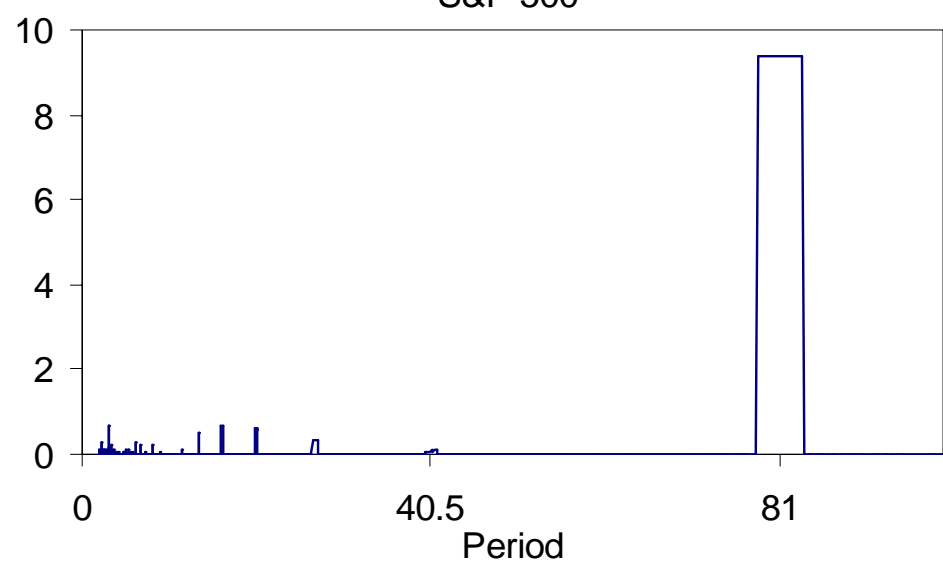

JY-USD

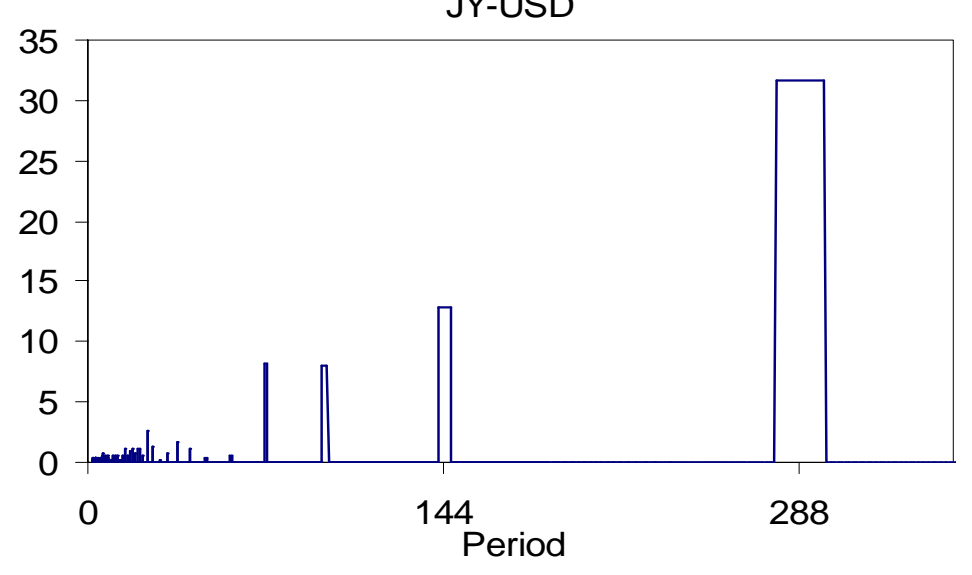

Live Cattle

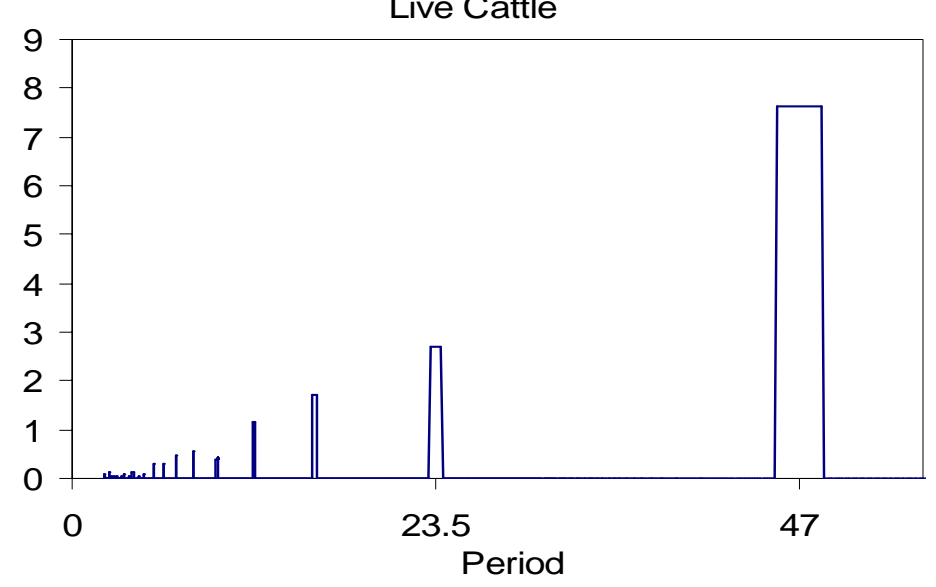


Figure 1.3: Correlogram of the normalized absolute returns at seasonal lags

Figure 1.3 graphs the estimated correlations of the normalized absolute returns for the S\&P 500 index futures, live cattle futures, and JPY-USD exchange rate for 100 seasonal lags. The correlograms in Figure 1.3 must have a slow (geometric) decay pattern without any regular fluctuations for the GARCH-type autoregressive process to be used as a filtering model.

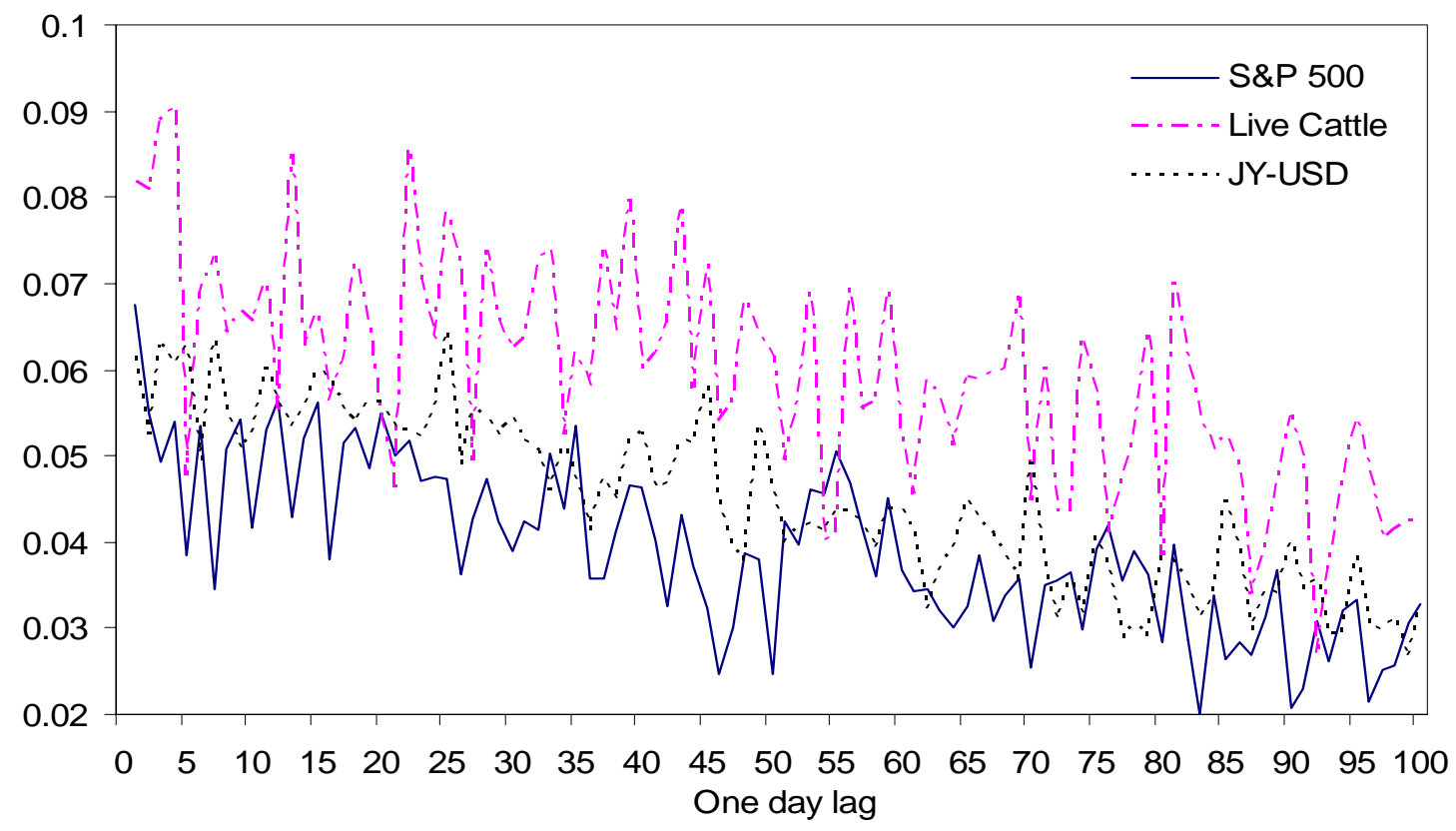




\section{Table 1.1: Estimation and Test Results of the ARCSV Process}

This table provides test results of the significance of stochastic seasonal variance component and the estimation results for the ARCSV model using 5-minute S\&P 500 index futures, live cattle futures, and the JPY-USD spot exchange rate. The tests are based on the following regression.

$\tilde{\varepsilon}_{t \mid n}^{2}=a_{0}+a_{1 \mid n} \tilde{\varepsilon}_{t \mid n-1}^{2}+\cdots+a_{1 \mid n-q_{n}} \tilde{\varepsilon}_{t \mid n-q_{n}}^{2}+u_{t \mid n}$ for $n=1, \cdots, N$.

The following test statistics are computed using R-squares obtained the above regressions.

$\sum_{n=1}^{N} T \cdot R_{n}^{2} \sim \chi^{2}\left(\sum_{n=1}^{n} q_{n}\right)$.

The ARCSV filtering model is specified as

$$
\begin{aligned}
& r_{t \mid n}=N^{-0.5} \sqrt{\hat{h}_{t} s_{t \mid n}} v_{t \mid n}, \text { for each of } n=1, \cdots, N \\
& s_{t \mid n}=\alpha_{0 \mid n}+\alpha_{1 \mid n}^{(1)}(B) \tilde{\varepsilon}_{t \mid n}^{2}+\beta_{1 \mid n}^{(1)}(B) s_{t \mid n}
\end{aligned}
$$

where $h_{t}$ is the daily variance component, $s_{t, n}$ is the intraday seasonal variance component, $t$ is the daily

\begin{tabular}{|c|c|c|c|c|c|c|c|c|}
\hline $\begin{array}{l}\text { Non-zero } \\
\text { lags / total }\end{array}$ & Lag 1 & Lag 2 & Lag 3 & $\begin{array}{l}\text { Lagrange } \\
\text { multiplier }\end{array}$ & $\begin{array}{c}\text { Critical } \\
\text { value }\end{array}$ & $\begin{array}{c}\text { Mean of } \\
\text { arch0 }\end{array}$ & $\begin{array}{c}\text { Mean of } \\
\text { arch1 }\end{array}$ & $\begin{array}{c}\text { Mean of } \\
\text { garch1 }\end{array}$ \\
\hline \multicolumn{9}{|c|}{ S\&P 500 index futures } \\
\hline $24 / 81$ & 22 & 1 & 1 & 137.72 & 35.17 & $\begin{array}{c}0.9104 \\
90.97 \%\end{array}$ & $\begin{array}{l}0.0342 \\
3.42 \%\end{array}$ & $\begin{array}{l}0.0562 \\
5.61 \%\end{array}$ \\
\hline \multicolumn{9}{|l|}{ Live cattle futures } \\
\hline $20 / 47$ & 18 & 1 & 1 & 126.84 & 40.11 & $\begin{array}{c}0.8444 \\
87.05 \%\end{array}$ & $\begin{array}{l}0.0326 \\
3.36 \%\end{array}$ & $\begin{array}{c}0.093 \\
9.58 \%\end{array}$ \\
\hline \multicolumn{9}{|c|}{ JPY-USD spot exchange rate } \\
\hline $92 / 288$ & 83 & 7 & 2 & 834.92 & 129.92 & $\begin{array}{c}0.9204 \\
91.52 \%\end{array}$ & $\begin{array}{c}0.0362 \\
3.6 \%\end{array}$ & $\begin{array}{l}0.0491 \\
4.89 \%\end{array}$ \\
\hline
\end{tabular}
index, and $n$ is the intraday index. The filtering model is estimated separately for each $n$. 


\section{Table 1.2: Estimation Results of the Flexible Fourier Form (FFF) Approach}

The following flexible Fourier form regression is implemented for each of the data sets: S\&P 500 index futures, live cattle futures, and the JPY-USD spot exchange rate:

$$
\begin{gathered}
x_{t, n}=f_{t, n}\left(\theta \mid \sigma_{t}, n\right)+u_{t, n} \\
f_{t, n}=\sum_{j=0}^{J} \sigma_{t}^{j}\left[\mu_{0 j}+\mu_{1 j} \frac{n}{N_{1}}+\mu_{2 j} \frac{n^{2}}{N_{2}}+\sum_{i=1}^{D} \lambda_{i j} I_{n=d_{i}}+\sum_{i=1}^{P}\left(\gamma_{p j} \cos \frac{2 \pi p n}{N}+\delta_{p j} \sin \frac{2 \pi p n}{N}\right)\right]
\end{gathered}
$$

where $n$ is the intraday observation interval index, $N$ is the number of intraday return intervals, $N_{1} \equiv N^{-1}(N+1)$, and $N_{2} \equiv(N+1)(N+2) / 6$, and $\sigma_{t}$ is daily volatility. Following Andersen and Bollerslev (1997a), $\mathrm{J}=1$ and $\mathrm{p}=2$ for the $S \& P 500$ index futures and live cattle futures, and $\mathrm{J}=0$ and $\mathrm{p}=6 \mathrm{for}$ the JPY-USD spot exchange rate if the FFF model includes the interaction terms. The dummies are set to one for the last three intraday time intervals $(n=79,80$, and 81$)$ for the stock index futures. The intraday seasonal variance is

\begin{tabular}{|c|c|c|c|c|c|c|}
\hline \multirow[b]{2}{*}{ Mu00 } & \multicolumn{2}{|c|}{ S\&P 500} & \multicolumn{2}{|c|}{ Live cattle } & \multicolumn{2}{|r|}{ JPY-USD } \\
\hline & 1.92 & 2.284 & 5.34 & 5.71 & $\mathrm{Mu0}$ & 3.85 \\
\hline Mu10 & 4.01 & 1.953 & -8.03 & -10.48 & Mu1 & 0.26 \\
\hline Mu20 & -1.38 & -0.669 & 2.43 & 3.43 & Mu2 & -0.09 \\
\hline Lambda10 & -0.69 & -0.568 & & & Gamma1 & -0.19 \\
\hline Lambda20 & -1.19 & -1.469 & & & Delta1 & 0.42 \\
\hline Lambda30 & 0.27 & -0.270 & & & Gamma2 & 0.06 \\
\hline Gamma10 & 1.31 & 0.920 & -1.04 & -1.48 & Delta2 & -0.23 \\
\hline Delta10 & 0.22 & -0.081 & -0.15 & -0.19 & Gamma3 & -0.29 \\
\hline Gamma20 & 0.25 & 0.110 & -0.16 & -0.28 & Delta3 & 0.13 \\
\hline Delta20 & 0.17 & 0.034 & 0.26 & -0.02 & Gamma4 & -0.20 \\
\hline Mu01 & 313.33 & & 339.68 & & Delta4 & 0.00 \\
\hline Mu11 & -1761.55 & & -2254.41 & & Gamma5 & -0.01 \\
\hline Mu21 & 610.78 & & 917.86 & & Delta5 & 0.03 \\
\hline Lambda11 & 105.33 & & & & Gamma6 & 0.03 \\
\hline Lambda21 & -230.99 & & & & Delta6 & -0.02 \\
\hline Lambda31 & -467.16 & & & & R squared & 0.035 \\
\hline Gamma11 & -333.45 & & -399.40 & & $\mathrm{~N}$ & 62,496 \\
\hline Delta11 & -265.30 & & -34.83 & & & \\
\hline Gamma21 & -123.02 & & -109.49 & & & \\
\hline Delta21 & -117.86 & & -253.44 & & & \\
\hline R squared & 0.028 & 0.025 & 0.023 & 0.022 & & \\
\hline $\mathrm{N}$ & 18,063 & 18,063 & 10,904 & 10,904 & & \\
\hline
\end{tabular}

$$
s_{t, n}=\left(\frac{T \cdot N \exp \left(0.5 f_{t, n}\right)}{\sum_{t=1}^{T} \sum_{n=1}^{N} \exp \left(0.5 f_{t, n}\right)}\right)^{2}
$$


Figure 1.4: Intraday seasonal variance estimated by the ARCSV process

Figure 1.4 plots the estimated intraday seasonal variances from the ARCSV process developed in this study. The intraday seasonal variance component has a cycle of one day. One day is composed of 81 five-minute intraday intervals for the S\&P 500 index futures, 47 intervals for the live cattle futures, and 288 intervals for JPY-USD spot exchange rate. The first 10, 10, and 5 days are shown in Figure 1.4 out of a total of 223, 232, and 217 trading days for the S\&P 500 index futures, live cattle futures, and JPY-USD spot exchange rate, respectively.
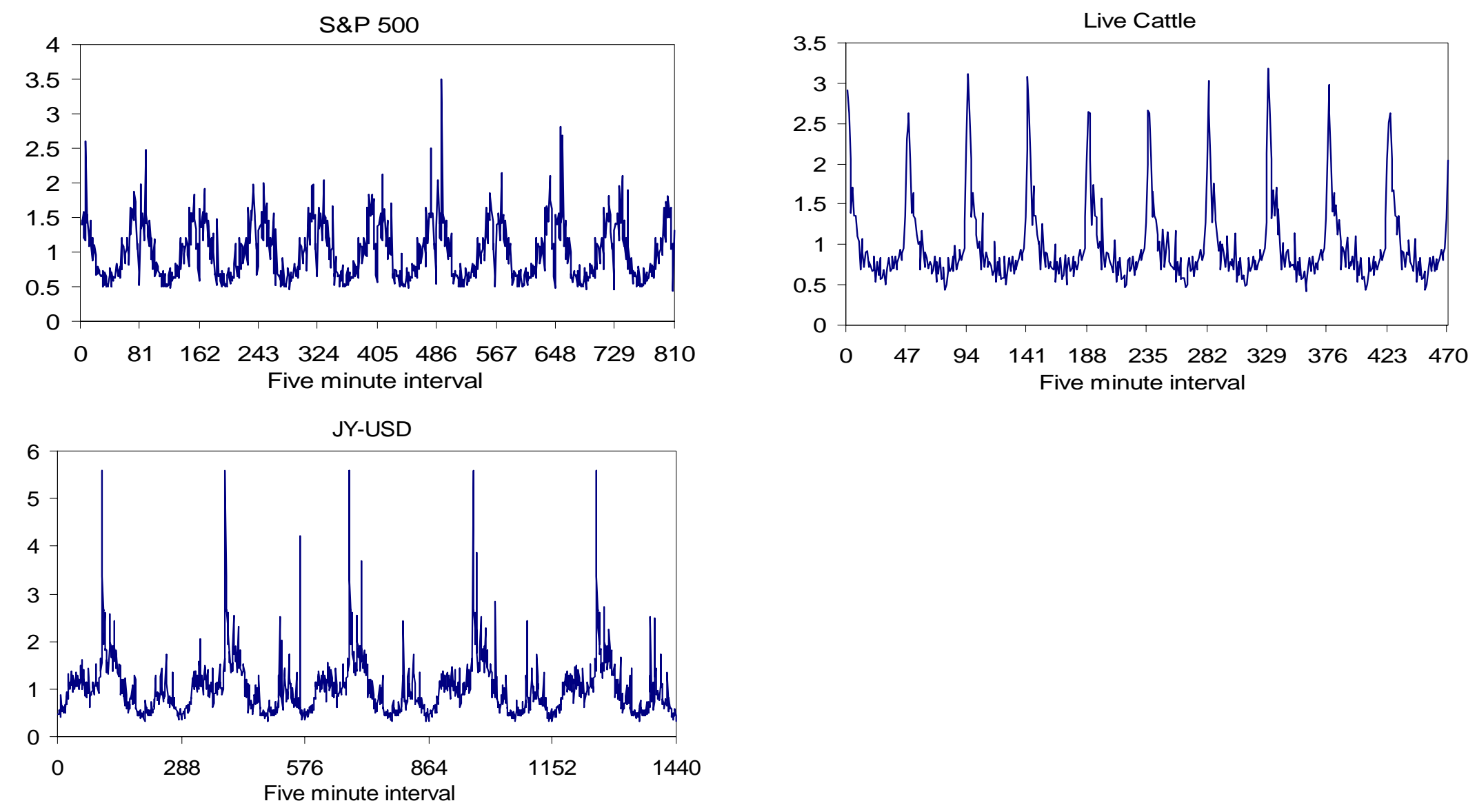


\section{Figure 1.5: Correlograms of the seasonal variances estimated by the ARCSV process}

Figure 1.5 includes the correlogram of the estimated seasonal volatility, relative to Figure 1.1 which graphs the correlogram of the unfiltered and filtered absolute returns. Hence, Figure 1.5 shows the behavior of the intraday seasonal variance component. The correlogram of the intraday seasonal variance component starts with positive values, turns to negative values in the middle of a cycle, and returns to positive values as the lag length approaches a complete one day seasonal lag. This behavior of the intraday seasonal variance component causes the regular fluctuation in the correlogram of the unfiltered absolute returns.
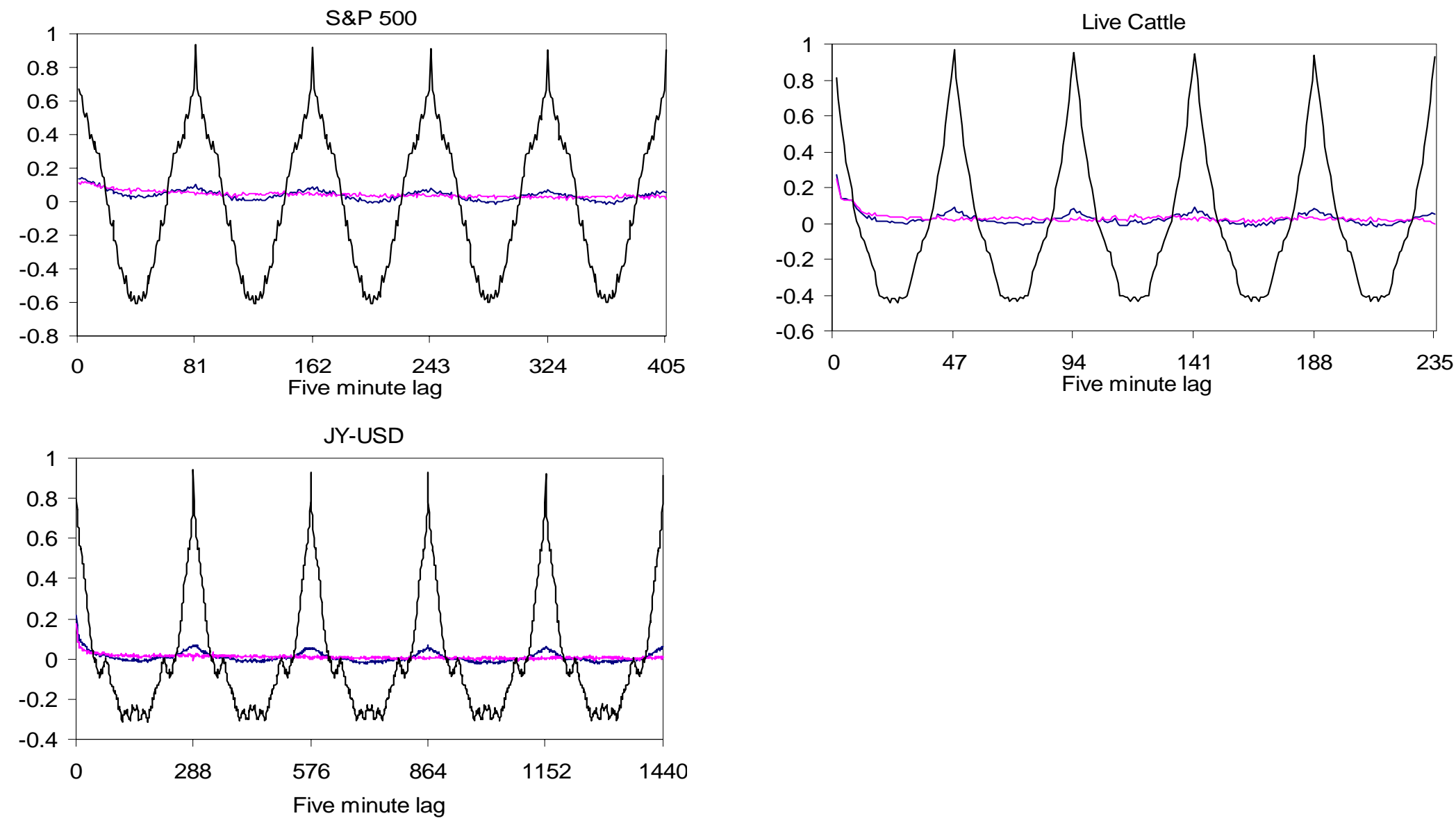
Figure 1.6: Spectral density of the estimated intraday seasonal variance

Figure 1.6 shows the spectral density of the estimated seasonal variance from zero to one. The estimated seasonal variance needs to show non-zero spectral density only at the seasonal periods. The seasonal periods are $81,81 / 2$, 81/3, etc., for the S7P 500 futures, 47, 47/2, 47/3, etc. for live cattle futures, and 288, 288/2, 288/3, etc for the JPY-USD exchange rate.
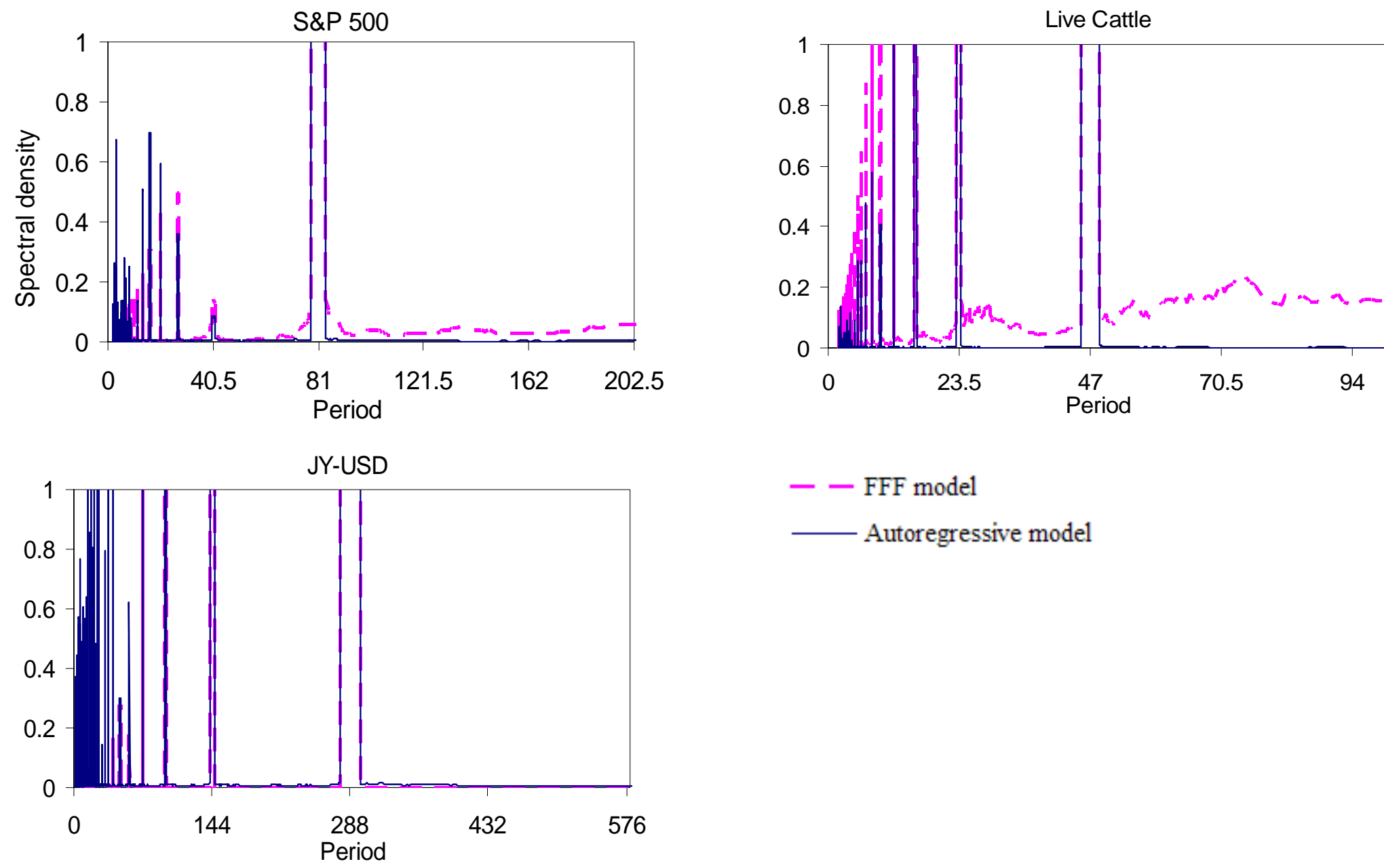

- - FFF model

- Autoregressive model 


\section{Figure 1.7: Spectral density of the unfiltered and filtered absolute returns}

Figure 1.7 graphs the spectral density of the unfiltered and filtered absolute returns. Spectral peaks exist for the spectral density of the unfiltered absolute returns at the seasonal frequencies but these peaks have to be removed in the associated filtered absolute returns. If the spectral densities of the filtered absolute returns continue to have peaks for the seasonal frequencies in the filtered absolute returns then this implies a maladjustment of the filtering process.
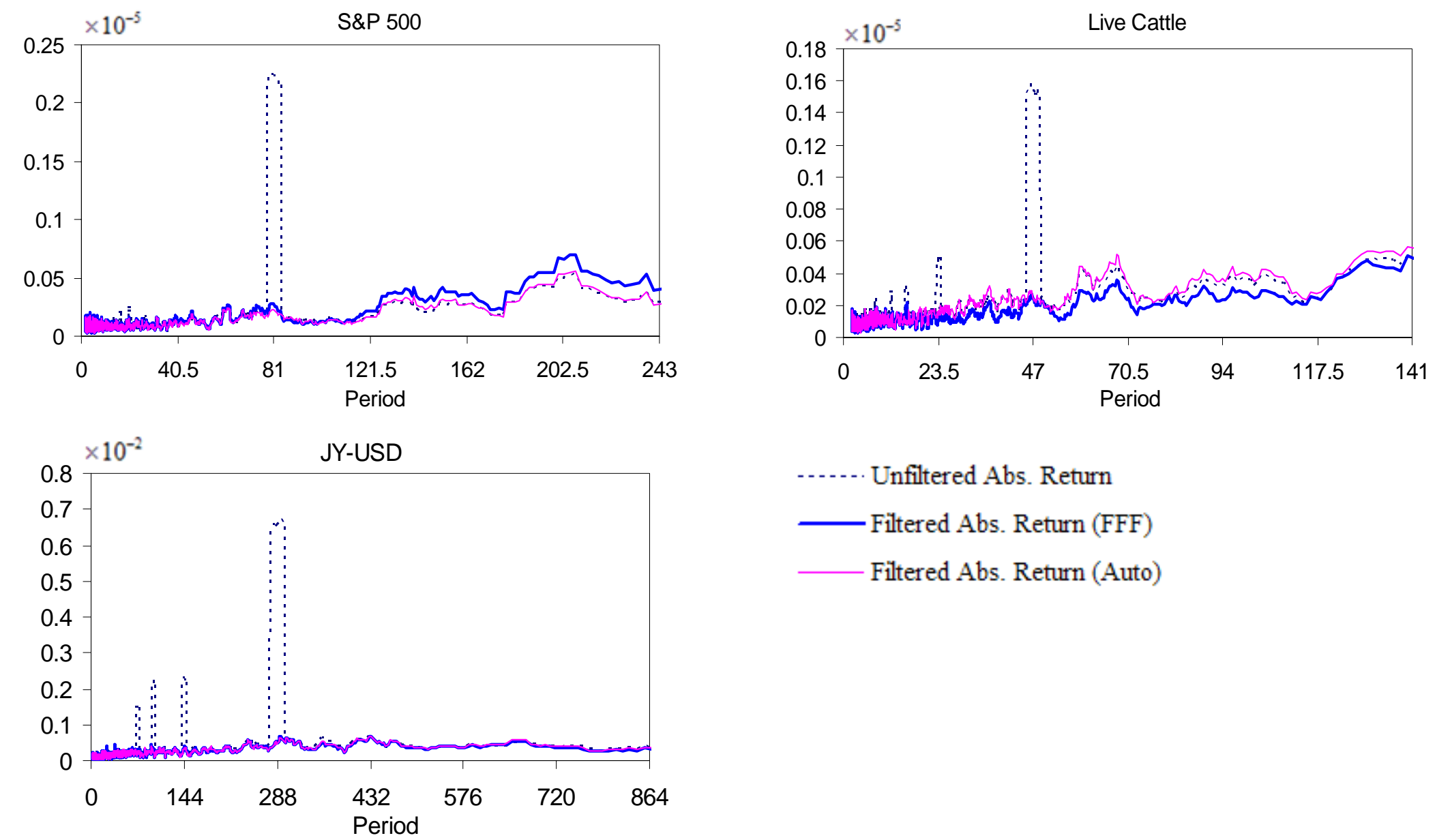

...... Unfiltered Abs. Return

- Filtered Abs. Return (FFF)

Filtered Abs. Return (Auto) 
Figure 1.8: Estimated coherence of the unfiltered and filtered absolute returns and their $99 \%$ confidence intervals - Coherence - - L LC and UC at $99 \%$ - Coherence 0.99
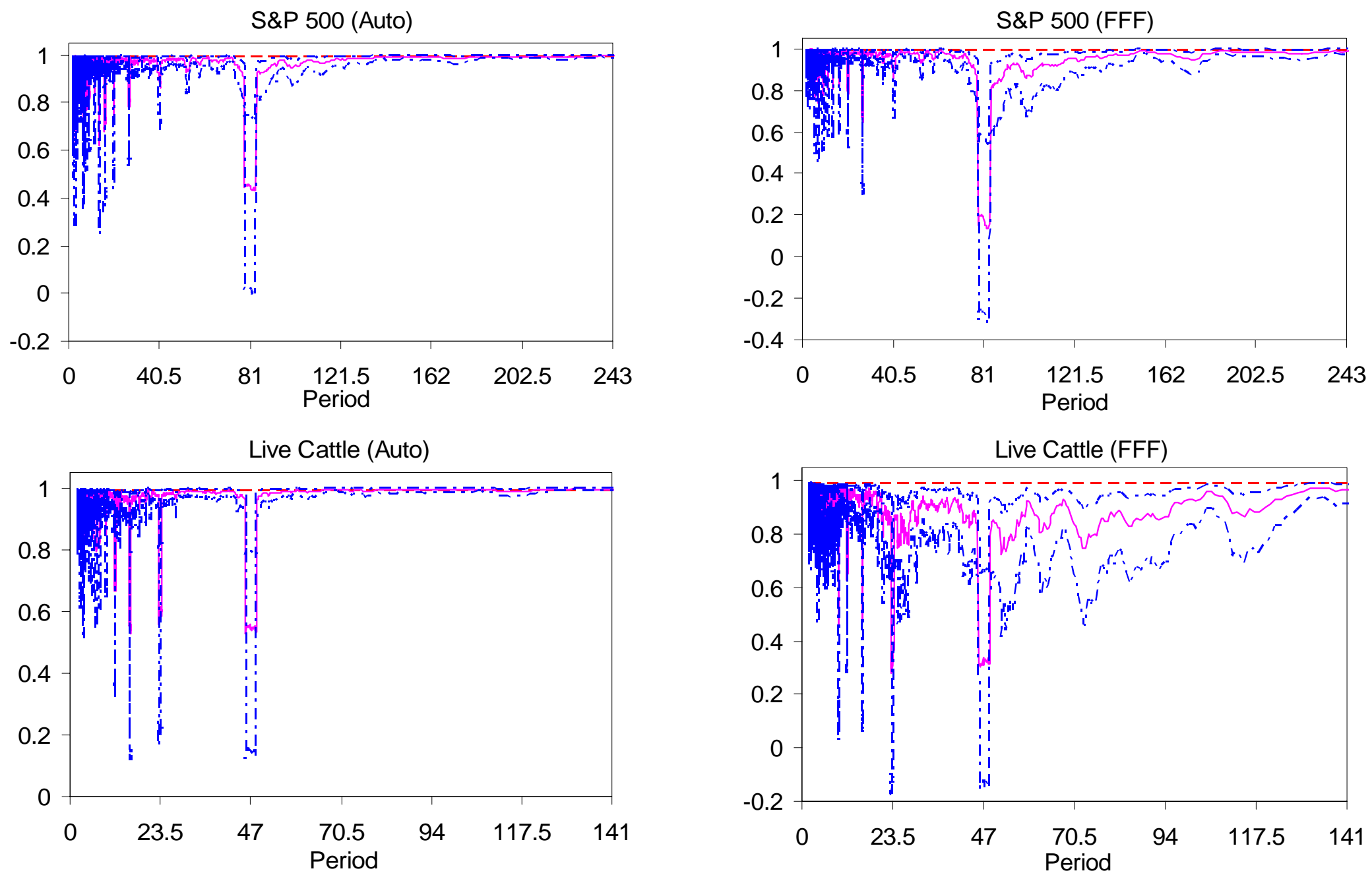
Figure 1.8: Estimated coherence of the unfiltered and filtered absolute returns and their $99 \%$ confidence intervals (Continued)

\section{Coherence - - LC and UC at $99 \%$ - Coherence 0.99}

Figure 1.8 graphs the estimated coherence of the unfiltered and filtered absolute returns and their $99 \%$ confidence intervals. Coherence is a measure of the correlation between two series as a function of the frequency. If the coherences between the unfiltered and filtered series are indistinguishable from 1.0 at the seasonal frequencies then a maladjustment in the deseasonality procedure exists. Because the variances at the non-seasonal frequencies should not be removed by the filtering process, the coherence of the unfiltered and filtered series should be 1.0 at the non-seasonal frequencies. If the coherence is statistically different from 1.0 at the non-seasonal frequencies then the filtering process has inappropriately removed variances of the series at the nonseasonal frequencies.
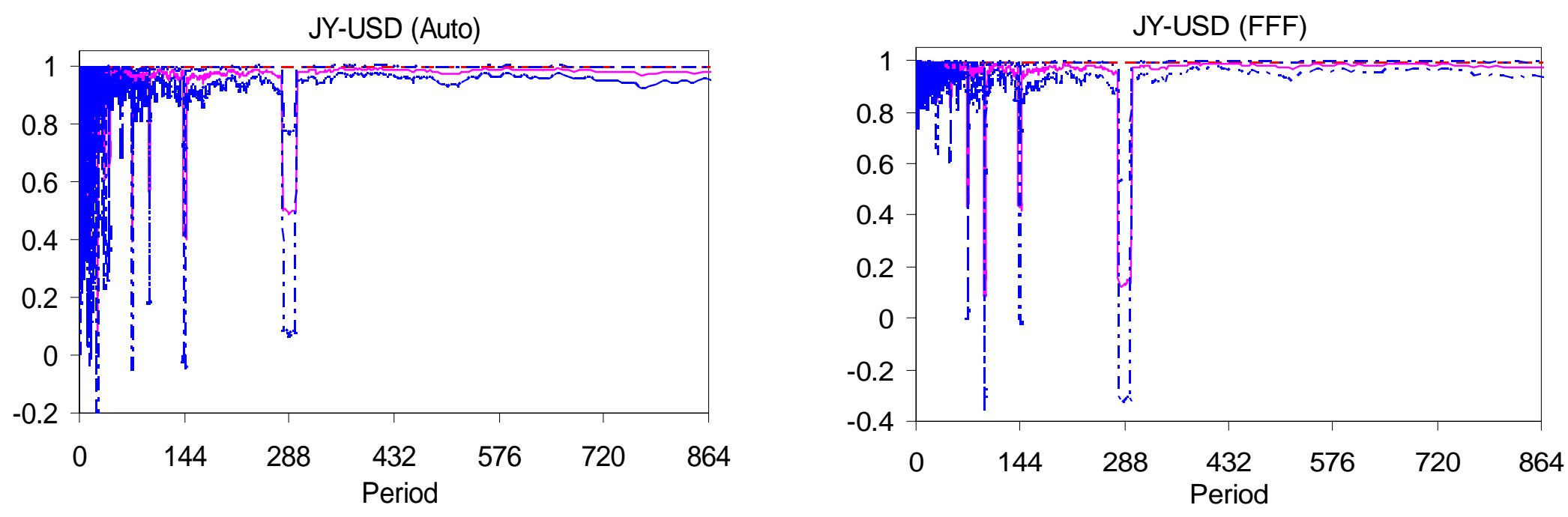
Figure 1.9: Estimated phase and $99 \%$ confidence intervals of the unfiltered and filtered absolute returns — Phase - - U UC and LC at $99 \%$
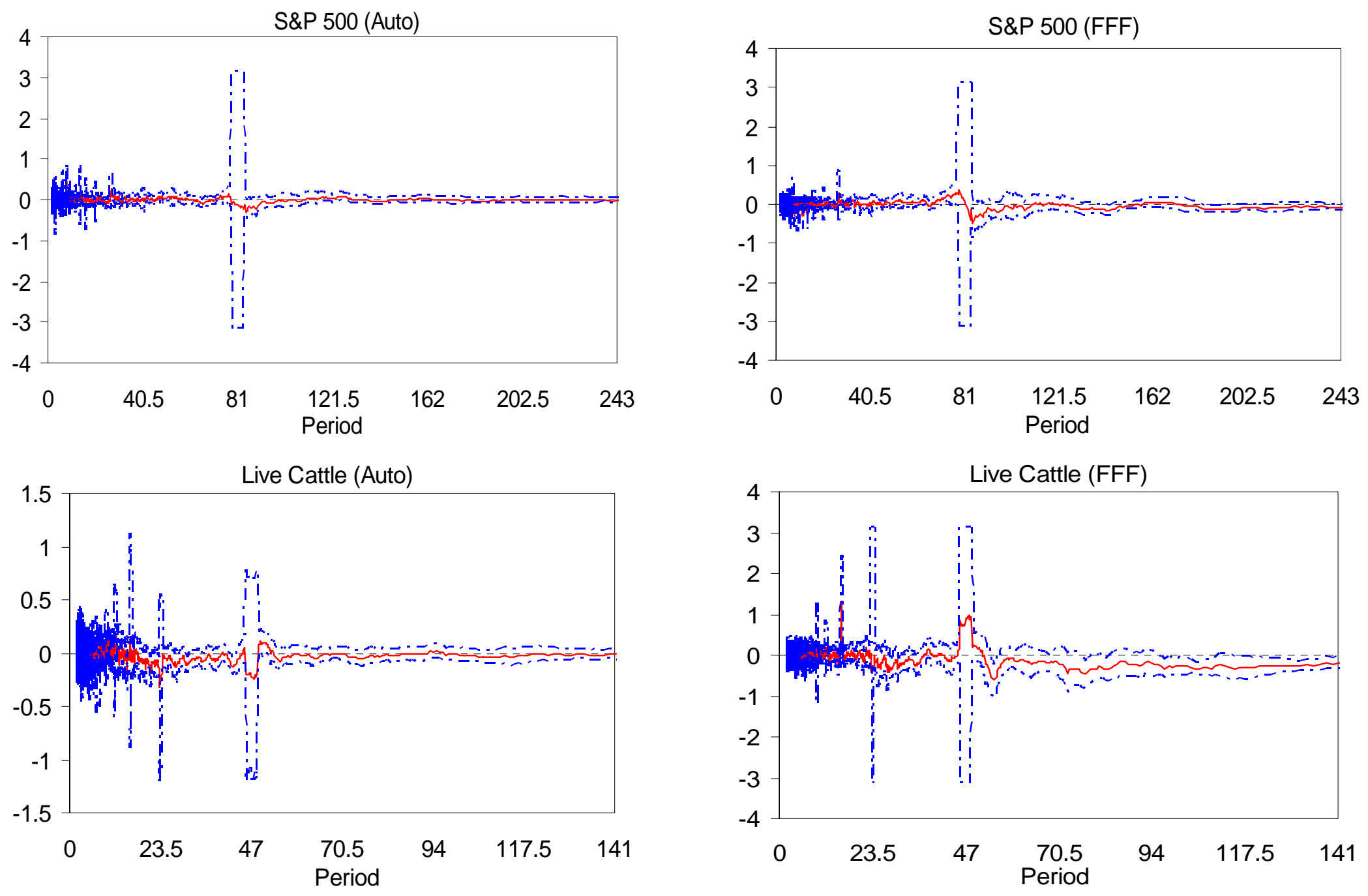
Figure 1.9: Estimated phase and $99 \%$ confidence intervals of the unfiltered and filtered absolute returns (Continued) Phase - - - UC and LC at $99 \%$

Figure 1.9 graphs the estimated phase for the S\&P 500 futures, live cattle futures, and JPY-USD spot exchange rate. The phase statistic estimates the phase shift between two series, i.e., the extent by which one series leads or lags another series. If there is a nonzero phase over the entire set of frequencies then a maladjustment has occurred.
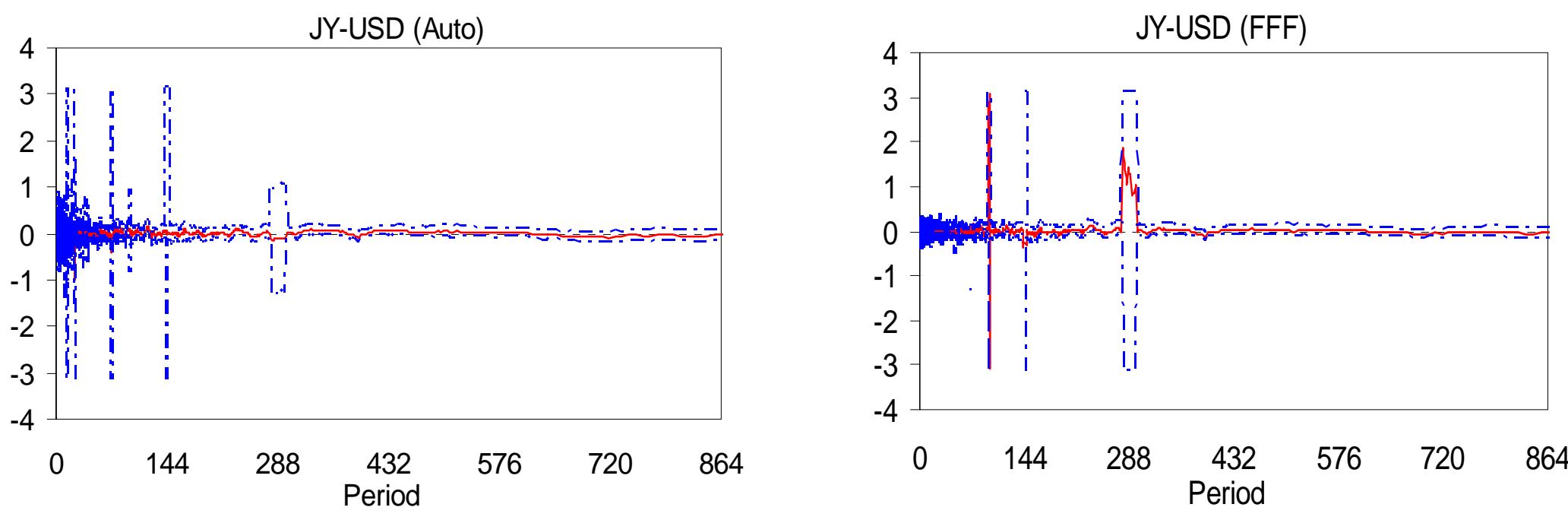
Table 1.3 : Estimation results of in-sample fit and encompassing regressions

The regression equations to examine the in-sample fit are $\log \left(r_{t, n}^{2}\right)=a_{0}+a_{1} \log \left(\hat{h}_{t} \hat{s}_{t, n}^{j}\right)+u_{t, n}, j=\{A R C S V$, Mean, FFF $\}$ and

$$
\begin{gathered}
\log \left(r_{t, n}^{2}\right)=b_{0}+b_{1} \log \left(\hat{h}_{t} \hat{s}_{t, n}^{\text {ARCV }}\right)+b_{2} \log \left(\hat{h}_{t} \hat{s}_{t, n}^{\text {Mean }}\right)+u_{t, n}, \log \left(r_{t, n}^{2}\right)=b_{0}+b_{1} \log \left(\hat{h}_{t} \hat{s}_{t, n}^{\text {ARCSV }}\right)+b_{2} \log \left(\hat{h}_{t} \hat{s}_{t, n}^{\text {FFF }}\right)+u_{t, n}, \\
\log \left(r_{t, n}^{2}\right)=b_{0}+b_{1} \log \left(\hat{h}_{t} \hat{s}_{t, n}^{\text {Mean }}\right)+b_{2} \log \left(\hat{h}_{t} \hat{s}_{t, n}^{\text {FFF }}\right)+u_{t, n} .
\end{gathered}
$$

where $\hat{s}_{t, n}^{A R C S V}, \hat{s}_{t, n}^{F F F}$ and $\hat{s}_{t, n}^{\text {Mean }}$ are the seasonal variance estimated by the ARCSV model, the FFF model without the interaction terms, and the mean of the normalized squared returns, respectively. If the ARCSV model estimates the seasonal variance better than the pure deterministic filtering models, then the parameter estimates of the ARCSV model will be larger in size and possess a greater statistical significance. In addition, the regression coefficient $\left(R^{2}\right)$ of the ARCSV model will be larger. In the following table, ARCSV, Mean, and FFF represents the coefficients of $\log \left(\hat{h}_{t} \hat{\hat{t}}_{t, n}^{\text {ARCSV }}\right)$, $\log \left(\hat{h}_{t} \hat{\hat{s}}_{t, n}^{\text {Mean }}\right)$, and $\log \left(\hat{h}_{t} \hat{s}_{t, n}^{F F F}\right)$.

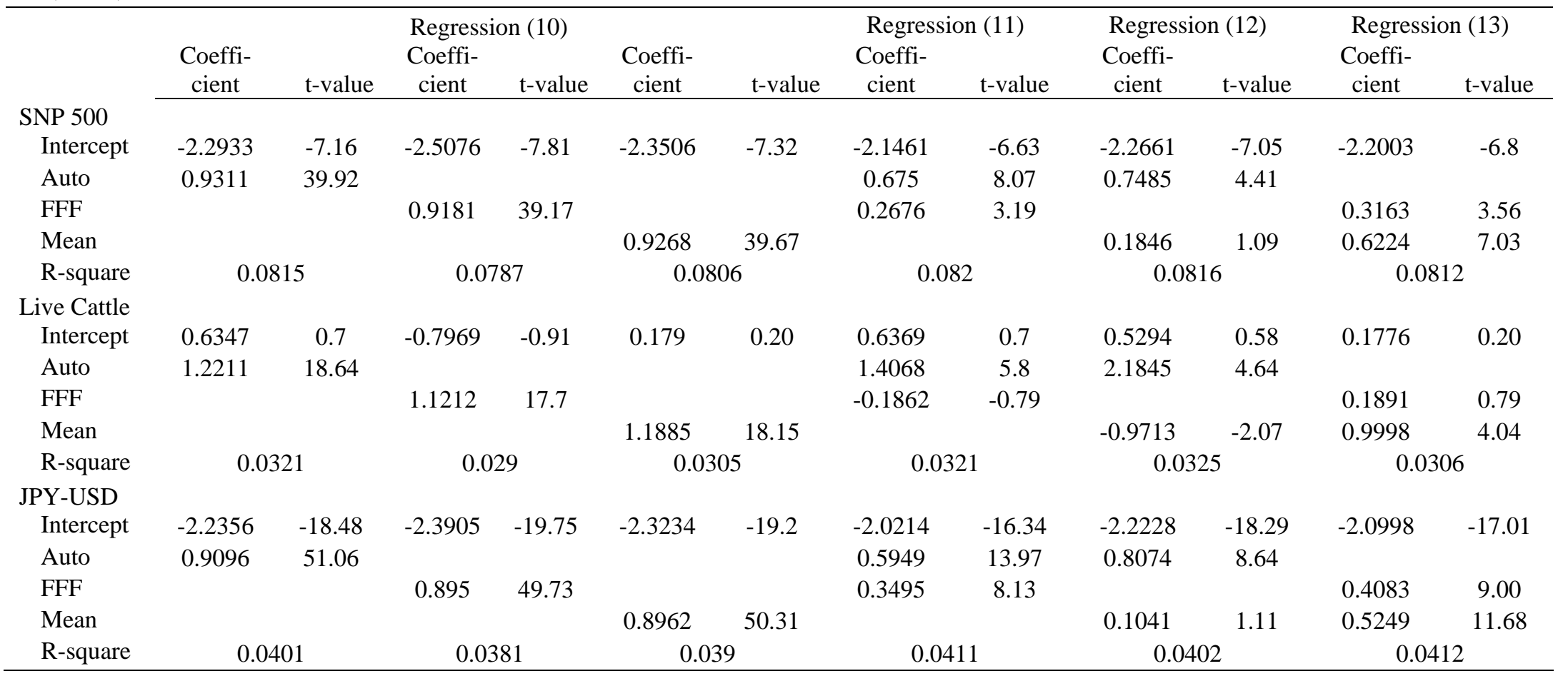




\section{REFERENCES}

Andersen, Torben G. and Tim Bollerslev, 1997a, Intraday periodicity and volatility persistence in financial markets, Journal of Empirical Finance 4, 115 - 158.

Andersen, Torben G. and Tim Bollerslev, 1997b, Heterogeneous information arrivals and return volatility dynamics: uncovering the long-run in high frequency returns, Journal of Finance 52, 975 - 1005.

Andersen, Torben G., Tim Bollerslev, Francis X. Diebold and Paul Labys, 2003, Modeling and forecasting realized volatility, Econometrica 71, 579 - 625.

Andersen, Torben G., Tim Bollerslev, Francis X. Diebold, and Heiko Ebens, 2001, The distribution of realized stock return volatility, Journal of Financial Economics 61, 43 -76 .

Beltratti, Andrea and Claudio Morana, 2001, Deterministic and stochastic methods for estimation of intra-day seasonal components with high frequency data, Economic Notes 30, $205-234$.

Bollerslev, Tim, 1986, Generalized autoregressive conditional heteroskedasticity, Journal of Econometrics 31, $307-327$.

Bollerslev, Tim and Eric Ghysels, 1996, Periodic autoregressive conditional heteroskedasticity, Journal of Business and Economic Statistics 14, 139 - 151.

Brockwell, Peter J. and Richard A. Davis, 1991, Time Series: Theory and Methods, $2^{\text {nd }}$ Ed.

Canina, Linda and Stephen Figlewski, 1993, The informational content of implied volatility, Review of Financial Studies 6, 659 - 681.

Cleveland, W. P. and G. C. Tiao, 1979, Modeling seasonal time series, Revue Economic Appliquee 32, 107 - 129.

Dacorogna, Michel M., Ulrich A. Müller, Robert J. Nagler, Richard B. Olsen, and Olivier V. Pictet, 1993, A geographical model for the daily and weekly seasonal volatility in the foreign exchange market, Journal of International Money and Finance 12, 413 438.

Daigler, Robert T., 1997, Intraday futures volatility and theories of market behavior, Journal of Futures Markets 17, 45 - 74. 
Daal, Elton, Joseph Farhat, and Peihwang P. Wei, 2006, Does futures exhibit maturity effect? New evidence from an extensive set of US and foreign futures contracts, Review of Financial Economics 15, 113 - 128.

Ding, Zhuanxin and Clive W. J. Granger, 1996, Modeling volatility persistence of speculative returns: a new approach, Journal of Econometrics 73, 185 - 215.

Engle, Robert E. 1982, Autoregressive conditional heteroskedasticity with estimates of the variance of United Kingdom inflation, Econometrica 50, 987 - 1007.

Engle, Robert F. and Kevin Sheppard, 2001, Theoretical and empirical properties of dynamic conditional correlation multivariate GARCH, Working paper, UCSD.

Engle, Robert F., 2002, Dynamic conditional correlation: a simple class of multivariate generalized autoregressive conditional heteroskedasticity models. Journal of Business and Economic Statistics 20, 339 - 350.

Engle, Robert, F., Magdalena E. Sokalska and Ananda Chanda, 2006, Forecasting intraday volatility in the US equity market - multiplicative component GARCH, Working paper, UCSD.

Engle, Robert, F. and Giampiero M. Gallo, 2003, A multiple indicators model for volatility using intra-daily data, Working paper, NYU.

Fuller, Wayne A., 1995, Introduction to Statistical Time Series, $2^{\text {nd }}$ Ed.

Gençay, Ramazan, Faruk Selçuk, and Brandon Whitcher, 2001, Differentiating intraday seasonalities through wavelet multi-scaling, Physica A 289, 543 - 556.

Gençay, Ramazan, Faruk Selçuk, and Brandon Whitcher, 2002, An Introduction to Wavelets and Other Filtering Methods in Finance and Economics.

Greene, William H., 2000, Econometric Analysis $4^{\text {th }}$ Ed.

Goodhart, Charles A. E. and Maureen O’Hara, 1997, High frequency data in financial markets: Issues and applications, Journal of Empirical Finance 4, 73 - 114.

Grossman, Sanford, 1976, On the efficiency of competitive stock markets where trades have diverse information, Journal of Finance 18, 81 - 101.

Harvey, Andrew, 1981, Time Series Models, Phillip Allan, Oxford.

Harvey, Andrew, Esther Ruiz, and Neil Shephard, 1994, Multivariate stochastic variance models, Review of Economics Studies 61, 247 - 264. 
Hughes, Michael, P. and Drew B. Winters, 2005, What is the source of different levels of time-series return volatility? The intraday U-shaped pattern or time-series persistence, Journal of Economics and Finance 29, $300-312$.

Hylleberg, Svend, 1986, Seasonality in Regression, Academic Press.

Jensen, Arne and Anders Cour-Harbo, 2001, Ripples in Mathematics - The Discrete Wavelet Transform.

Lockwood, Larry J., and Scott C. Linn, 1990, An examination of stock market return volatility during overnight and intraday periods 1964 - 1989, Journal of Finance 45, $591-601$.

Lutkepohle, Helmut, 2007, New introduction to multiple time series analysis, Springer.

Martens, Martin, Yuan-Chen Chang, Stephen J. Taylor, 2002, A comparison of seasonal adjustment methods when forecasting intraday volatility, Journal of Financial Research 25, 283 - 299.

Nerlove, Marc, 1964, Spectral analysis of seasonal adjustment procedures, Econometrica 32, $241-286$.

Omrane, Walid B. and Eric de Bodt, 2007, Using self-organizing maps to adjust for intraday seasonality, Journal of Banking and Finance 31, 1817 - 1838.

Tiao, George. C. and Michael R. Grupe, 1980, Hidden periodic autoregressive movingaverage models in time series data, Biometrika 67, 365 - 373.

Vecchia, Aldo V., 1985, Maximum likelihood estimation for periodic autoregressive moving average models, Techonometrics 27, 375 - 384.

Wood, Robert, Thomas H. McInish, and Keith Ord, 1985, An investigation of transactions data for NYSE, Journal of Finance 40, 723 - 739. 


\section{Appendix 1.1: Consistency and asymptotic normality of the parameters in the ARCSV process}

The residual $v_{t \mid n}$ in (3) contain the intraday heteroskedasticity variance component as assumed in Engle, Sokalska and Chanda (2006) and mentioned in Andersen and Bollerslev (1997a). Specifically, for a time period $\{t, n\}$ in (3),

$v_{t, n}=\sqrt{q_{t, n}} \xi_{t, n}$ and $\xi_{t, n} \sim$ iid $N(0,1)$

where $q_{t, n}$ represents the intraday heteroskedasticity variance component with the daily unconditional expectation being 1, i.e., $E\left[q_{t, n}\right]=1$ since the intraday conditional variance $q_{t, n}$ will not be relevant if returns are measured at a daily frequency. Then, the mean of $v_{t, n}$ given $I_{t-1, n}$ is

$$
E\left[v_{t, n} \mid I_{t-1, n}\right]=\sqrt{q_{t, n}} E\left[\xi_{t, n} \mid I_{t-1, n}\right]=0 \text {, }
$$

where $I_{t-1, n}$ be the information set at the day $\mathrm{t}-1$ for a particular $\mathrm{n}$. Suppose that the intraday heteroskedasticity process is as follows:

$$
q_{t, n}=\gamma_{0}+\gamma_{1} \frac{\varepsilon_{t, n-1}^{2}}{h_{t} S_{t, n-1}}+\gamma_{2} q_{t, n-1}
$$

By taking conditional expectation operator and substituting iteratively,

$$
E\left[q_{t, n} \mid q_{t, n-N}\right]=\frac{\gamma_{0}\left(1-\left(\gamma_{1}+\gamma_{2}\right)^{N}\right)}{1-\left(\gamma_{1}+\gamma_{2}\right)}+\left(\gamma_{1}+\gamma_{2}\right)^{N} q_{t, n-N}
$$

Or, because $q_{t, n-N}=q_{t-1, n}$,

$E\left[q_{t, n} \mid q_{t-1, n}\right]=\frac{\gamma_{0}\left(1-\left(\gamma_{1}+\gamma_{2}\right)^{N}\right)}{1-\left(\gamma_{1}+\gamma_{2}\right)}+\left(\gamma_{1}+\gamma_{2}\right)^{N} q_{t-1, n}$

where $E\left[\frac{\varepsilon_{t, n-1}^{2}}{h_{t} s_{t, n-1}} \mid q_{t, n-1}\right]=q_{t, n-1}$ is used. However, if $\mathrm{N}$ is large and $\gamma_{1}+\gamma_{2}<1$, then $q_{t, n}$ can be approximated by 
$E\left[q_{t, n} \mid q_{t-1, n}\right] \cong \frac{\gamma_{0}}{1-\left(\gamma_{1}+\gamma_{2}\right)}$

In this study, $\mathrm{N}$ is 81,47 and 288 respectively for S\&P500, live cattle futures contracts, and JPY-USD spot exchange rate. The above equation shows the expected value of the intraday heteroskedasticity. Hence,

$\lim _{N \rightarrow \infty} E\left[q_{t, n} \mid q_{t-1, n}\right]=\frac{\gamma_{0}}{1-\left(\gamma_{1}+\gamma_{2}\right)}=1$

or

$\operatorname{plim} q_{t, n}=1$.

If plim $q_{t, n}=1$ and $\xi_{t, n} \sim N(0,1)$, then the limiting distribution of $\sqrt{q_{t, n}} \xi_{t, n}$ is the distribution of $\xi_{t, n}$ (see Greene (2000), p. 115):

$v_{t, n} \equiv \sqrt{q_{t, n}} \xi_{t, n} \stackrel{d}{\longrightarrow} \xi_{t, n}$.

Hence, the asymptotic distribution of $v_{t, n}$ given $I_{t-1, n}$ is as follows.

$V_{t, n} \mid I_{t-1, n} \stackrel{a}{\sim}$ iid $N(0,1)$.

Given the above results, the estimators in (3) and (4) are consistent and follow the asymptotic normal distribution by the properties of the maximum likelihood estimators (see Greene (2000), p. 127). 


\section{Appendix 1.2: Covariance stationarity of the residual returns}

Seasonal variance process $\left(s_{t \mid n}\right)$ contains the deterministic part (unconditional seasonal variance component) which is captured by parameter $\alpha_{0 \mid n}$ and the stochastic part which is captured by $\alpha_{1 \mid n}$ and $\beta_{1 \mid n}$ together as shown in (4). The residual process $r_{t \mid n}$ is nonstationary because the unconditional variance component in the seasonal variance $\left(s_{t \mid n}\right)$ is time-varying. Note that if the unconditional variance of a series is not constant, then the series is nonstationary. The conditionally time-varying daily variance component $\left(h_{t}\right)$ is not a source of nonstationarity. (See Fuller, p. 111 and Enders, p. 155) It is because the process which is conditionally heteroskedastic can be unconditionally homoskedastic. For example, the GARCH process has a constant unconditional variance.

However, the residual returns $r_{t \mid n}$ is covariance stationary if the following conditions are met:

a. $E\left[r_{t \mid n}\right]<\infty$ and $E\left[r_{t \mid n}\right]=E\left[r_{t+h \mid n}\right]$ for all $h \in N$

b. $\operatorname{Cov}\left[r_{t \mid n}, r_{t+h \mid n}\right]<\infty$ and $\operatorname{Cov}\left[r_{t \mid n}, r_{t+h \mid n}\right]=\operatorname{Cov}\left[r_{t+g \mid n}, r_{t+h+g \mid n}\right]$ for all $\mathrm{h}$ and $\mathrm{g} \in N$

If the mean model in (14) is correctly specified, then $E\left[r_{t, n}\right]=0$ for all $\{t, n\}$, or $E\left[r_{t \mid n}\right]=0$ for all $n$. Hence, $E\left[r_{t \mid n}\right]=E\left[r_{t+h \mid n}\right]=0<\infty$ for all $h \in N$.

Hence, the first moment of $r_{t \mid n}$ is finite and constant.

Appendix 1 shows that $v_{t, n} \mid I_{t-1, n} \stackrel{a}{\sim}$ iid $N(0,1)$. Therefore,

$$
\begin{aligned}
& \operatorname{Cov}\left[\sqrt{h_{t} s_{t \mid n}} v_{t \mid n}, \sqrt{h_{t+h} s_{t+h \mid n}} v_{t+h \mid n}\right]=\sqrt{h_{t} s_{t \mid n}} \sqrt{h_{t+h} S_{t+h \mid n}} E\left[v_{t \mid n} v_{t+h \mid n}\right] \\
& =\sqrt{h_{t+g} s_{t+g \mid n}} \sqrt{h_{t+h+g} s_{t+h+g \mid n}} E\left[v_{t+g \mid n} v_{t+h+g \mid n}\right]=0<\infty
\end{aligned}
$$

for all $h \in N$ and $h \neq 0$.

$E\left[h_{t} S_{t \mid n} v_{t \mid n}^{2}\right]=\sigma \bar{s}_{t \mid n} E\left[v_{t \mid n}^{2}\right]=\sigma \bar{s}_{t+h \mid n} E\left[v_{t+h \mid n}^{2}\right]=\sigma \bar{s}_{t \mid n}<\infty$ for $h=0$, 
where $\sigma$ is the unconditional daily variance i.e., $E\left[h_{t}\right]=\sigma, \bar{s}_{t \mid n}$ is the unconditional seasonal variance at $\mathrm{n}$ i.e., $\bar{s}_{t \mid n}=\bar{s}_{t+h \mid n}=\alpha_{0, n} /\left(1-\alpha_{1, n}-\beta_{1 \mid n}\right)$. Hence, the covariance and variance of $r_{t \mid n}$ is finite and constant. 


\section{Appendix 1.3: Proof of Theorem 1}

As Nerlove (1964) note, the seasonality is defined as a characteristic of a time series that gives rise to spectral peaks at seasonal frequencies. Based on this definition of seasonality, I prove Theorem 1.

It is well known that the unconditional variance of a time series $\left(V_{\tau}\right)$ can be represented by the following spectral density form (See Fuller, 1995, p. 144):

$$
\begin{aligned}
& \sigma_{V}^{2}\left(\omega, \gamma_{i}\right)=\int_{\omega=-\pi}^{\pi} \frac{1}{2 \pi}\left(\gamma_{0}+2 \sum_{i=1}^{T N-1} \gamma_{i} \cos (\omega \cdot i)\right) d \omega, \\
& \gamma_{i}=\operatorname{cov}\left(V_{\tau}, V_{\tau+i}\right),
\end{aligned}
$$

where the $\tau=1, \cdots, T N$ is the cumulative intraday index with a day index $(t=0, \cdots, T-1)$ and an intraday index $(n=1, \cdots, N)$. That is, one day is composed of $N$ intraday periods. The above spectral representation in (A15) shows that the variance is decomposed by autocovariance $\left(\gamma_{i}\right)$ and frequency $(\omega)$. Let the time series be the variance series $\left(V_{\tau}\right)$ which has a daily periodic component (the intraday seasonal variance component). If a regular intraday seasonal component in a variance series is consistently present on a daily basis, such as the U-shape intraday volatility pattern, then the seasonal frequencies (periods) that contribute to the intraday seasonal component will be intraday frequencies $\frac{m}{T N}$ (intraday periods $\frac{T N}{m}$ ), where $m=\frac{N}{n}, n=1, \cdots, N \quad$. Let $\omega_{S}=\left\{\frac{2 \pi m}{T N} \mid m=\frac{N}{n}, n=1, \cdots, N\right\}$. The variances at the intraday frequencies are: 
$\sigma_{V}^{2}\left(\omega_{S}, \gamma_{i}\right)=\frac{1}{2 \pi}\left(\gamma_{0}+2 \sum_{i=1}^{P} \gamma_{i} \cos \left(\omega_{S} \cdot i\right)\right)$

The variances at the intraday frequencies in (A17) contain the daily periodic component and non-periodic component. Because the seasonal variance component is periodic on a daily basis, the autocovariances that contribute to the periodic component are $\gamma_{S}=\left\{\gamma_{i} \mid i=N, 2 N, \cdots, T N\right\}$. Therefore, the variances which reflect only daily periodic component at the intraday frequencies are represented by:

$$
\sigma_{V}^{2}\left(\omega_{S}, \gamma_{S}\right)=\frac{1}{2 \pi}\left(\gamma_{0}+2 \sum_{i \in I_{S}} \gamma_{i} \cos \left(\omega_{S} \cdot i\right)\right)
$$

where $I_{S}=\{N, 2 N, \cdots, T N\}$.

In order to show the $\operatorname{ARCSV}(p, q)$ in (3) and (4) has the spectral representation in (A18), I have to show the spectral densities of the $\operatorname{ARCSV}(p, q)$ is only the function of the intraday frequencies $\omega_{S}=\left\{\frac{2 \pi m}{T N} \mid m=\frac{N}{n}, n=1, \cdots, N\right\}$ and the periodic variance component $\gamma_{S}=\left\{\gamma_{i} \mid i=N, 2 N, \cdots, T N\right\}$.

Let $T_{\tau}=h_{t} S_{\tau}$ which is in (3), where $\tau=N t+n$. The spectral density of $T_{\tau}$ is obtained as follows:

$$
\begin{aligned}
f_{T}(\omega) & =\frac{1}{2 \pi} \sum_{\tau=1}^{N T} h_{t} s_{\tau} e^{-i \omega \tau} \\
& =\frac{1}{2 \pi} \sum_{\tau=1}^{N T}\left(\int_{\omega_{h}} f_{h}\left(\omega_{h}\right) e^{i \omega_{h} \tau} d \omega_{h}\right) s_{\tau} e^{-i \omega \tau} \\
& =\int_{\omega_{h}} f_{h}\left(\omega_{h}\right)\left(\frac{1}{2 \pi} \sum_{\tau=1}^{N T} s_{\tau} e^{-i\left(\omega-\omega_{h}\right) \tau}\right) d \omega_{h} \\
& =\int_{\omega_{h}} f_{h}\left(\omega_{h}\right) f_{S}\left(\omega-\omega_{h}\right) d \omega_{h},
\end{aligned}
$$


where $f_{h}\left(\omega_{h}\right)$ and $f_{s}\left(\omega-\omega_{h}\right)$ are the spectral densities of the daily variance $h_{t}$ and the seasonal variance $s_{\tau}$. Because $h_{t}$ is a daily variance, the frequency $\omega_{h}$ cannot be higher than one day. Hence the frequency $\omega-\omega_{h}$ represents the intraday frequency. For the discrete intraday time period $n=1, \cdots, N$, out of the total $T N$ cumulative time periods, the relevant periods are $T N, \cdots 2 N, N$. These periods are consistent with the frequencies $\frac{m}{T N}$ where $m=\frac{N}{n}, n=1, \cdots, N$.

We can express $\operatorname{ARCSV}(p, q)$ in the following vector form:

$S_{t}=D_{1,0}+\sum_{j=1}^{q} D_{1, j}{ }^{\circ} \tilde{\varepsilon}_{t-j} \tilde{\varepsilon}_{t-j}^{\prime}+\sum_{j=1}^{p} D_{2, j} S_{t-j}$

or

$\sum_{j=0}^{p} D_{2, j} S_{t}=D_{1,0}+\sum_{j=1}^{q} D_{1, j} \circ \tilde{\varepsilon}_{t-j} \tilde{\varepsilon}_{t-j}^{\prime}$

where $S_{t}=\operatorname{diag}\left\{s_{t, n}\right\}, D_{1, j}=\operatorname{diag}\left\{\alpha_{j \mid n}\right\}, D_{2, j}=\operatorname{diag}\left\{\beta_{j \mid n}\right\}, \tilde{\varepsilon}_{t-1}=\left(\tilde{\varepsilon}_{t-1||} \cdots \tilde{\varepsilon}_{t-1 \mid N}\right)^{\prime}$, 。 represents the Hadamard product which is computed by element by element multiplication. Fourier transform of (A20) is as follows (see Fuller, 1995, p. 179-180):

$$
f_{S}\left(\omega, \gamma_{i}\right)=f_{D_{2}}^{*-1}(\omega) f_{D_{1}}^{*}(\omega) \frac{1}{2 \pi}\left(\Gamma_{\tilde{\varepsilon}, 0}+2 \sum_{j=1}^{q} \Gamma_{\tilde{\varepsilon}, j} \cos (\omega \cdot j)\right) f_{D_{1}}(\omega) f_{D_{2}}^{-1}(\omega)
$$

or

$$
f_{S}\left(\omega, \gamma_{i}\right)=\frac{1}{2 \pi}\left(\Gamma_{S, 0}+2 \sum_{j=1}^{q} \Gamma_{S, j} \cos (\omega \cdot j)\right)
$$

where $f_{s}\left(\omega, \gamma_{i}\right)$ is the spectral density of $S_{t}$ as a function of frequency factor $\omega$ and autocovariance $\gamma_{i}, \Gamma_{\tilde{\varepsilon}, j} \equiv \operatorname{diag}\left\{E\left(\tilde{\varepsilon}_{t} \tilde{\varepsilon}_{t+j}^{\prime}\right)\right\}, \Gamma_{s, j} \equiv f_{D_{2}}^{*-1}(\omega) f_{D_{1}}^{*}(\omega) \Gamma_{\tilde{\varepsilon}, j} f_{D_{1}}(\omega) f_{D_{2}}^{-1}(\omega)$, 


$$
\begin{aligned}
& f_{D_{1}}(\omega)=\frac{1}{2 \pi} \sum_{j=1}^{q} D_{1, j} e^{-i \omega j}, \\
& f_{D_{1}}^{*}(\omega)=\frac{1}{2 \pi} \sum_{j=1}^{q} D_{1, j} e^{i \omega j}, \\
& f_{D_{2}}(\omega)=\frac{1}{2 \pi} \sum_{j=0}^{p} D_{2, j} e^{-i \omega j}, \text { and } \\
& f_{D_{2}}^{*}(\omega)=\frac{1}{2 \pi} \sum_{j=0}^{p} D_{2, j} e^{i \omega j} .
\end{aligned}
$$

The index $j=1, \cdots, p$ or $q$ in (A22) is day index $(t)$ and hence corresponds to $n=$ $N, 2 N, \cdots, p N$ or $q N$. These periods are the same as the index set $I_{S}=\{N, 2 N, \cdots, T N\}$. Thus, it follows that the spectral density of the $\operatorname{ARCSV}(p, q)$ includes only $\Gamma_{\tilde{\varepsilon}, j}$ where $j \in I_{S}$

As shown above, the $\operatorname{ARCSV}(p, q)$ is only the function of the intraday frequencies $\omega_{S}=\left\{\frac{2 \pi m}{T N} \mid m=\frac{N}{n}, n=1, \cdots, N\right\} \quad$ and the periodic variance component $\Gamma_{S}=\left\{\Gamma_{i} \mid i=N, 2 N, \cdots, T N\right\}$. 


\section{Appendix 1.4: The proof Theorem 2}

Intuitively, if the innovation and persistency terms in the ARCSV model are ignored then the remaining constant term will capture the unconditional mean of the seasonal variance for each intraday interval. Hence, the autoregressive model without the innovation and the persistency terms is indistinguishable from the currently used deterministic models. In the following sections I show that the FFF model and the variance mean filtering model are special cases of the autoregressive model, and that the variance mean filtering model better captures the seasonal variance than does the FFF model when a finite sample exists.

\section{A. The FFF model vs the autoregressive model}

Let $S_{t}=\left\{s_{t \mid 1}, \cdots, s_{t \mid N}\right\}$ be the pattern of the intraday seasonal variance series with the unconditional expectation, $E\left[S_{t}\right]=\left\{E\left[s_{t \mid 1}\right], \cdots, E\left[s_{t \mid N}\right]\right\}$. We can think of $E\left[s_{t \mid n}\right]$ as an observation at period of $\{\mathrm{t}, \mathrm{n}\}$ from a continuous function of $E\left[S_{\tau}\right]$. Let us assume that $E\left[S_{\tau}\right]$ is a continuous function and its $d E\left[S_{\tau}\right] / d \tau$ is square integrable, where the index $\tau$ is the cumulative intraday index defined as $\tau=N t+n$ for $t=0, \cdots, T-1$ and $n=1, \cdots, N$. Then there is a trigonometric polynomial $f_{\tau}$ of which $f_{\tau}$ at $n$ converges to the unconditional mean, $E\left[s_{t \mid n}\right]$, at $n$, absolutely and uniformly as follows (see Theorem 3.1.8 in Fuller (1995)):

$$
f_{\tau}=\bar{f}_{\tau}+\sum_{n=1}^{N / 2}\left(A_{n} \cos \frac{2 \pi n}{N} \tau+B_{n} \sin \frac{2 \pi n}{N} \tau\right) \text { if } N \text { is even, }
$$

or

$$
f_{\tau}=\bar{f}_{\tau}+\sum_{n=1}^{(N-1) / 2}\left(A_{n} \cos \frac{2 \pi n}{N} \tau+B_{n} \sin \frac{2 \pi n}{N} \tau\right) \text { if } N \text { is odd. }
$$

Now suppose that the conditional mean of $s_{t \mid n}$ follows an ARMA process, for simplicity, ARMA(1,1). Then the conditional expectation of $s_{t \mid n}$ is

$E_{t-1}\left[s_{t \mid n}\right]=E\left[s_{t \mid n}\right]\left(1-\alpha_{1 \mid n}-\alpha_{2 \mid n}\right)+\alpha_{1 \mid n} \tilde{\varepsilon}_{t-1 \mid n}^{2}+\beta_{1 \mid n} s_{t-1 \mid n}$.

For the sample,

$S_{t \mid n}=\mu_{n}\left(1-\alpha_{1 \mid n}-\alpha_{2 \mid n}\right)+\alpha_{1 \mid n} \tilde{\varepsilon}_{t-1 \mid n}^{2}+\beta_{1 \mid n} S_{t-1 \mid n}$, 
or $s_{t \mid n}=\alpha_{0 \mid n}+\alpha_{1 \mid n} \tilde{\varepsilon}_{t-1 \mid n}^{2}+\beta_{1 \mid n} s_{t-1 \mid n}$ as in (2),

where $\alpha_{0 \mid n}=\mu_{n}\left(1-\alpha_{1 \mid n}-\beta_{1 \mid n}\right)$ and $\mu_{n}=T^{-1} \sum_{t=0}^{T-1} \widetilde{\varepsilon}_{t \mid n}^{2}$ for $n=1, \cdots, N$.

Let $\mu_{n}(T) \equiv \mu_{n}=T^{-1} \sum_{t=0}^{T-1} \widetilde{\varepsilon}_{t \mid n}^{2}$. Assume that $\lim _{T \rightarrow \infty} \operatorname{Cov}\left[\mu_{n}(T), \tilde{\varepsilon}_{T \mid n}\right]=0$, then $\mu_{n}(T)$ converges to the unconditional mean $E\left[s_{t \mid n}\right]$ at $n$ as $T$ grows larger by the law of large number (see Theorem 6.1.1 in Fuller (1995)). Then, as $\mu_{n}(T)$ converges to $E\left[s_{t \mid n}\right], s_{t \mid n}$ converges to the conditional mean $E_{t-1}\left[s_{t \mid n}\right]$. Hence, it follows that the FFF model is a special case of the ARCSV process if the FFF model does not include the interaction terms, since the unconditional mean is obtained by limiting $\alpha_{1 \mid n}=\beta_{1 \mid n}=0$. Including the terms in the FFF model, such as, $n / N_{1}$ and $n^{2} / N_{2}$, does not change the above conclusion since those terms are only involved in capturing the unconditional mean for the deterministic patterns.

\section{B. The variance mean filtering model vs the autoregressive model}

The variance mean filtering model in Engle, et al. (2006) is also a special case of the autoregressive model when $\alpha_{1 \mid n}=\beta_{1 \mid n}=0$ in the autoregressive model. If $\alpha_{1 \mid n}=\beta_{1 \mid n}=0$ then the autoregressive model is $r_{t \mid n}=\sqrt{\hat{h}_{t} s_{t \mid n}} v_{t \mid n}$ where $s_{t, n}=\alpha_{0, n}$. By rearranging, the normalized return is obtained as $r_{t \mid n} / \sqrt{\hat{h}_{t}}=\sqrt{s_{t \mid n}} v_{t \mid n}=\sqrt{\alpha_{0 \mid n}} v_{t \mid n}$. The mean of the squared normalized returns for $n$ is $E\left[\left(r_{t \mid n} / \sqrt{\hat{h}_{t}}\right)^{2}\right]=E\left[\tilde{\varepsilon}_{t \mid n}^{2}\right]=E\left[s_{t \mid n} v_{t \mid n}^{2}\right]=\alpha_{0 \mid n} E\left[v_{t \mid n}^{2}\right]=\alpha_{0 \mid n}$. Hence, it follows that if $\alpha_{1 \mid n}=\beta_{1 \mid n}=0$ then $\hat{\alpha}_{0 \mid n}$ is the estimate $\left(T^{-1} \sum_{t=1}^{T} \tilde{\varepsilon}_{t \mid n}^{2}\right)$ of the unconditional mean $\left(E\left[s_{t \mid n}\right]\right)$ of the seasonal variance for each $n$ of the variance mean filtering model, where $E\left[\hat{\alpha}_{0 \mid n}\right]=\alpha_{0 \mid n}=E\left[s_{t \mid n}\right]$.

\section{The FFF model vs the variance mean filtering model}

In the limit, the seasonal variances estimated by the FFF model without the interaction term and by the variance mean filtering model at each $n$, are equal. It is shown 
above that if $\alpha_{1 \mid n}=\beta_{1 \mid n}=0$ then the constant $\hat{\alpha}_{0 \mid n}$ in the autoregressive model is the estimate of the unconditional mean $\left(E\left[s_{t \mid n}\right]\right.$ ) for the seasonal variance at $n$ of the FFF model and the variance mean filtering model. Therefore, as $T$ grows larger, those estimates of the seasonal variance from the FFF model without the interaction terms and the variance mean filtering model become equal. However, in the finite sample, while the variance mean filtering model allows independence between the means for different $n$, the FFF model does not allow independence. Therefore, in this case it is expected that the variance mean filtering model will provide better estimates for the unconditional seasonal variance $E\left[s_{t \mid n}\right]$ compared to the FFF model without the interaction terms.

\section{Noise injection of the FFF model in filtering process}

The flexible Fourier from (FFF) approach to filter the seasonal variance component includes the interaction terms between the daily volatility and the sinusoids (see Andersen and Bollerslev, 1997a). The following Lemma 1 shows that the interaction terms introduce the statistical noise in both the estimated seasonal variance series and the filtered non-seasonal variance series.

Lemma 1. the interaction terms between the daily volatility and the sinusoids in the FFF model injects the statistical noise in both the estimated seasonal variance series and the filtered non-seasonal variance series.

Proof

Proof of Lemma 1 is directly follows from the proof of Theorem 1 by showing that the seasonal variance estimated by the FFF model contains the non-periodic variance components and the variance components at the non-seasonal frequencies.

The FFF model is

$x_{\tau} \equiv 2 \log \left(\left|R_{t, n}-\bar{R}_{t, n}\right|\right)-\log \left(\sigma_{t}^{2}\right)+\log (N)$

$x_{\tau}=f_{\tau}\left(\theta \mid \sigma_{\tau}, n\right)+u_{\tau}$

$f_{\tau}=\sum_{j=0}^{J} \sigma_{t}^{j} z_{\tau}$

where $z_{\tau} \equiv\left[\mu_{0 j}+\mu_{1 j} \frac{n}{N_{1}}+\mu_{2 j} \frac{n^{2}}{N_{2}}+\sum_{i=1}^{D} \lambda_{i j} I_{n=d_{i}}+\sum_{i=1}^{p}\left(\gamma_{p j} \cos \frac{2 \pi p n}{N}+\delta_{p j} \sin \frac{2 \pi p n}{N}\right)\right]$.

The estimated seasonal variance is 
$S_{\tau}=\left(\frac{T \cdot N \exp \left(0.5 f_{\tau}\right)}{\sum_{\tau=1}^{T N} \exp \left(0.5 f_{\tau}\right)}\right)^{2}$

$z_{\tau}$ is obtained from the Fourier series approximation in (A30), and $J=1$ for simplicity. The interaction term is $T_{\tau} \equiv \sigma_{t} z_{\tau}$. As shown in the proof of Theorem 1 , the spectral density of $T_{\tau}$ is

$f_{T}(\omega)=\int_{\omega_{\sigma}} f_{\sigma}\left(\omega_{\sigma}\right) f_{z}(\omega) d \omega$

Given the fact that the interaction term is $T_{\tau} \equiv \sigma_{t} z_{\tau}$ is a component of the estimated seasonal variance $\left(s_{\tau}\right)$, the seasonal variance $\left(s_{\tau}\right)$ estimated by the FFF the variance components at the non-seasonal frequencies. Furthermore, the daily variance $\left(\sigma_{t}^{2}\right)$ contains the non-periodic variance components as shown in (A15) in Appendix 3. 


\section{Appendix 1.5: Proof of Theorem 3}

By the construction of the ARCSV filtering model in (4), the daily mean of the seasonal variance is 1 , as shown below:

From (3), the daily return is

$r_{t}=\sum_{n=1}^{N} r_{t \mid n}=N^{-0.5} \sqrt{h_{t}} \sum_{n=1}^{N} \sqrt{s_{t \mid n}} v_{t \mid n}$

The daily variance is2

$$
\begin{aligned}
& \begin{aligned}
E\left(r_{t}^{2} \mid I_{t-1}\right) & =h_{t} N^{-1} \sum_{n=1}^{N} s_{t \mid n} E\left(v_{t \mid n}^{2}\right) \\
& =h_{t} N^{-1} \sum_{n=1}^{N} s_{t \mid n} \\
& =h_{t}
\end{aligned} \\
& \text { or } \quad N^{-1} \sum_{n=1}^{N} s_{t \mid n}=1
\end{aligned}
$$

The probability limit of the equation in (A35) will be

$$
N^{-1} \sum_{n=1}^{N} s_{t \mid n} \stackrel{P}{\longrightarrow} E\left(s_{t \mid n}\right)=1
$$

The equation (A35) can be rewritten as

$$
\begin{aligned}
1 & =N^{-1} \sum_{n=1}^{N} s_{t \mid n} \\
& =N^{-1} \sum_{n=1}^{N}\left(\alpha_{0 \mid n}+\sum_{i=1}^{q} \alpha_{\| \mid n-i} \tilde{\varepsilon}_{t \mid n-i}^{2}+\sum_{j=1}^{p} \beta_{\| \mid n-j} s_{t \mid n-j}\right)
\end{aligned}
$$

As $\mathrm{N}$ grows larger, the probability limit of the equation in (A37) will be

$$
N^{-1} \sum_{n=1}^{N}\left(\alpha_{0 \mid n}+\sum_{i=1}^{q} \alpha_{1 \mid n-i} \widetilde{\varepsilon}_{t \mid n-i}^{2}+\sum_{j=1}^{p} \beta_{1 \mid n-j} S_{t \mid n-j}\right) \stackrel{P}{\longrightarrow} E\left(\alpha_{0 \mid n}\right)+\sum_{i=1}^{q} E\left(\alpha_{1 \mid n-i}\right) E\left(\widetilde{\varepsilon}_{t \mid n-i}^{2}\right)+\sum_{j=1}^{p} E\left(\beta_{1 \mid n-j}\right) E\left(s_{t \mid n-j}\right)
$$


Because $E\left(\widetilde{\varepsilon}_{t \mid n-i}^{2}\right)=E\left(s_{t \mid n-j}\right)=E\left(s_{t \mid n}\right)=1$, The above equation in (A38) can be rewritten as $N^{-1} \sum_{n=1}^{N}\left(\alpha_{0 \mid n}+\sum_{i=1}^{q} \alpha_{1 \mid n-i} \tilde{\varepsilon}_{t \mid n-i}^{2}+\sum_{j=1}^{p} \beta_{1 \mid n-j} s_{t \mid n-j}\right) \stackrel{P}{\longrightarrow} E\left(\alpha_{0 \mid n}\right)+\sum_{i=1}^{q} E\left(\alpha_{1 \mid n-i}\right)+\sum_{j=1}^{p} E\left(\beta_{1 \mid n-j}\right)=1$

The equation in (A39) shows that the $\operatorname{ARCSV}(q, p)$ filtering model captures the seasonal variance with weights of $\alpha_{0 \mid n}, \alpha_{1 \mid n-1}, \cdots, \alpha_{1 \mid n-q}, \beta_{1 \mid n-1}, \cdots, \beta_{1 \mid n-p}$ rather than only with $\alpha_{0 \mid n}$. Given the fact that $\alpha_{0 \mid n}$ is the estimate of the unconditional mean of the seasonal variance component and the sum of the weights of $\alpha_{0 \mid n}, \alpha_{1 \mid n-1}, \cdots, \alpha_{1 \mid n-q}, \beta_{1 \mid n-1}, \cdots, \beta_{1 \mid n-p}$ is unity, the increase in filtering performance of the $\operatorname{ARCSV}(q, p)$ is obtained as

$$
\sum_{i=1}^{q} E\left(\alpha_{1 \mid n-i}\right)+\sum_{j=1}^{p} E\left(\beta_{1 \mid n-j}\right)
$$

For a sample, the increase in filtering performance of the $\operatorname{ARCSV}(q, p)$ is

$$
N^{-1} \sum_{n=1}^{N}\left(\sum_{i=1}^{q} \alpha_{1 \mid n-i}+\sum_{j=1}^{p} \beta_{1 \mid n-j}\right)
$$




\section{Appendix 1.6: The test procedure for coherence}

Let $f_{11}(\omega)$ and $f_{22}(\omega)$ be smoothed spectral densities of unfiltered and filtered series, where $\omega$ represents frequency. Then the cross-spectral density $f_{12}(\omega)$ is expressed as $f_{12}(\omega)=C_{12}(\omega)-i q_{12}(\omega)=A_{12}(\omega) \exp \left(i \varphi_{12}(\omega)\right)$, where $A_{12}(\omega)=$ $\sqrt{C_{12}^{2}(\omega)+q_{12}^{2}(\omega)}=\left|f_{12}(\omega)\right|$ is the cross-amplitude spectrum, and $\varphi_{12}(\omega)=$ $\arctan \left(-i q_{12}(\omega) / C_{12}(\omega)\right)$ is the phase spectrum. Then, the coherence is obtained as

$$
\left|K_{12}(\omega)\right|=\frac{A_{12}(\omega)}{\sqrt{f_{11}(\omega) f_{22}(\omega)}}=\frac{\left|f_{12}(\omega)\right|}{\sqrt{f_{11}(\omega) f_{22}(\omega)}} .
$$

Drawing on Brockwell and Davis (1991, p. 150), the coherence $\left|K_{12}(\omega)\right|$ has the following confidence interval of an asymptotic normal distribution:

$$
\left(\tanh \left(\operatorname{arctanh}\left(\left|\hat{K}_{12}(\omega)\right|\right)-Z_{1-\alpha / 2} a / \sqrt{2}\right), \tanh \left(\operatorname{arctanh}\left(\left|\hat{K}_{12}(\omega)\right|\right)+Z_{1-\alpha / 2} a / \sqrt{2}\right)\right)
$$

where $a^{2}=\sum_{j=-d}^{d} W^{2}(j)$, the $W(j)$ is the weighting function with the bandwidth $2 d+1$ such that $\sum_{j=-d}^{d} W(j)=1$. For a 95\% confidence interval ( $\left.\alpha=0.05\right), Z_{1-\alpha / 2}=1.96$. However, the above confidence interval is inoperable for the null hypothesis that the coherence $\left|K_{12}(\omega)\right|$ is equal to 1 . This is because for an input $x=1$, the $\operatorname{arc} \tanh (1)=\infty$. Both the upper and lower confidence bounds converge to infinity. Hence, instead of testing the null hypothesis that $\left|K_{12}(\omega)\right|$ is equal to 1 , I test the following null hypothesis to make inferences on the statistical significance of the coherence. The null hypothesis is

$$
H_{0}:\left|K_{12}(\omega)\right|=0.99
$$

If the upper confidence bound lies below 0.99 then the null hypothesis $H_{0}$ is rejected, since this means that the unknown coherence is smaller than 0.99 . 


\section{Essay 2}

\section{Informational Decompositions of the Variance of Returns}

\subsection{Introduction}

This study investigates the time-varying informativeness of the price series, which can be employed to examine the deviation of actual price from the true price, such as when bubbles and crashes are being investigated. This study employs data from the major U.S. stock indexes during the late 1990s and early 2000s to examine such a case of a bubble and subsequent crash. In the noisy rational expectations models of Diamond and Verrecchia (1981), Admati (1985), Kim and Verrecchia (1991), and Shalen (1993), traders' reactions to price changes are decomposed into the components of unexpected information content and the effect of uninformed noisy traders' reactions to market activity. Uninformed traders react both to the price changes caused by information arrivals and to the price changes caused by other aspects of noise (liquidity demand), since they are uninformed and cannot distinguish between these factors. Hence, the changes in price are caused by both information arrivals and noise.

This study develops a new methodology to decompose the variance of returns into information and noise, given that both variances are conditional on time. One important condition for the study of the market microstructure, is that both the informational and noise variances must be conditional on time in order to reflect heteroskedastic informational arrivals and the dynamic behavior of noise throughout time. Previous studies do not allow heteroskedasticity over time. 
Variance decomposition into information and noise with time varying variances provides the following new findings. The first order moving average model employed in this study captures the influence of noise factors which is reflected in the time-varying moving average coefficient of the model. If the noise factor influences prices to increase, then the moving average coefficient becomes statistically positive, and vise-versa. In fact, the empirical results show that if the moving average coefficient is statistically positive for an extended period of time then prices are generating a bubble. ${ }^{31}$ If the moving average coefficient becomes statistically negative, which happens in 2002, 2003 and 2004, then the stock indexes reach a minimum value when the moving average coefficients are negative. The methodology developed here to decompose the variance into information and noise provides many possible research avenues to examine price behavior, such as investigating price bubbles, forecasting long-run price movements, and analyzing market microstructure issues such as the volume-volatility relation. ${ }^{32}$

Section 2.2 discusses previously developed models and their empirical limitations in capturing conditional information arrivals. Section 2.3 introduces an econometric model for variance decomposition by information and noise. In addition, the time varying measure of price informativeness and its test are developed in this section. Section 2.4

31 [This is not a footnote.] From p. 84, "if $\sigma_{z f_{\tau-1}}<0<\sigma_{z}^{2}$ and $\left|\sigma_{z f_{\tau-1}}\right|>\sigma_{z}^{2}$, then $\theta_{\tau-1}=-\left(\sigma_{z}^{2}+\sigma_{z f_{\tau-1}}\right) / \sigma_{u_{\tau-1}}^{2}>0$ in (10). In other words, if the uninformed speculators cause a price to move in the same direction as the past price change $\left(u_{\tau-1}\right)$, then this uninformed speculators' effect is opposite the information content, as shown by $\sigma_{z f_{\tau-1}}<0$. Therefore, if the negative $\sigma_{z f_{\tau-1}}$ value continuously becomes more negative for a prolonged time period $\left(\sigma_{z f_{s}}<\sigma_{z f_{\tau}}<0\right.$ for all $s>\tau$ ), then this should reflect a price that has deviated from its fair price.”

32 [Better delete this FN.] The additional volatility caused by the excess covariance between the expected returns (or information component) and the noisy demand corresponds to the measure of noise variance in this study. 
describes the data for this study. Results from the model introduced in section 2.3 are discussed in section 2.5. The conclusions follow in section 2.6.

\subsection{Concepts and literature review}

This study examines information arrivals and noise. Informationally efficient markets reflect new information swiftly so that price shows a sudden change for each new arrival of information. If the market is not informationally efficient, then new information is reflected over multiple time periods. Such a multiple time period adjustment leads to prices being positively autocorrelated. On the other hand, if information creates an overreaction that is reflected in prices then price changes are negatively autocorrelated. In addition to the slow- or over-reaction of information into prices, noise factors can cause prices to be either positively or negatively autocorrelated.

${ }^{33}$ Without any a priori knowledge about whether price changes are due to information or to noise, it is not possible to distinguish between (1) autocorrelation caused by information which is only partially reflected in the price, and (2) autocorrelation caused by non-informational noise factors such as autocorrelated liquidity-motivated trading, bid-ask spread bounce, uninformed feedback trading, etc. When these autocorrelationrelated factors, as listed in (1) and (2), have a minimal effect on the current price then price changes should more closely follow a random walk process. A shortcoming of such an analysis is that information not fully reflected in prices, and which causes price

\footnotetext{
${ }^{33}$ Autoregressive processes can be represented by a moving average process.
} 
changes to be autocorrelated, is treated as noise. ${ }^{34}$ Hasbrouck (1991a, 1991b) develops models which decompose the informational variance component of price change into a component caused by trading volume and a component caused by non-trading factors. ${ }^{35}$ The variance of the information (random walk) component of price change is computed from the moving average process of homoskedastic independent innovations of the returns (see equations (A4) and (A12) of Appendix 1). However, this formulation (the Wold decomposition of a stationary process) can not accommodate the heteroskedasticity of price changes (returns), which is a problem because conditional heteroskedasticity is pervasive in financial time series. ${ }^{36}$ In order to deal with this issue I model a moving average process where residuals are heteroskedastic (see (6) and (14) in Section 2.3.1). Therefore, I assume that the coefficient of the moving average is time-varying, so that price changes can be associated with a changing portion of the random walk (information) component (see (14) in Section 3.3).

Harvey (1989) and Fuller (1995) introduce a structural model which decomposes the variance of price changes into a random walk (information) component and a noise component. The noise component is assumed to be uncorrelated, while Hasbrouck allows the noise to be correlated. The model for the variance decomposition by information content in Section 2.3.1 is based on the structural model in Harvey and Fuller. I make two

\footnotetext{
${ }^{34}$ Hasbrouck (1991b, p. 573) discusses the price series associated with information that is immediately reflected in price as forming a random walk, which is referred to as the efficient or true price.

${ }^{35}$ If price and trading activity are examined with Vector AutoRegression, then the portion due to trading in the variance of the information (random walk) component of price is referred to as the informativeness of the trade (Hasbrouck 1991b). Hasbrouck does not have a noise component. The procedure in Hasbrouck (1991a and 1991b) to derive the informational variance component from a univariate and multivariate time series is explained in Appendix 1.

${ }^{36}$ Bera, Higgins, and Lee (1992) show that conditional heteroskedasticity is present if the coefficients of the autoregressive terms of the dependent variable and residual errors are random.
} 
alterations to the Harvey (1989) and Fuller (1995) models such that (1) the moving average is heteroskedastic, and (2) the coefficient of the moving average is time-varying. These two alterations enable one to produce the changing variance series of both the informational component and the noise component. In order to examine the relative size of the variance components, a time-varying measure of price informativeness is also developed.

\subsection{Models for variance decomposition}

\subsubsection{Heteroskedastic variance decomposition by information content and development of a time-varying measure of price informativeness}

This section explains how the structural model decomposes the variance into the informational component and the noise component, with the improvements previously discussed. The structural model is based on the following two equations: the observed price equation described by (1) and the unobserved random walk price equation given by

$$
p_{\tau}=m_{\tau}+z_{\tau}
$$

$m_{\tau}=m_{\tau-1}+f_{\tau}$

where $p_{\tau}=\log \left(P_{\tau}\right)$ and $P_{t}$ is the observed price. ${ }^{37}$ The $m_{\tau}$ variable in (1) is the efficient price, which follows a random walk, as described in (2). ${ }^{38}$ The $z_{\tau}$ variable is the

\footnotetext{
${ }^{37}$ As Lo and MacKinlay (1988) point out, there is a distributional advantage to using the log price in making statistical inferences, since the log price follows a normal distribution. Also, the difference of the $\log$ prices describes the return for the price series. Hasbrouck (1991b) also employs the log price in his model.
} 
component of price that represents the uncorrelated noise over time, and is orthogonal to the efficient price $\left(m_{\tau}\right)$, such that $z_{\tau} \sim N\left(0, \sigma_{z}^{2}\right)$. The noisy price change $\left(z_{\tau}\right)$ is caused by transient microstructure imperfections, liquidity-motivated traders. ${ }^{39}$ The uninformed profit-motivated speculator's demand for a risky asset is a function of noise as well as a function of the speculator's expected price change. Since uninformed speculators are not able to distinguish noise from the information component $\left(f_{\tau}\right)$, the speculators cause the non-zero covariance between the information component and the noise component. ${ }^{40}$ The $f_{\tau}$ is the uncorrelated residual of the efficient price, such that $f_{\tau} \sim N\left(0, \sigma_{f \tau}^{2}\right)$, and $\operatorname{Cov}\left(z_{\tau}, f_{s}\right)=\sigma_{z f_{\tau}}$ for $\tau=s$ and $\operatorname{Cov}\left(z_{\tau}, f_{s}\right)=0$ for $\tau \neq s .{ }^{41}$ Hence, $\sigma_{f_{\tau}}^{2}$ is the variance of the efficient price, while $\sigma_{z}^{2}$ represents the variance of noise. $\sigma_{z f_{\tau}}$ is the excess covariance between information and noise. In this model the heteroskedasticity of the variance of price is caused by two factors: information arrivals $\left(\sigma_{f_{\tau}}^{2}\right)$ and the covariance between noise trading and price change $\left(\sigma_{z f_{\tau}}\right)$. Moreover, the sum of $\sigma_{z}^{2}$ and $\sigma_{z f_{\tau}}$ is the measure of noise variance, since $\sigma_{z f_{\tau}}$ arises from the existence of liquidity-motivated

\footnotetext{
${ }^{38}$ The price series that follows a random walk is variously named as the "true price", the "efficient price", the "value (or fair) price" or the "consensus price" (see Hasbrouck (2002), p. 330)

${ }^{39}$ Hasbrouck (1993) notes that transient microstructure imperfections are caused by inventory control, price discreteness, etc.

${ }^{40}$ Note that the liquidity-motivated traders introduce a noise component which is denoted by $Z_{\tau}$, whereas uninformed speculators cause the covariance between the information component and the noise component which is denoted by $\sigma_{z f_{\tau}}$. See Shalen (1993) concerning the excess covariance between the information component and the noise component. In Shalen's (1993) model, noise is introduced by hedgers' random demand, whereas the excess covariance between the information component and the noise component is caused by uninformed speculators.

${ }^{41}$ See Harvey (1989, p. 112) for the variance structure of information and noise.
} 
traders and uninformed speculators. ${ }^{42}$ Therefore, both the measure of informational variance $\left(\sigma_{f_{\tau}}^{2}\right)$ and the measure noise variance $\left(2 \sigma_{z}^{2}+2 \sigma_{z f_{\tau}}\right)$ are time-varying.

The following explains that if information and noise satisfy the properties described in (1) and (2), as well as their distributional assumptions, then the difference in the observed price $\left(p_{\tau}-p_{\tau-1}\right)$, i.e. the return series $\left(r_{\tau}\right)$, can be represented by a moving average of order one. Based on my first order moving average model, along with the relation between the unobservable component and moving average component described by (8) and (9), the variance of the returns is decomposed into the informational variance component (from the price innovations) and the noise variance component (reflected in the moving average coefficient). In addition, the time-varying informativeness measure of the univariate time series is derived, where the measure of informativeness is defined as the variance of the efficient price divided by the total variance. The first difference of $p_{t}$ in (1) has the following form:

$$
\begin{aligned}
r_{\tau} & =m_{\tau}-m_{\tau-1}+z_{\tau}-z_{\tau-1} \\
& =f_{\tau}+z_{\tau}-z_{\tau-1}
\end{aligned}
$$

where $r_{\tau}=p_{\tau}-p_{\tau-1}=\log \left(P_{\tau}\right)-\log \left(P_{\tau-1}\right)$, and which has the following properties:

$$
\begin{aligned}
\operatorname{Var}\left(r_{\tau}\right)=\sigma_{f_{\tau}}^{2}+2 \sigma_{z_{\tau}}^{2}+2 \sigma_{z f_{\tau}} \\
\begin{aligned}
\operatorname{Cov}\left(r_{\tau}, r_{\tau-1}\right) & =E\left(\left(f_{\tau}+z_{\tau}-z_{\tau-1}\right)\left(f_{\tau-1}+z_{\tau-1}-z_{\tau-2}\right)\right) \\
& =E\left(-z_{\tau-1}^{2}-z_{\tau-1} f_{\tau-1}\right) \\
& =-\sigma_{z}^{2}-\sigma_{z f_{\tau-1}},
\end{aligned}
\end{aligned}
$$

${ }^{42}$ Beveridge and Nelson (1981) restrict their model to a trend and irregular components that are uncorrelated. However, this study allows the covariance to be any value. 
$\operatorname{Cov}\left(r_{\tau}, r_{\tau-1}\right)=0$ for $|h|>1$

The above shows that $r_{\tau}$ is described by a moving average of order one. Hence, $r_{\tau}$ can be rewritten as follows:

$r_{\tau}=\theta_{\tau-1} u_{\tau-1}+u_{\tau}$

$\theta_{\tau-1}=\frac{\operatorname{Cov}_{\tau-1}\left[r_{\tau}, u_{\tau-1}\right]}{\operatorname{Var}_{\tau-1}\left[u_{\tau-1}\right]}$

where $u_{\tau}$ is uncorrelated but is allowed to be heteroskedastic. It is well-known that the residuals $\left(u_{\tau}\right)$ of a moving average process can be heteroskedastic and the resultant parameter estimators are consistent. ${ }^{43}$ The moving average coefficient $\left(\theta_{\tau}\right)$ is assumed to be stochastic to accommodate the time-varying informativeness in price changes $\left(r_{\tau}\right)$, where $\operatorname{Cov}_{\tau-1}[\cdot]$ and $\operatorname{Var}_{\tau-1}[\cdot]$ are the conditional covariance and the conditional variance operators at period $\tau-1$, obtained by an ARMA process. If the covariance and variance in (7) converges to their unconditional means in probability, then the moving average coefficient $\left(\theta_{\tau}\right)$ will also converge to its unconditional mean in probability, which means that $\theta_{\tau}$ is a consistent estimator, while the sign of $\theta_{\tau}$ is an empirical issue. If the covariance between the random walk component $\left(f_{\tau}\right)$ and the noise component is sufficiently negative, then $\theta_{\tau}$ can be positive; see (9) below.

Since $r_{\tau}$ and $u_{1}\left(=r_{1}\right)$ are given values then $\theta_{\tau}$ and $\sigma_{u \tau}^{2}$ can be determined. The first order moving average $r_{\tau}$ has the following properties:

$\operatorname{Var}\left(r_{\tau}\right)=\sigma_{u_{\tau}}^{2}+\theta_{\tau-1}^{2} \sigma_{u_{\tau-1}}^{2}$

${ }^{43}$ See Fuller (1996, p. 492). 


$$
\begin{aligned}
=\sigma_{f_{\tau}}^{2} & +2 \sigma_{z}^{2}+2 \sigma_{z f_{\tau}}(\text { from (4)) } \\
\operatorname{Cov}\left(r_{\tau}, r_{\tau-1}\right) & =E\left(\left(\theta_{\tau-1} u_{\tau-1}+u_{\tau}\right)\left(\theta_{\tau-2} u_{\tau-2}+u_{\tau-1}\right)\right) \\
& =\theta_{\tau-1} \sigma_{u_{\tau-1}}^{2} \\
& =-\sigma_{z}^{2}-\sigma_{z f_{\tau-1}}(\text { from (5)) }
\end{aligned}
$$

Since $\theta$ and $\sigma_{u_{\tau}}^{2}$ are estimated previously in (6), $\sigma_{z_{\tau}}^{2}$ and $\sigma_{f_{\tau}}^{2}$ are determined in (9) and (8), respectively. Specifically, the noise variance measure $2 \sigma_{z}^{2}+2 \sigma_{z f_{\tau-1}}$ and the informational variance $\sigma_{f_{\tau}}^{2}$ are expressed with the variance of the moving average $\left(\sigma_{u_{\tau}}^{2}\right)$ in (6) as follows:

Noise variance measure $=2 \sigma_{z}^{2}+2 \sigma_{z f_{\tau-1}}=-2 \theta_{\tau-1} \sigma_{u_{\tau-1}}^{2}$

Informational variance ${ }^{44}=\sigma_{f_{\tau}}^{2}=\sigma_{u_{\tau}}^{2}+\theta_{\tau-1}^{2} \sigma_{u_{\tau-1}}^{2}-\left(2 \sigma_{z}^{2}+2 \sigma_{z f_{\tau-1}}\right)$

$$
=\sigma_{u_{\tau}}^{2}+\theta_{\tau-1}^{2} \sigma_{u_{\tau-1}}^{2}+2 \theta_{\tau-1} \sigma_{u_{\tau-1}}^{2}
$$

If noise does not exist, then the total variance is identical to the informational variance. Note that if $\theta_{\tau-1}$ is positive, then the variance of the random walk component $\left(\sigma_{f_{\tau}}^{2}\right)$ could be larger than the variance of the returns $\left(\operatorname{Var}\left(r_{\tau}\right)\right)$. Therefore, if the informational and noise factors influence price changes in opposite directions from one other $\left(\sigma_{z f_{\tau}}<0\right)$,

\footnotetext{
${ }^{44}$ The unconditional version of the informational variance is $(1+\theta)^{2} \sigma_{u}^{2}$. This is analogous to (A10) in Appendix 1.
} 
and hence price changes are less than expected given information alone, then the variance of the random walk component is larger than the variance of the returns. ${ }^{45}$

Whether the informational variance becomes larger or smaller than the variance of the returns, the difference is always caused by the behavior of noise traders. In other words, the absolute difference between the total variance and the informational variance is due to noise. Price changes are due to both information arrivals and liquidity-motivated trading (noise). If prices only reflect noise, then price changes are solely determined by the difference between the supply and demand of liquidity-motivated trading volume. Therefore, the variance of the current price change is the combination of the variance of information arrivals and the variance of noisy liquidity-motivated trading, which is measured by $\sigma_{u_{\tau}}^{2}+\theta_{\tau-1}^{2} \sigma_{u_{\tau-1}}^{2}$. Given the variance of the partially revealing price changes $\left(\sigma_{u_{\tau}}^{2}+\theta_{\tau-1}^{2} \sigma_{u_{\tau-1}}^{2}\right)$, which is only partially revealing because of the presence of noise, the influence of the noise factor is the absolute value of the variance of noise, namely $2\left|\theta_{\tau-1}\right| \sigma_{u_{\tau-1}}^{2}$. Consequently, the measure of price informativeness is defined as

Measure of price informativeness $=1-\frac{\mid \text { Noise variance measure } \mid}{\text { Total variance }}=1-\frac{2\left|\theta_{\tau-1}\right| \sigma_{u_{\tau-1}}^{2}}{\sigma_{u_{\tau}}^{2}+\theta_{\tau}^{2} \sigma_{u_{t-1}}^{2}}$

This measure of the informativeness of price shows that if the price change is only caused by information arrivals, then the informativeness measure equals 1 . However, as more

\footnotetext{
${ }^{45}$ Enders (2004) states the condition under which the variance of the information component is larger than the variance of the returns, as follows: "If $\left(1+\beta_{1}+\beta_{2}\right)^{2}>1$, then the trend is more volatile than $y_{t}$ since the negative correlation between $\mu_{t}$ and the stationary component act to smooth the $\left\{y_{t}\right\}$ sequence.” The term $\left(1+\beta_{1}+\beta_{2}\right)^{2}$ corresponds to $\left(1 \theta_{\tau-1}^{2} 2 \theta_{\tau-1}\right)^{\prime}$ in (11), and $y_{t}$ and $\mu_{t}$ are the observed price and the trend component of the observed price sequence, respectively.
} 
noise exists, where noise is the absolute value of $2 \sigma_{z_{\tau}}^{2}+2 \sigma_{z f_{\tau}}$, then the informativeness measure becomes smaller and (at the extreme) converges to zero when the price change is caused solely by noise. Note that $\sigma_{u_{\tau}}^{2}$ is allowed to be heteroskedatic, and therefore $\sigma_{u_{\tau}}^{2}$ and $\sigma_{r u_{\tau}}$ are modeled as autoregressive processes. I implement the mean model given in (13) and the models for the variance and the covariance given in (15) and (16) simultaneously. ${ }^{46}$

$r_{\tau}=\theta_{\tau-1} u_{\tau-1}+u_{\tau}$

$\theta_{\tau}=\frac{\sigma_{r u_{\tau}}}{\sigma_{u_{\tau}}^{2}}$

$\sigma_{u_{\tau}}^{2}=\alpha_{0}+\alpha_{1} u_{\tau-1}^{2}+\beta_{1} \sigma_{u_{\tau-1}}^{2}$

$\sigma_{r u_{\tau}}=\delta_{0}\left(1-\delta_{1}-\gamma_{1}\right)+\delta_{1} r_{\tau-1} u_{\tau-2}+\gamma_{1} \sigma_{r u_{\tau-1}}$

A quasi-maximum likelihood estimation technique is used to solve the models in (13), (14), (15) and (16), with the quasi-likelihood function in (17). ${ }^{47}$ The variance $\left(\hat{\sigma}_{u_{\tau}}^{2}\right)$ and covariance ( $\hat{\sigma}_{r u_{\tau}}$ ) of the moving average estimated by the log likelihood function in (17)

\footnotetext{
${ }^{46}$ The ARMA processes in (15) and (16) can be expanded to the orders of $\mathrm{p}$ and q.

${ }^{47}$ In (24), $\operatorname{Cov}\left(r_{\tau}, u_{\tau-1}\right)=E\left(\left(r_{\tau}-E\left(r_{\tau}\right)\right)\left(u_{\tau-1}-E\left(u_{\tau-1}\right)\right)\right)=E\left(r_{\tau} u_{\tau-1}\right)$, since the mean of the returns is zero $\left(E\left(r_{\tau}\right)=\theta_{\tau} E\left(u_{\tau-1}\right)=0\right)$. The expected covariance is modeled by an ARMA process, as shown in (24). The term $\delta_{1}$ captures innovations in the covariance between the returns and the past residuals, whereas $\gamma_{1}$ captures the stability of the process.
} 
is used to determine the contribution of information and noise to volatility, as estimated by $\sigma_{f \tau}^{2}$ in (11) and $\sigma_{z \tau}^{2}$ in (10).

$$
l=-0.5 \sum_{\tau=1}^{T N}\left(\log \left(\sigma_{u \tau}^{2}\right)+\frac{u_{\tau}^{2}}{\sigma_{u \tau}^{2}}\right)
$$

\subsubsection{Testing for price informativeness}

Testing for the existence of the noise factor on price changes is equivalent to testing whether the moving average coefficient is significantly different from zero, i.e., $\theta_{\tau-1}=0$. If the residuals in (13) are normally distributed conditional at time $\tau$, then the moving average coefficient will also be normally distributed conditional on time $\tau$. Specifically,

$\theta_{\tau-1} \sim N\left(0, \operatorname{Var}\left(\theta_{\tau-1}\right)\right)$.

The noise variance $-2 \theta_{\tau-1} \sigma_{u_{\tau-1}}^{2}$ follows from the normal distribution such that:

$$
\begin{aligned}
& -2 \theta_{\tau-1} \sigma_{u_{\tau-1}}^{2} \sim N\left(0,4 \sigma_{u_{\tau-1}}^{4} \operatorname{Var}\left(\theta_{\tau-1}\right)\right) \\
& \text { or }-2 \sigma_{u_{\tau-1}}^{2} \theta_{\tau-1} \sim-2 \sigma_{u_{\tau-1}}^{2} N\left(0, \operatorname{Var}\left(\theta_{\tau-1}\right)\right)
\end{aligned}
$$

The moving average coefficient $\left(\theta_{\tau-1}\right)$ is determined at time period $\tau$, meaning that $\theta_{\tau-1}$ varies at time $\tau$, whereas $\sigma_{u_{\tau-1}}^{2}$ is a predetermined value for time $\tau$ and therefore is invariant. It follows that testing whether $-2 \theta_{\tau-1} \sigma_{u_{\tau-1}}^{2}=0$ is equivalent to testing whether $\theta_{\tau-1}=0$. Hence, the hypothesis of a null noise factor in the price changes is specified as:

$H_{0}: \theta_{\tau-1}=0$ 
The lower and upper limit at the 95\% confidence level under the null hypothesis is ${ }^{48}$

$$
-1.96 \sqrt{\operatorname{Var}\left(\hat{\theta}_{\tau-1}\right)} \leq \hat{\theta}_{\tau-1} \leq 1.96 \sqrt{\operatorname{Var}\left(\hat{\theta}_{\tau-1}\right)}
$$

where

$$
\operatorname{Var}\left(\hat{\theta}_{\tau-1}\right)=\frac{\hat{\sigma}_{u \tau}^{2}}{\sum_{s=1}^{\tau-1} u_{s}^{2}}
$$

Since the estimates of $\hat{\sigma}_{u \tau}^{2}$ are used in (22), the student t-test is appropriate. If the null hypothesis is accepted, then the influence of noise on the change in price at time period $\tau$ is not statistically significant, i.e. the price changes are only based on information.

\subsection{Data}

Three major spot stock indexes are employed for the model of variance decomposition by information and noise: the S\&P 500 stock index, the Dow Jones Industrial Average Index, and the Nasdaq stock index. Stock indexes are chosen due to their volatile behavior, including the Nasdaq bubble in the late 1990's and the stock market crash in the early 2000's, both of which potentially reflect the behavior of noninformational noise traders. Variance decomposition by information content will show how noise factors influenced the stock indexes during these time periods.

\footnotetext{
${ }^{48}$ The variance of the conditional moving average is obtained as follows: since $\hat{\theta}_{\tau-1}=\left(u_{\tau-1}^{\prime} u_{\tau-1}\right)^{-1} u_{\tau-1}^{\prime} r_{\tau}=\theta_{\tau-1}+\left(u_{\tau-1}^{\prime} u_{\tau-1}\right)^{-1} u_{\tau-1}^{\prime} u_{\tau}$ then, $E\left[\left(\hat{\theta}_{\tau-1}-\theta_{\tau-1}\right)^{2}\right]=\left(u_{\tau-1}^{\prime} u_{\tau-1}\right)^{-1} u_{\tau-1}^{\prime} E\left[u_{\tau} u_{\tau}^{\prime}\right] u_{\tau-1}\left(u_{\tau-1}^{\prime} u_{\tau-1}\right)^{-1}=\sigma_{u \tau}^{2}\left(u_{\tau-1}^{\prime} u_{\tau-1}\right)^{-1}=\sigma_{u \tau}^{2} / \sum_{s=1}^{\tau-1} u_{s}^{2}$
} 


\subsection{Results of variance decomposition by information and noise}

Table 1 shows the estimation results for the variance decomposition by information and noise as described in (13) through (16). The results show that the covariance processes are stationary, as shown by the persistency measures of gamma1 being 0.998 , 0.997 and 0.998 for the S\&P 500, Dow Jones and Nasdaq indexes, respectively. The estimated unconditional covariances between the returns and the lagged residuals estimated by delta0 are all statistically zero. In combination with the conditional variances, the moving average coefficients are time-varying and therefore include both the informational and the noise factors.

Figures 2.1A, 2.1B and 2.1C show the estimated time-varying moving average

coefficient (theta, $\hat{\theta}_{\tau}$ ) for the three stock indexes. The results reveal an important property of the moving average coefficient. The moving average coefficient reflects the behavior of the noise trading factor on price. During the mid-1990s the noise factor is statistically significant for both the S\&P 500 and the Dow Jones indexes. This time period corresponds to when the bubble in the stock market started. Note that the moving average coefficient is significantly positive when the bubble increases in size, not when the bubble is at its maximum. An interesting result for the S\&P 500 and Dow Jones indexes is that the moving average coefficient is negative due to the effects of uninformed speculators for 2002, 2003, and 2004. This negative coefficient is consistent with stock indexes being below their true values during this time period. As shown in Figure 2.1, when the moving average coefficient increases to a value above the 
statistically significant positive band, then this is consistent with overvaluation (a potential bubble). Similarly, a negative significant coefficient represents an undervaluation or bursting of a bubble, as existed in the early 2000s. Furthermore, the index values start to decrease when the moving average coefficients turn from positive to negative.

Figures 2.2 and 2.3 show the estimated informational and noise variance components from (10) and (11) for the S\&P 500, the Dow Jones, and the Nasdaq indexes. The figures provide two new important findings. First, both the informational variance and the noise variance components are time-varying. Previous models, which decompose price into a permanent component and a stationary component, assume constant informational variance and noise variance components. Here the results show that the assumption of a constant variance for the components of the variance series is not appropriate since conditional heteroskedasticity of the time-varying informational arrivals is shown in Figures 2.2 and 2.3. Second, the measure of noise variance given in (10) reflects the changing behavior of the noise factor in relation to the information factor, where this relation is reflected in the signed moving average coefficient.

Previous studies assume that the autocovariance of the returns in (9) is only composed of $-\sigma_{z}^{2} \cdot{ }^{49}$ This restriction corresponds to the non-existence of uninformed speculators who cause the excess covariance of $\sigma_{z f_{t-1}}$, as stated in section 2.3.1. Moreover, if the uninformed speculators are ignored, then the dynamics of the measure of the noise variance are not available. The resultant restriction on $\theta_{\tau-1}$ from this assumption is that

\footnotetext{
${ }^{49}$ See Hasbrouck (1993, p. 198) for an explanation concerning the restriction of $\sigma_{z f}=0$. Models with this restriction are found in Watson (1986), Harvey (1989), Fuller (1995) and Enders (2004).
} 
$\theta_{\tau-1}$ always has to be negative. Specifically, if $\sigma_{t \tau_{\tau-1}}=0$ is assumed to be true, then $\operatorname{Cov}\left(r_{\tau}, r_{\tau-1}\right)=\theta_{\tau-1} \sigma_{u_{\tau-1}}^{2}=-\sigma_{z}^{2}$. In order for $\sigma_{z}^{2}$ to be positive then $\theta_{\tau-1}$ always must be negative. However, as the results in Figure 2.1 show, $\theta_{\tau-1}$ can possess any sign. From (10), the variance of noise per se is always positive, while the covariance between the noise factor and the informational factor can be either positive or negative, as estimated by the covariance $\sigma_{z f_{t-1}}$.

The results in Figure 2.3 show that uninformed speculators increased prices above their true values, as supported by the positive moving average values before 1998 (as shown in Figure 2.1), while the uninformed speculators caused prices to decline during 2002, 2003, and 2004, as supported by the positive moving average coefficients in the Nasdaq, S\&P 500, and Dow Jones indexes. The time-varying moving average coefficient is shown in Figures 2.1A, 2.1B and 2.1C. The noise factor is most pronounced in the Nasdaq index, since the Nasdaq results possess larger negative values of the measure of noise variance, which corresponds to a stronger negative relation between the noise and information factors, i.e., it shows that stronger uninformed speculators exist in this market. Hence, the bubble in the Nasdaq was more severe than any possible bubble in the S\&P500 or Dow Jones indexes.

Analytically, if $\sigma_{z f_{\tau-1}}<0<\sigma_{z}^{2}$ and $\left|\sigma_{z f_{\tau-1}}\right|>\sigma_{z}^{2}$, then $\theta_{\tau-1}=-\left(\sigma_{z}^{2}+\sigma_{z f_{\tau-1}}\right) / \sigma_{u_{t-1}}^{2}>0$ in (10). In other words, if the uninformed speculators cause a price to move in the same direction as the past price change $\left(u_{\tau-1}\right)$, then this uninformed speculators' effect is opposite the information content, as shown by $\sigma_{z f_{\tau-1}}<0$. Therefore, if the negative $\sigma_{z f_{\tau-1}}$ 
value continuously becomes more negative for a prolonged time period $\left(\sigma_{z f_{s}}<\sigma_{2 f_{\tau}}<0\right.$ for all $s>\tau$ ), then this should reflect a price that has deviated from its fair price. Such a result is found in Figure 2.3 for the S\&P500 and Dow Jones indexes until 1997. The $\sigma_{z f_{\tau-1}}$ for the Nasdaq is lower than for the S\&P500 and the Dow Jones for the entire sample period, signaling that the price series for the S\&P500 and Dow Jones indexes are more informative than are the prices for the Nasdaq. Note that the $\sigma_{z f_{\tau-1}}$ for the Nasdaq possessed a large positive value when the price bubble started to burst in early $2000{ }^{50}$

Figure 2.4 shows the estimated measure of price informativeness based on (12). When the moving average coefficient in Figure 2.1 reflect a pure random walk in returns then the measure of price informativeness in Figure 2.4 is equal to one. Alternatively, price informativeness is statistically less than one when the price is significantly influenced by the noise factor, such as during the 1990's for the stock market. Whereas the influence of the uninformed speculators is reflected solely by the size of the noise variance measure in (1) and Figure 2.3, the price informativeness in Figure 2.4 is affected by both information and uninformed speculators in (12), as reflected in the noise measure. In other words, even though the size of the noise variance measure is large, its influence can be smaller if the informational factor is relatively larger. The findings from Figures

\footnotetext{
${ }^{50}$ Uninformed speculators cause $\sigma_{z f_{\tau-1}}<0$, since the effect of uninformed speculators' trading on the price series is the opposite the information content. If the effects of uninformed speculators $\left(\sigma_{z f_{t-1}}<0\right)$ are significant, and therefore the difference between the efficient price and the observed price is substantial, then uninformed speculators may be able to distinguish the noise component $\left(Z_{\tau}\right)$ from the information component $\left(f_{\tau}\right)$. If uninformed speculators are able to make this distinction, and if the effect of the uninformed speculators' trading on the price suddenly disappeared, then the price would sharply revert to the efficient price, resulting in a crash of bubble (or at least a significant price change). When uninformed speculators are able to distinguish the noise component from the information component, then the excess covariance $\sigma_{z f_{\tau-1}}$ becomes zero.
} 
2.3 and 2.4 are similar. However, the measure of price informativeness is equally low for years 1995 to 1997, whereas the size of the noise measure is monotonically increasing until 1998.

Table 2.2 presents the correlation coefficients between the informational variance, the measure of the noise variance, the moving average coefficient, and the measure of price informativeness. Because the measure of the noise variance is a negative function of the moving average coefficient $\left(\theta_{\tau}\right)$, the correlation coefficient between the noise variance and the moving average coefficient is negative. Table 2.2 shows that the estimated noise variance is generally negative; when the measure of the noise variance becomes less negative then the effect of noise lessens, whereas when the noise variance is near zero then its effect becomes negligible. Since the price is more informative when the noise variance is closer to zero (rather than negative), it is not surprising that the correlation coefficient between the measure of noise variance and the measure of price informativeness is positive (since the noise variance becomes less negative as the measure of price informativeness becomes more positive). The correlation between the informational variance and the measure of informativeness is positive for all datasets, as expected. The reason why the correlation coefficients are generally above 0.5 can be explained by the significant effects of the uninformed speculators, which is measured by the non-zero correlations between the informational variance and the noise variance. When informational variance increases, there are either negative or positive reactions by the uninformed speculators since they are not able to distinguish noise from information component in price changes. As a result, the correlation coefficient between the informational variance and the measure of informativeness is positive, but the correlation 
is not necessarily large due to the correlation between the informational variance and the noise variance.

\subsection{Conclusions}

This study develops the methodology to decompose return variance into an informational component and a noise component. The model to decompose return variance given here is unique in that the decomposed informational and noisy variance components are time-varying; this decomposition allows uneven information arrivals to be captured properly. Comparatively, models by Hasbrouck (1991a, 1991b), which are similar to the Beveridge and Nelson (1981) decomposition model, do not allow the informational and the noise variance components to be time-varying. The S\&P 500 stock index, Dow Jones Industrial Average index and Nasdaq stock index are examined yearly from 1991 to 2006 to illustrate how this time-varying decomposition aids in interpreting the variance of price changes.

The results from this decomposition show that both the informational and noise variance measures are in fact time-varying, where the influence of the noise factor on the price change is reflected in the time-varying moving average coefficient. Hence, the formulation of the time-varying moving average for the variance decomposition by information and noise is important to capture the dynamics of the noise component. When the noise factor influences the price to rise above the efficient price then the moving average is statistically positive for the sample index datasets, and vise versa. Empirical results show that when the moving average coefficient is statistically positive for an extended period of time then a price bubble forms. If the moving average 
coefficient is negative, which happened from 2002 to 2004, then the stock indexes experience a floor value.

The methodology developed here to decompose the variance into information and noise provides several possible research avenues for investigating price behavior, such as the examination of price bubbles, the forecast of long-run price movements, and the market microstructure analysis of the volume-volatility relation. The analyses of the relation between the trading demand function (trading pattern), information, and noise using the informational variance and noisy variance components will be discussed in Essay 3. 


\section{Table 2.1: Daily Estimation Results of Variance Decomposition by Information}

Table 2.1 shows the estimation results for variance decomposition by information and noise in (13) through (16) for the S\&P 500, Dow Jones, and Nasdaq indexes. Specifically,

$$
\begin{aligned}
r_{\tau}= & \theta_{\tau-1} u_{\tau-1}+u_{\tau} \\
\theta_{\tau-1} & =\sigma_{r u_{\tau}} / \sigma_{u_{\tau-1}}^{2} \\
\sigma_{u_{\tau}}^{2} & =\alpha_{0}+\alpha_{1} u_{\tau-1}^{2}+\beta_{1} \sigma_{u_{\tau-1}}^{2} \\
\sigma_{r u_{\tau}} & =\delta_{0}\left(1-\delta_{1}-\delta_{2}-\gamma_{1}\right)+\delta_{1} r_{\tau-1} u_{\tau-2}+\delta_{2} r_{\tau-2} u_{\tau-3}+\gamma_{1} \sigma_{r u_{\tau-1}} \\
& =\delta_{0}\left(1-\delta_{1}-\gamma_{1}\right)+\delta_{1} r_{\tau-1} u_{\tau-2}+\gamma_{1} \sigma_{r u_{\tau-1}}
\end{aligned}
$$

$\mathrm{P}$-values for significance are in parenthesis.

\begin{tabular}{cccc}
\hline & S\&P 500 & Dow Jones & Nasdaq \\
\cline { 2 - 4 } Alpha0 & 0.000 & 0.000 & 0.000 \\
& $(0.000)$ & $(0.000)$ & $(0.000)$ \\
Alpha1 & 0.056 & 0.063 & 0.075 \\
& $(0.006)$ & $(0.000)$ & $(0.000)$ \\
Beta1 & 0.938 & 0.928 & 0.920 \\
& $(0.007)$ & $(0.000)$ & $(0.000)$ \\
Delta0 & 0.000 & 0.000 & 0.000 \\
& $(0.581)$ & $(0.551)$ & $(0.222)$ \\
Delta1 & 0.000 & 0.000 & 0.001 \\
& $(0.008)$ & $(1.000)$ & $(0.294)$ \\
Delta2 & 0.002 & 0.001 & \\
Gamma1 & $(0.858)$ & $(0.883)$ & \\
Likelihood & 0.997 & 0.998 & 0.998 \\
$\mathrm{~N}$ & $(0.002)$ & $(0.000)$ & $(0.000)$ \\
& 13,220 & 13,226 & 11,976 \\
\hline
\end{tabular}




\section{Figure 2.1A: Estimated Time-varying Moving Average Coefficient for S\&P 500}

Figures 2.1A, 2.1B, and 2.1C show the estimated time-varying coefficient $\left(\theta_{\tau}\right)$ of the moving average in (6) and (13), and their confidence limits at the 95\% confidence interval for the S\&P 500, Dow Jones, and Nasdaq indexes. The moving average coefficient is assumed to be stochastic to accommodate the timevarying informativeness found in price changes $\left(r_{\tau}\right)$. The coefficient is obtained by ARMA processes. If the estimated moving average coefficient is outside of the confidence interval at period $\tau$, then the return does not follow the random walk process during period $\tau$.

$$
\begin{aligned}
r_{\tau}= & \theta_{\tau} u_{\tau-1}+u_{\tau} \\
\theta_{\tau}= & \frac{\operatorname{Cov}_{\tau-1}\left[r_{\tau}, u_{\tau-1}\right]}{\operatorname{Var}_{\tau-1}\left[u_{\tau-1}\right]}=\frac{\sigma_{r u_{\tau}}}{\sigma_{u_{\tau-1}}^{2}} \\
\sigma_{u_{\tau}}^{2} & =\alpha_{0}+\alpha_{1} u_{\tau-1}^{2}+\beta_{1} \sigma_{u_{\tau-1}}^{2} \\
\sigma_{r u_{\tau}} & =\delta_{0}\left(1-\delta_{1}-\delta_{2}-\gamma_{1}\right)+\delta_{1} r_{\tau-1} u_{\tau-2}+\delta_{1} r_{\tau-2} u_{\tau-3}+\gamma_{1} \sigma_{r u_{\tau-1}} \\
& =\delta_{0}\left(1-\delta_{1}-\gamma_{1}\right)+\delta_{1} r_{\tau-1} u_{\tau-2}+\gamma_{1} \sigma_{r u_{\tau-1}}
\end{aligned}
$$

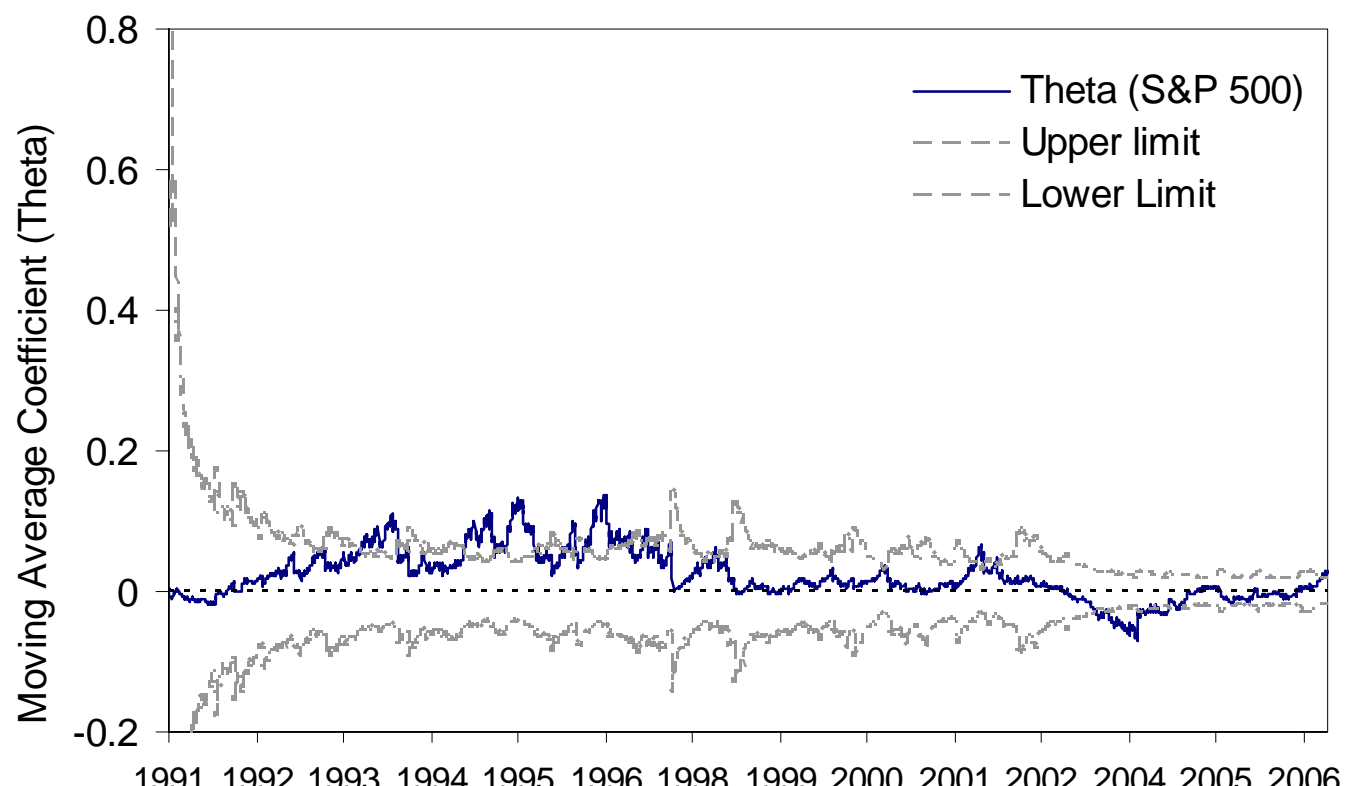


Figure 2.1B: Estimated Time-varying Moving Average Coefficient for Dow Jones

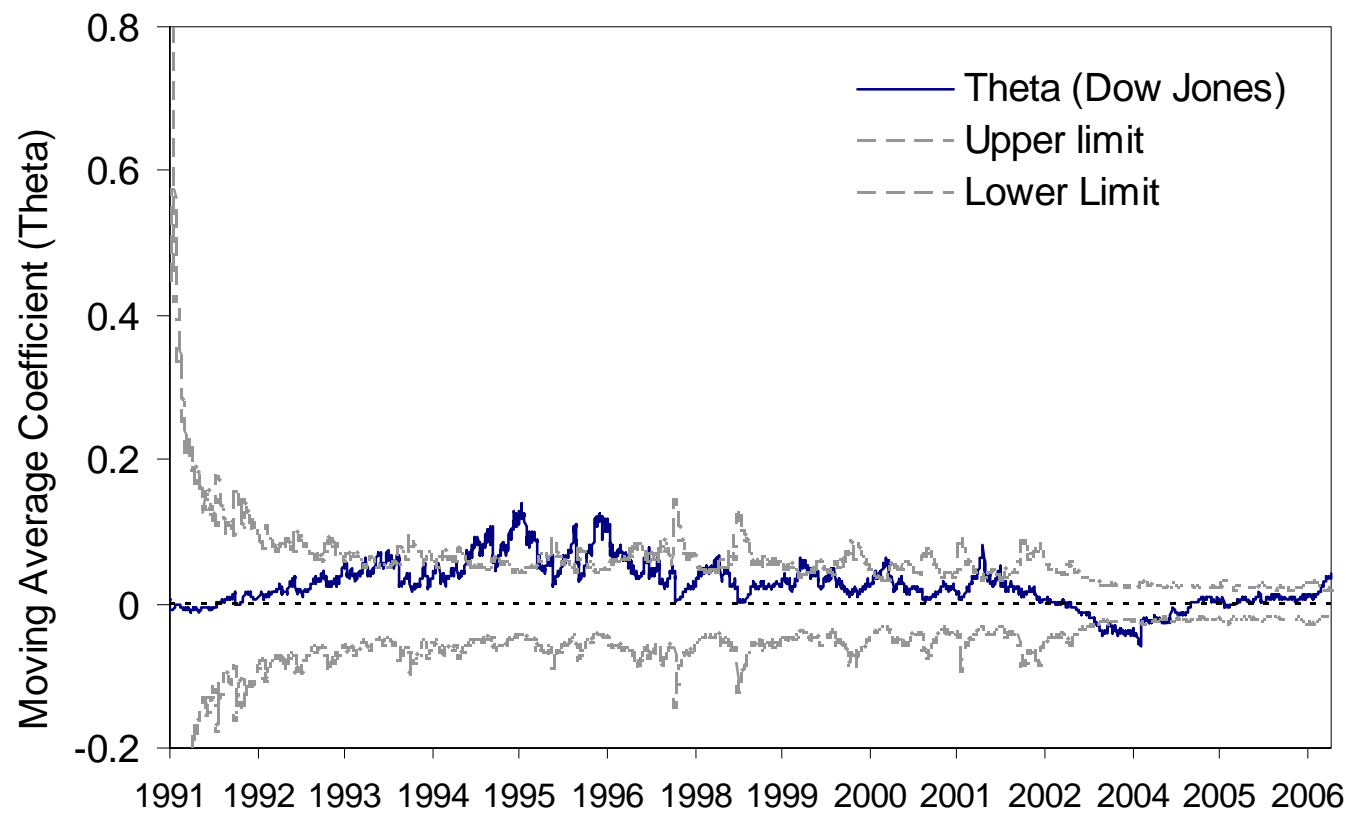

Figure 2.1C: Estimated Time-varying Moving Average Coefficient for Nasdaq

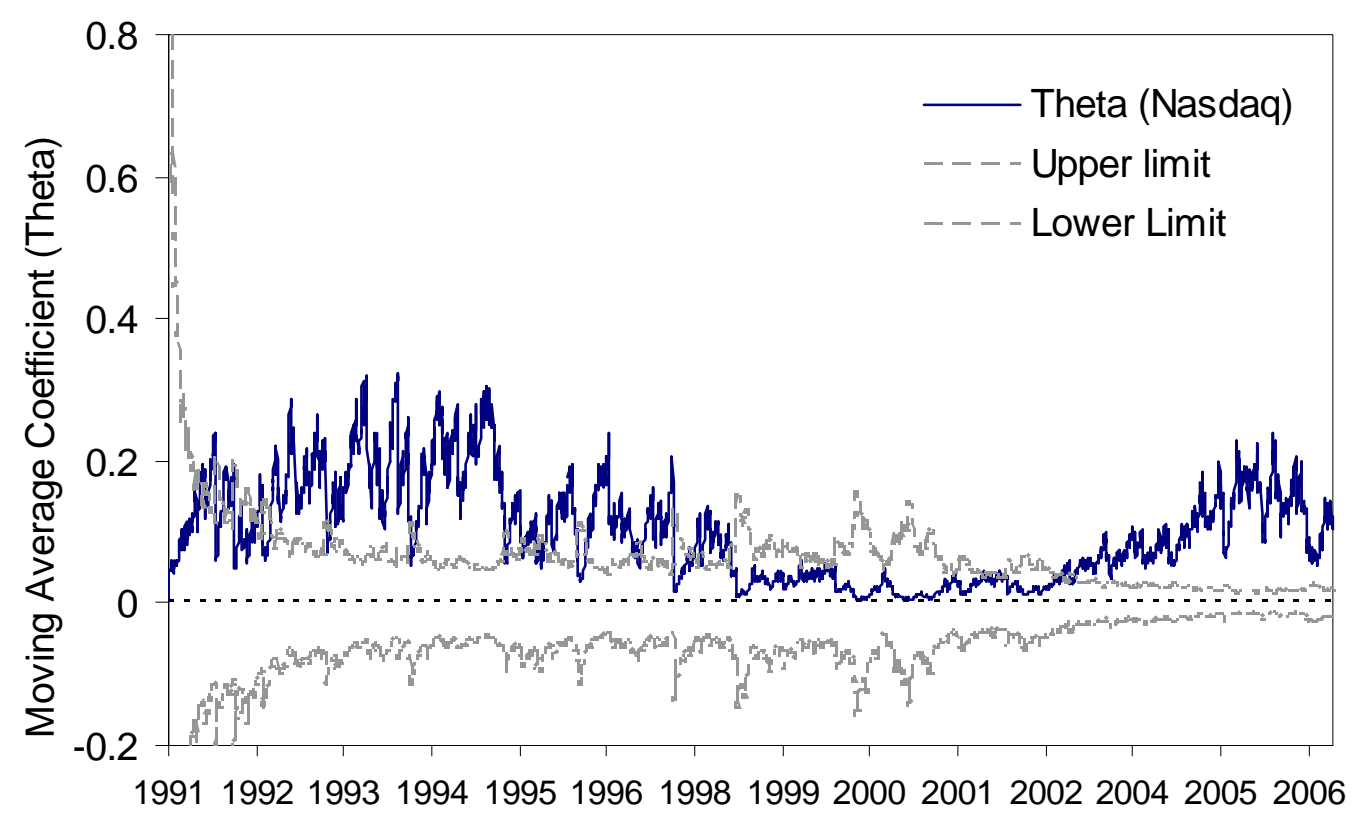




\section{Figure 2.2: Estimated Informational Variances}

Figure 2.2 shows the estimated informational variances for the S\&P 500 and Nasdaq indexes, as described in (11). The results for the Dow Jones index are indistinguishable from those of the S\&P 500. The informational variance is specified as follows:

Informational variance $=\sigma_{f_{\tau}}^{2}=\sigma_{u_{\tau}}^{2}+\theta_{\tau-1}^{2} \sigma_{u_{\tau-1}}^{2}+2 \theta_{\tau-1} \sigma_{u_{\tau-1}}^{2}$

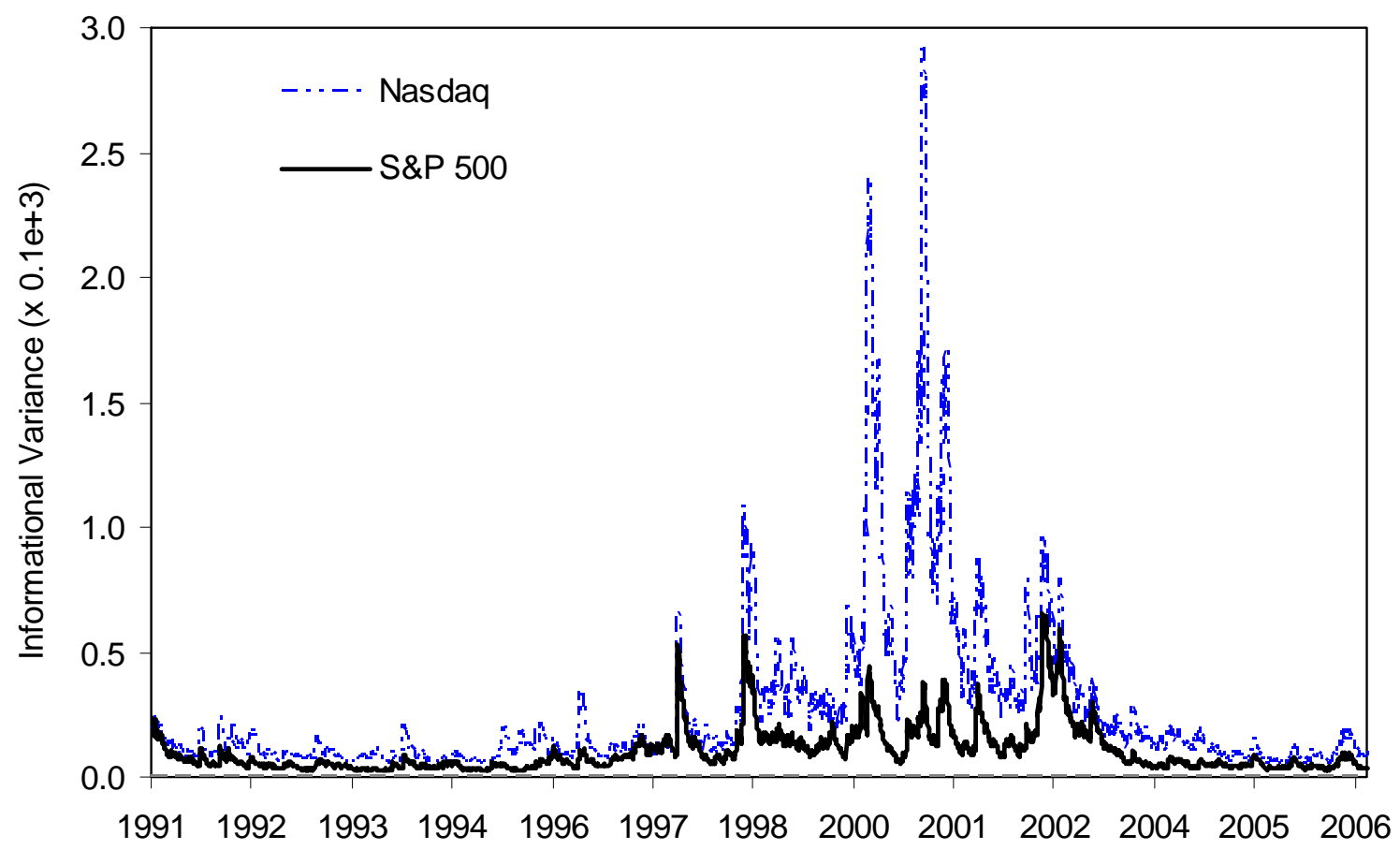




\section{Figure 2.3: Estimated Measure of Noise Variances}

Figure 2.3 shows the estimated measure of the noise variance for the S\&P 500, Dow Jones, and Nasdaq indexes, as described in (10). Note that the noise variance measure includes the covariance and hence can be negative. When the measure of noise has a value of zero then noise is not a factor that prevents price from revealing information. The measure of noise variance is specified as follows:

Noise variance measure $=2 \sigma_{z}^{2}+2 \sigma_{z f_{\tau-1}}=-2 \theta_{\tau-1} \sigma_{u_{\tau-1}}^{2}$

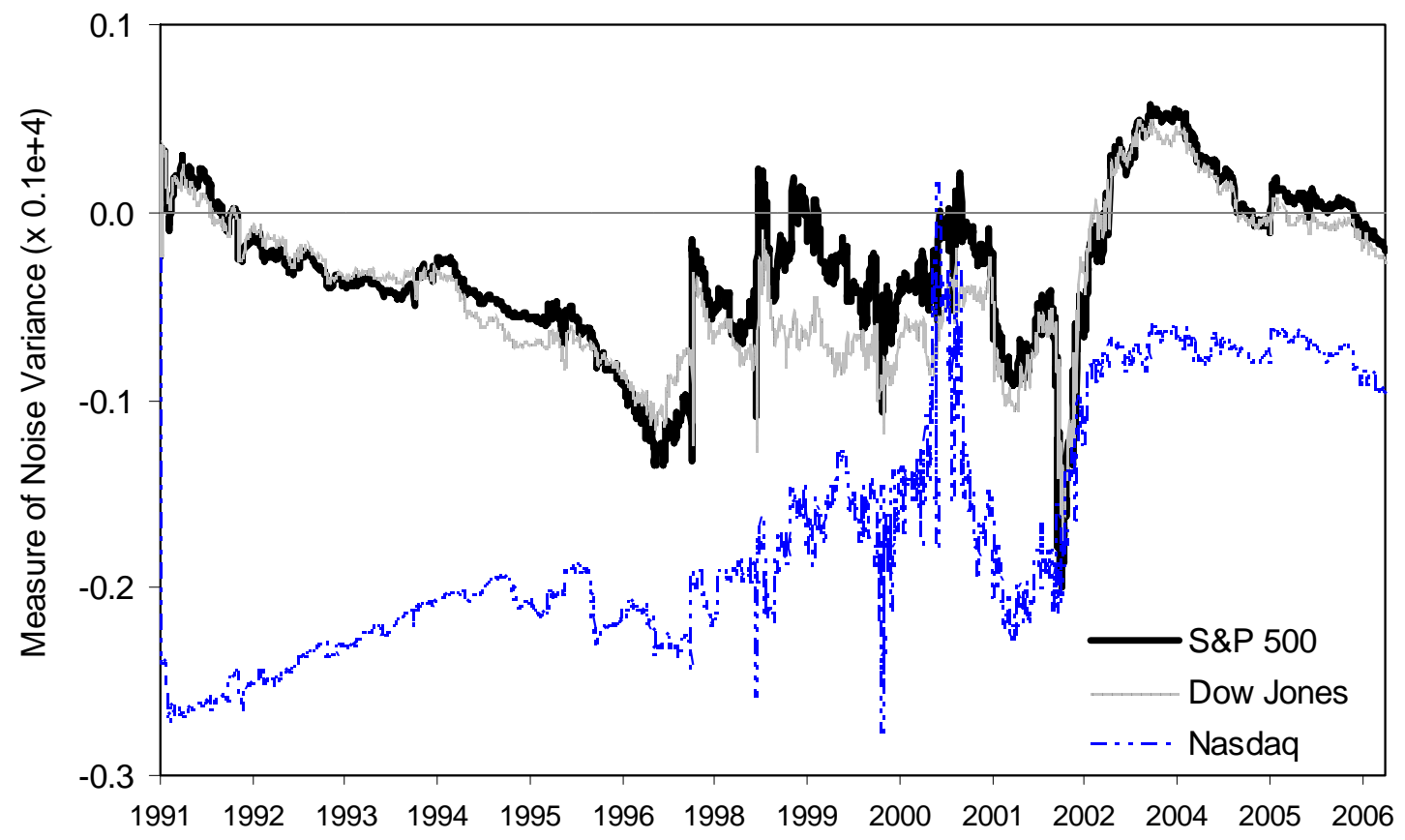




\section{Figure 2.4: Estimated Price Informativeness}

Figure 2.4 shows the estimated time-varying measure of price informativeness for the S\&P 500 and Nasdaq indexes, as given by (12). The estimated time-varying measure of price informativeness for the Dow Jones index is indistinguishable from that of the S\&P 500. The measure of price informativeness equals one if there is no noise in price such that price will fully reveal information. The measure of price informativeness is specified as follows:

Measure of price informativeness $=1-\frac{\mid \text { Noise variance measure } \mid}{\text { Total variance }}=1-\frac{2\left|\theta_{\tau-1}\right| \sigma_{u_{\tau-1}}^{2}}{\sigma_{u_{\tau}}^{2}+\theta_{\tau-1}^{2} \sigma_{u_{\tau-1}}^{2}}$

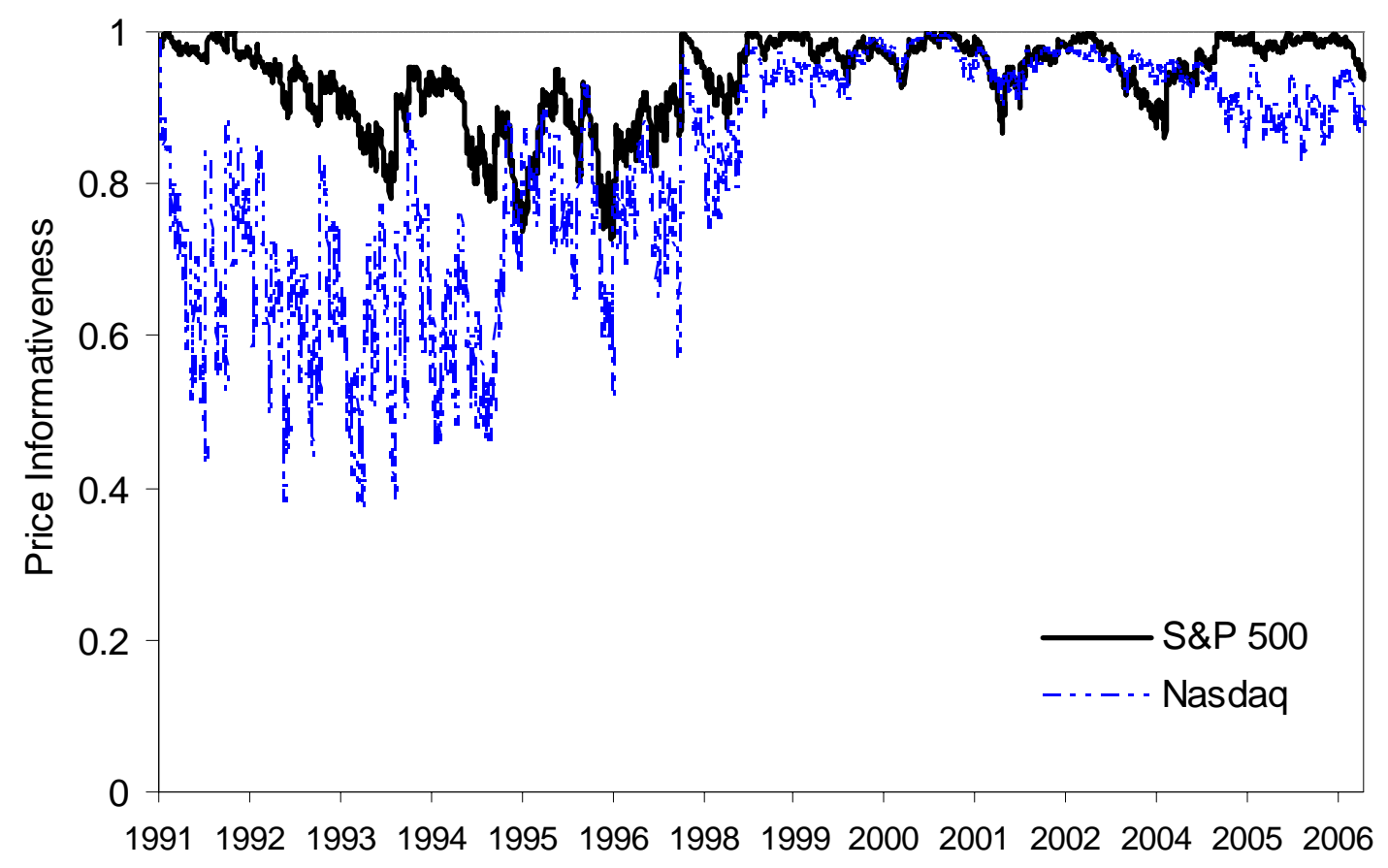


Table 2.2: Correlation Coefficients between Variance Components Decomposed into Information and Noise

Table 2.2 shows the Pearson correlation coefficients between the informational and noise variance components, the measures of informativeness, and the moving average coefficients for the S\&P 500, Dow Jones, and Nasdaq indexes. The total number of observations is 3,974 for the computations of the correlation coefficients for all datasets. The numbers in the parentheses represent the $\mathrm{p}$-values for significance given the null hypothesis of zero correlation.

Panel A: S\&P 500 Index

\begin{tabular}{lccc}
\hline & Noise Variance & Informativeness & Theta \\
\cline { 2 - 4 } Informational Variance & -0.261 & 0.409 & -0.224 \\
& $(0.000)$ & $(0.000)$ & $(0.000)$ \\
Noise Variance & & 0.441 & -0.712 \\
& & $(0.000)$ & $(0.000)$ \\
Informativeness & & -0.811 \\
& & & $(0.000)$ \\
\hline
\end{tabular}

Panel B: Dow Jones Industrial Average Index

\begin{tabular}{lccc}
\hline & Noise Variance & Informativeness & Theta \\
\cline { 2 - 4 } Informational Variance & -0.339 & 0.334 & -0.184 \\
& $(0.000)$ & $(0.000)$ & $(0.000)$ \\
Noise Variance & & 0.465 & -0.709 \\
& & $(0.000)$ & $(0.000)$ \\
Informativeness & & -0.829 \\
& & & $(0.000)$ \\
\hline
\end{tabular}

Panel C: Nasdaq Index

\begin{tabular}{lccc}
\hline \multirow{3}{*}{ Informational Variance } & Noise Variance & Informativeness & Theta \\
\cline { 2 - 4 } & 0.175 & 0.502 & -0.490 \\
Noise Variance & $(0.000)$ & $(0.000)$ & $(0.000)$ \\
& & 0.623 & -0.615 \\
Informativeness & & $(0.000)$ & $(0.000)$ \\
& & & -0.989 \\
& & & $(0.000)$ \\
\hline
\end{tabular}




\section{REFERENCES}

Admati, Anat R., 1985, A Noisy Rational Expectations Equilibrium for Multi-Asset Securities Markets, Econometrica 53, 629 - 658.

Andersen, Torben G. and Tim Bollerslev, 1997a, Intraday Periodicity and Volatility Persistence in Financial Markets, Journal of Empirical Finance 4, 115 - 158.

Andersen, Torben G. and Tim Bollerslev, 1997b, Heterogeneous Information Arrivals and Return Volatility Dynamics: Uncovering the Long-Run in High Frequency Returns, Journal of Finance 52, 975 - 1005.

Bera, Anil K., Matthew L. Higgins, and Sangkyu Lee, 1992, Interaction Between Autocorrelation and Conditional Heteroscedasticity: A Random-Coefficient Approach, Journal of Business and Economic Statistics 10, 133 - 142.

Beveridge, Stephen and and Charlese Nelson, 1981, A New Approach to Decomposition of Economic Time Series into Permanent and Transitory Components with Particular Attention to Meausurement of the Business Cycle, Journal of Monetary Economics 7, $151-174$.

Enders, Walter, 2004, Applied Econometric Time Series, $2^{\text {nd }}$ Ed.

Engle, Robert, F., Magdalena E. Sokalska and Ananda Chanda, 2006, Forecasting intraday volatility in the US equity market - multiplicative component GARCH, Working paper, UCSD.

Fama, Eugene. F., 1970, Efficient Capital markets: A Review of Theory and Empirical Work, Journal of Finance 25, 28 - 30.

Fuller, Wayne A., 1995, Introduction to Statistical Time Series, $2^{\text {nd }}$ Ed.

Harvey, Andrew C., 1989, Forecasting, Structural Time Series Models and the Kalman Filter

Hasbrouck, Joel, 1991a, Measuring the Information Content of Stock Trades, Journal of Finance 46, 179 - 207.

Hasbrouck, Joel, 1991b, The Summary Informativeness of Stock Trades: An Econometric Analysis, Review of Financial Studies 4, 571 - 595.

Hasbrouck, Joel, 1993, Assessing the Quality of a Security Market: A New Approach to Transaction-Cost Measurement, Review of Financial Studies 6, 191 - 212. 
Hasbrouck, Joel, 1995, One Security, Many Markets: Determining the Contributions to Price Discovery, Journal of Finance 50, 1175 - 1199.

Hasbrouck, Joel, 2002, Stalking the "efficient price" in market microstructure specifications: an overview, Journal of Financial Markets 5, 329 - 339.

Kim, Oliver, and Robert E. Verrecchia, 1991, Trading Volume and Price Reactions to Public Announcements, Journal of Accounting Research 29, 302 - 321

Shalen, Chatherine, T., 1993, Volume, Volatility, and the Dispersion of Beliefs, Review of Financial Studies 6, 405 - 434.

Lo, Andrew and A. Craig MacKinlay, 1988, Stock Market Prices do not follow Random Walk: Evidence from a simple specification Test, Review of Financial Studies 1, 41 66.

Watson, Mark W., Univariate Detrending Methods with Stochastic Trends, Journal of Monetary Economics 18, 49 - 75. 


\section{Appendix 2.1: The variance of the random-walk component of observed price}

This appendix explains the derivation of the variance of the random walk component (or the informational variance), a concept discussed in Hasbrouck (1991a, 1991b, 1995). The observed price is the sum of random walk and transitory components that reflects various microstructure effects, as follows:

$p_{t}=m_{t}+s_{t}$

where $m_{t}$ is an integrated process (a unit process) that represents a random walk, as follows:

$m_{t}=m_{t-1}+u_{t}$

where $E\left[u_{t}\right]=0 ; E\left[u_{t}^{2}\right]=\sigma_{u}^{2} ; E\left[u_{t} u_{s}\right]=0$ for $t \neq s$. From (A1) and (A2), the first difference of price is obtained as

$\Delta p_{t}=p_{t}-p_{t-1}=m_{t}-m_{t-1}+s_{t}-s_{t-1}=u_{t}+s_{t}-s_{t-1}$

where $\Delta p_{t}$ has a stochastic component $\left(u_{t}+s_{t}-s_{t-1}\right)$ which is covariance stationary. Hence, the Wold representation guarantees that the covariance stationary process $\Delta p_{t}$ can be represented by an infinite moving average of an uncorrelated mean zero process with a constant variance $\left(0, \sigma^{2}\right)$.

$\Delta p_{t}=\varepsilon_{t}+\psi_{1} \varepsilon_{t-1}+\psi_{2} \varepsilon_{t-2}+\cdots$

where $E\left[\varepsilon_{t}\right]=0$ and $E\left[\varepsilon_{t} \varepsilon_{s}\right]=0$ for $t \neq s$. Using the lag operator $(L), L p_{t}=p_{t-1}, \Delta p_{t}$ is rewritten as follows;

$\Delta p_{t}=p_{t}-p_{t-1}=p_{t}-L p_{t}=(1-L) p_{t}$

and by (4),

$$
\begin{aligned}
\Delta p_{t} & =\varepsilon_{t}+\psi_{1} \varepsilon_{t-1}+\psi_{2} \varepsilon_{t-2}+\cdots \\
& =\varepsilon_{t}+\psi_{1} L \varepsilon_{t}+\psi_{2} L^{2} \varepsilon_{t}+\psi_{3} L^{3} \varepsilon_{t}+\cdots \\
& =\left(1+\psi_{1} L+\psi_{2} L^{2}+\psi_{3} L^{3}+\cdots\right) \varepsilon_{t} \\
& =\psi(L) \varepsilon_{t}
\end{aligned}
$$

In short, $\Delta p_{t}=(1-L) p_{t}=\psi(L) \varepsilon_{t}$. 
The equation (A2) can be rewritten as

$m_{t}-m_{t-1}=u_{t}$

or

$m_{t}-L m_{t}=(1-L) m_{t}=u_{t}$

If include $(1-L)$ for both sides of (A1), then

$(1-L) p_{t}=(1-L) m_{t}+(1-L) s_{t}$

By substituting (A5) and (A6) into (A7),

$\psi(L) \varepsilon_{t}=u_{t}+(1-L) s_{t}$

The above equation (A8) holds for any $L$. Hence by letting $L=1$,

$$
\begin{aligned}
\psi(1) \varepsilon_{t} & =u_{t}+(1-1) s_{t} \\
& =u_{t}
\end{aligned}
$$

By taking the variance operator, the variance of random walk component is derived as follows:

$|\psi(1)|^{2} \operatorname{Var}\left[\varepsilon_{t}\right]=\operatorname{Var}\left[u_{t}\right]$

or

$\operatorname{Var}\left[u_{t}\right]=\sigma_{u}^{2}=|\psi(1)|^{2} \sigma_{\varepsilon}^{2}$

Since $\psi(L)=\left(1+\psi_{1} L+\psi_{2} L^{2}+\psi_{3} L^{3}+\cdots\right)$, the coefficient of permanent component of the returns is $\psi(1)=\left(1+\psi_{1}+\psi_{2}+\psi_{3}+\cdots\right)$. Hence

$|\psi(1)|^{2}=\left(1+\psi_{1}+\psi_{2}+\psi_{3}+\cdots\right)^{2}$

The equation (A10) is therefore rewritten as follows:

$\sigma_{u}^{2}=\left(1+\psi_{1}+\psi_{2}+\psi_{3}+\cdots\right)^{2} \sigma_{\varepsilon}^{2}$ 
The following part of this appendix shows how to compute the share of trades in the informational variance of price, drawing on Hasbrouck (1991b). The price in (A1) is the log value in the following formulation.

$$
\begin{aligned}
& r_{t}=\sum_{i=1}^{\infty} \alpha_{i} r_{t-i}+\sum_{i=1}^{\infty} \beta_{i} x_{t-i}+\varepsilon_{1 t} \\
& x_{t}=\sum_{i=1}^{\infty} \gamma_{i} x_{t-i}+\sum_{i=1}^{\infty} \delta_{i} r_{t-i}+\varepsilon_{2 t}
\end{aligned}
$$

Where $r_{t}$ is the rate of return, $x_{t}$ is the signed transaction volume, $\operatorname{Var}\left(\varepsilon_{1 t}\right)=\sigma_{1}^{2}$, $\operatorname{Var}\left(\varepsilon_{2 t}\right)=\Omega$, and $E\left(\varepsilon_{1 t} \varepsilon_{2 t}\right)=0$. The moving average representation follows:

$$
\begin{aligned}
& r_{t}=\varepsilon_{1 t}+a_{1} \varepsilon_{1 t-1}+a_{2} \varepsilon_{1 t-2}+\cdots+b_{1} \varepsilon_{2 t-1}+b_{1} \varepsilon_{2 t-1}+b_{2} \varepsilon_{2 t-2}+\cdots \\
& x_{t}=c_{1} \varepsilon_{1 t-1}+c_{2} \varepsilon_{1 t-2}+\cdots+\varepsilon_{2 t}+d_{1} \varepsilon_{2 t-1}+d_{2} \varepsilon_{2 t-2}+\cdots
\end{aligned}
$$

where return moving average can be written using lag operator as follows:

$$
r_{t}=\left(\begin{array}{ll}
a(L) & b(L)
\end{array}\right)\left(\begin{array}{l}
\varepsilon_{1 t} \\
\varepsilon_{2 t}
\end{array}\right)
$$

and where $a(L)=1+a_{1} L+a_{2} L^{2}+\cdots$ and $b(L)=1+b_{1} L+b_{2} L^{2}+\cdots$. The variance is

$$
\begin{aligned}
& \operatorname{Var}\left[r_{t}\right]=E\left[r_{t} r_{t}^{\prime}\right] \\
& =E\left[\left(\begin{array}{ll}
a(L) & b(L)
\end{array}\right)\left(\begin{array}{l}
\varepsilon_{1 t} \\
\varepsilon_{2 t}
\end{array}\right)\left(\begin{array}{ll}
\varepsilon_{1 t} & \varepsilon_{2 t}
\end{array}\right)\left(\begin{array}{l}
a(L) \\
b(L)
\end{array}\right)\right] \\
& =\left(\begin{array}{ll}
a(L) & b(L)
\end{array}\right)\left(\begin{array}{cc}
\sigma_{1}^{2} & 0 \\
0 & \Omega
\end{array}\right)\left(\begin{array}{l}
a(L) \\
b(L)
\end{array}\right) \\
& =a(L) \sigma_{1}^{2} a(L)+b(L) \Omega b(L)
\end{aligned}
$$

Analogous to (A10), the variance of the random walk component of the price change $\left(r_{t}\right)$ is

$$
\sigma_{u}^{2}=a(1) \sigma_{1}^{2} a(1)+b(1) \Omega b(1)
$$

where $a(1)=1+a_{1}+a_{2}+\cdots$ and $b(1)=1+b_{1}+b_{2}+\cdots$. 
The portion of trades in the variance of the random walk component of price is $\sigma_{r u} / \sigma_{u}^{2}$, where $\sigma_{u x}=b(1) \Omega b(1)$. As Hasbrouck (1991b) points out, in practice the order of the moving average is restricted to be finite. 


\section{Essay 3}

\section{Intraday Tests of the Noisy Rational Expectations Information Model}

\subsection{Introduction}

This study investigates whether the proposition from the noisy rational expectations model that trading volume has an ex-ante relation to volatility (price changes) is valid when intraday data with volume by different groups of traders are analyzed. In the noisy rational expectations models of Diamond and Verrecchia (1981), Admati (1985), Kim and Verrecchia (1991), and Shalen (1993), traders’ reactions to price changes are decomposed into the components of unexpected information content and the effect of uninformed noisy traders' reactions to market activity. Uninformed traders react both to the price changes caused by information arrivals and to the price changes caused by noise (liquidity demand), since they are uninformed and cannot distinguish between these factors. Consequently, uninformed traders’ reactions to price changes are greater than those of informed traders.

Prior empirical findings using daily data support the proposition of the noisy rational expectations model that uninformed traders overreact to price changes (Daigler and Wiley, 1999; Chang, Chou, and Nelling, 2000; Wang 2002). The analysis here differs from prior studies in two ways. First, intraday data are employed rather than daily or weekly data. In fact, it is well known that the impact of new information disappears within a short period of time, suggesting that an intraday analysis is most appropriate. ${ }^{51}$ Second, a new approach (see Essay 1) is employed to remove the U-shaped volatility

\footnotetext{
${ }^{51}$ Patel and Wolfson (1984) and Ederington and Lee (1993) find that volatility remains high only for several hours after important news is recognized by the markets.
} 
seasonality effect from the intraday data before the analysis is completed. Furthermore, the intraday volume series is also adjusted for the seasonal component. ${ }^{52}$ Thus, I first capture the seasonal component by using the autoregressive filtering process developed in Essay 1 and Appendix 1 for the variances of the returns and volumes, respectively. The interday variance components of the returns then are adjusted to examine only the intraday information components. Hence, this study examines only the short-run informativenss for different type of traders.

Section 3.2 describes the literature on the noisy rational expectations model and the associated relation between trading volume and price variability. In addition, null hypotheses to examine the informativeness of the intraday data volatility series are constructed. Section 3.3 introduces three econometric models for the variance decompositions by frequency (where volatility is decomposed on interday and intraday scales) and by information arrivals and tests of the resultant null hypotheses. In section 3.4, the data for this study are briefly described. Results from the three models introduced in section 3.3 are discussed in section 3.5. The conclusions follow in section 3.6.

\subsection{Concepts and literature review}

A typical rational expectations model, such as the standard capital asset pricing model (Sharpe, 1964; Lintner, 1965; and Mossin, 1966) and the model by Grossman (1976), assume no noise, and therefore price aggregates and reveals all public and private information. Thus, if liquidity-motivated trading volume does not affect the price, then price fully reveals information and the total variance of the returns is only composed of

\footnotetext{
${ }^{52}$ Appendix 1 detains on the procedure to filter the intraday seasonal volume component.
} 
informational components. Only information-motivated trading volumes will cause price to change to reflect new information arrivals, although noise traders can also affect price changes. These models do not provide any ex-ante relation between price variability and volume, since price is a sufficient statistic and therefore any level of trading volume is consistent with the current price level. Hence, the CAPM, as well as Grossman's pricing model, do not involve including transaction volume as part of the information set. However, as Grossman (1976) points out, if the total supply (endowment) of the asset is not a known value but rather random value, then price is a function of the randomness of the total supply of the asset as well as a function of price information. Therefore, the noise in price becomes larger as the total supply of the asset becomes more random.

On the other hand, noisy rational expectations models allow the total supply of an asset to be random, and therefore this model provides an ex-ante relation between price variability and volume. ${ }^{53}$ Diamond and Verrecchia develop the first noisy rational expectations model in which the price only partially reveals information because the price reflects noise as well as information. In Diamond and Verrecchia's model the price of the asset is inversely related to the random supply, and the sensitivity of price to the random supply increases as noise (the variance of the random supply) increases; thus, the risk premium compensation for uncertainty increases as noise increases. Kim and Verrecchia (1991) analyze the reactions of price and trading volume to public announcements using the aforementioned noisy rational expectations models. They find that trading volume is positively related to price variability, with a multiplier of the weighted average beliefs in

\footnotetext{
${ }^{53}$ In noisy rational expectations models the noise that prevents price from revealing aggregated information is defined as either noisy trading volume or the non-zero variance of the noisy trading volume (Grossman, 1976; Diamond and Verrecchia, 1981).
} 
price, where price variability is a function of surprise and random supply, where surprise is defined as the unexpected portion of a new public announcement. Thus, the model concludes that the positive relation between volume and volatility becomes stronger as traders are more heterogeneous. Shalen (1993) applies the noisy rational expectations model to futures markets in which traders are divided into speculators and hedgers. Since trades for hedges are considered to be random (they are assumed to be liquidity motivated), trading by hedgers introduce noise (randomness) into the market clearing condition. Thus, the equilibrium price from the market clearing condition becomes noisy, since it includes the noise of the hedgers' trading volume. For this reason, speculators are confused as to whether the resultant price changes are caused by private information or by the liquidity trading of hedgers. Consequently, speculators overreact to price changes, since they assume that price changes from both noise trades and informed traders are based on new information. Shalen's (1993) propositions on the relation between price changes (volatility) and volume is consistent with those of Kim and Verrecchia (1991) in which volume is a function of surprise and noise.

Daigler and Wiley (1999) apply the noisy rational expectations model in Shalen (1999) to daily futures volume and volatility data by type of trader. A key aspect of their analysis is that floor traders are informed about order flow, allowing them to observe any effects of trading volume by uninformed traders to price changes caused by liquidity demand. Consequently, the reaction of informed floor traders to trading activity is expected to be more correlated to price changes caused by information than those caused 
by noise. ${ }^{54}$ Daigler and Wiley find that the positive relation between volatility and volume is driven by the general public, who are not on the floor and therefore they cannot distinguish what price changes are due to information arrivals and what price changes are due to noise from liquidity demand; the reaction of institutional traders' trading volume to price variability is mixed but often is negative in nature. ${ }^{55}$ Chang, Chou, and Nelling (2000) find that hedgers' open interest is positively related to unexpected volatility, and of the open interest of speculators is not related to expected volatility and is weakly positively related to unexpected volatility. Wang (2002) also finds that volatility covaries negatively with speculators' trading demand and positively with hedgers' trading demand, where demand is defined as the long open interest less the short open interest.

The common empirical findings on the relation between trading volume and price variability in prior studies show that noisy traders' (such as hedgers) trading volume is positively related to price variability and that the relation between informed traders' volume and price variability is mixed. Therefore, empirical findings support the proposition in the noisy rational expectations model that uninformed traders cause excessive price changes, since they react both to the price changes caused by information arrivals and to those caused by noise traders ${ }^{56}$ However, the empirical findings on the

\footnotetext{
${ }^{54}$ By definition, noisy traders do not have an ex-ante trading schedule regarding price changes, since their trades are random. Speculators have an ex-ante trading volume schedule since they are interested in profiting from information. Based on the how informed they are, speculators are classified into informed and uninformed traders. The uninformed traders over-react to price changes relative to (the better) informed traders, since they cannot distinguish noise from information. Hence, uninformed traders create an overreaction to price changes, but they are not noise creators in the classic sense of noise trading.

${ }^{55}$ Institutional traders are associated with positive relations in municipal bonds and Treasury note contracts, a negative relation in the Major Market stock index and Treasury bonds, and no significant relation for silver contracts.

${ }^{56}$ See p. 420 in Shalen (1993).
} 
relation between informed traders' volume and price changes are not consistent, and there are no clear explanations for this inconsistency. The relation between the informed traders' volume and price variability has to be understood in two distinct situations, depending on whether unexpected information is present. Under the absence of unexpected information, if the price deviates significantly from the true value by the actions of noisy traders then the informed traders will make the price converge to the true value and consequently the trades will possess a negative relation to the price changes. However, if the prices changes are a result of unexpected information then the price changes associated with the informed traders' actions will be positively related to the price changes. ${ }^{57}$

\subsection{Hypotheses and empirical methodologies}

\subsubsection{Hypotheses}

Before stating the hypotheses concerning information, noise, and trading patterns, let us examine the properties of the information-motivated traders' demand function to purchase the asset. ${ }^{58}$ Note that the demand function of information-motivated traders possesses the following two main properties: (a) A positive relation between the current

\footnotetext{
${ }^{57}$ Kim and Verrecchia (1991) decompose the positive volume reaction to price changes into the components of unexpected information content and of uninformed traders' reactions to liquidity demand. Hence, Kim and Verrecchia's model shows that informed trading volume will be positively related to the price changes created by unexpected information contents. Hasbrouck (1991b) develops an econometric tool to estimate the degree of "informativeness" of trades. Informativeness is defined as the share of trading volume in the variance of efficient price. Payne (2003) finds that foreign exchange dealers' trades contribute $41 \%$ of the efficient price volatility.

${ }^{58}$ In this study the terms demand schedule, demand function, and trading pattern are used interchangeably.
} 
demand and the expected return, (b) A negative relation between the current demand and the contemporaneous return, i.e. the expected return decreases as the contemporaneous price (return) increases. Property (a) means that information-motivated traders buy (sell) before a price increase (decrease). Consequently, if the current price $p_{\tau} \equiv \log \left(P_{\tau}\right)$ increases then the contemporaneous return $p_{\tau}-p_{\tau-1}$ increases, whereas the expected future return $E_{\tau}\left(\tilde{p}_{\tau+1}\right)-p_{\tau}$ decreases in an informationally efficient market. As a result, the current demand decreases. Hence, as stated in property (b), there is a negative relation between the contemporaneous return and the current demand. ${ }^{59}$ Therefore, if a trader demand function includes both properties (a) and (b), then the trader is an informationmotivated trader and their trades will be profitable. The detailed properties of the information-motivated traders’ demand function are analyzed in appendix 3.1 by using the typical two-period noisy rational expectations model formulation.

The noisy rational expectations models, especially Shalen (1993), explain expected returns and noise in terms of the following concepts:

a. Informed traders' expected returns are less correlated with current noise than are the expected returns of uninformed general public traders. In Shalen (1993), the correlation between expected returns and noise is caused by uninformed speculators who are not able to distinguish noise from the information component in price changes. The excess covariance between the expected returns and current noise causes the dispersion of the volatility of the

\footnotetext{
59 This study defines the property (b) of the information-motivated demand schedule as the contrarian trading pattern for the contemporaneous return.
} 
contemporaneous returns, since the current demand for the asset changes based on both the expected return and the contemporaneous return.

b. The informed trader's current demand is negatively related to the increase in both contemporaneous noise and information arrivals (see appendix 3.1). This relation directly follows from property (b) of the information-motivated traders, since informed traders will sell the asset if the current return increases, whether the increase in the current return is caused by noise or by information.

c. Informed traders' current demand is more negatively related to the increase in the current returns caused by noise than to that the return innovations caused by information. This relation occurs because informed traders can distinguish between noise and information.

Accordingly, four null hypotheses are tested as follows:

$\mathrm{H}_{1}$ : Trading volume reactions to contemporaneous price changes are independent of time-varying information arrivals and noise.

$\mathrm{HO}_{2}$ : Trading volume reactions to contemporaneous price changes caused by information are not significantly different from those caused by noise.

$\mathrm{HO}_{3}$ : Trading volume reactions of the floor traders to contemporaneous price changes caused by noise are not significantly different from the trading volume reactions of the general public.

$\mathrm{HO}_{4}$ : Trading volume reactions of floor traders to contemporaneous price changes caused by information are not significantly different from the trading volume reactions of the general public. 
If the propositions of the relations between trading volume and price variability in the noisy rational expectations model are valid, then all of the above hypotheses will be rejected.

Noisy rational expectations models do not explicitly consider information factors based on the direction (trend) of the short-term price change. Rather, information factors are described as the components of the price behavior which follow a martingale process (equivalently the random-walk process). As a result, price movements based on old information are considered noise, where the noise is irrelevant to the expected return in an informationally efficient market. In particular, if returns are computed on an interday basis (for example, daily returns are employed), then information should be fully reflected into the daily return when the information arrives.

On the other hand, if the returns are computed using high frequency intraday data, then information may not be fully reflected within a short period of time. In this case, intraday returns can be positively auto-correlated, due to a sequence of information arrivals. Floor traders will then see a change in the short-term direction of price which is caused by the autocorrelation of information arrivals. In addition, these floor traders also may observe a short-term directional price change caused by non-information factors, such as the autocorrelation caused by liquidity-motivated trading, the bid-ask spread bounce, uninformed feedback trading, etc. This study defines knowledge about the autocorrelation of price caused by either information or non-information factors as order flow information. Since this study (Essay 3) uses fifteen-minute return intervals, it is necessary to distinguish order flow information from non-order flow information. This distinction is necessary because if traders are informed of order flow then their demand 
will be positively correlated to the factors causing the autocorrelated patterns. Hence, by decomposing order flow information, the demand function of the informed traders is more properly identified. Formally, this study defines the order flow information as follows: if the current and past returns are significantly positively correlated to the future returns, then current and past returns are said to have order flow information for future returns. Therefore, it is expected that floor traders' current demand has a positive relation to the order flow component of the contemporaneous returns. The general public traders (the off-the-floor traders) are not expected to show a positive relation since they do not have information on order flow. The associated null hypothesis is as follows:

$\mathrm{H}_{5}$ : Trading volume reactions of floor traders to the order flow component of contemporaneous returns is not significantly different from the trading volume reactions of the general public.

\subsubsection{Empirical models for tests of the noisy rational expectations models}

The equations given below are used to test the null hypotheses concerning the relation between price changes and trading volume suggested by the noisy rational expectations models explained in section 3.3.1.

$$
r_{\tau}=\alpha_{0}+\alpha_{1} r_{\tau-1}+\beta_{1 \tau} \mathrm{NetVol}_{1 \tau}+\beta_{2 \tau} \mathrm{NetVol}_{2 \tau}+\beta_{3 \tau} \mathrm{NetVol}_{3 \tau}+\beta_{4 \tau} \mathrm{NetVol}_{4 \tau}+\varepsilon_{\tau}
$$

where $r_{\tau}$ is the non-seasonal intraday contemporaneous returns which are also adjusted for the interday variance components; $\mathrm{NetVol}_{k \tau}$ is the net trading volume which is defined as the total buy volume less the total sales volume of CTI $k$ at period of $\tau$. The 
coefficient $\beta_{k \tau}, k=1,2,3$ and 4 , is a function of the variance $\left(\sigma_{r_{\tau}}^{2}\right)$ which contains the components of information $\left(\sigma_{f_{\tau}}^{2}\right)$, order flow $\left(\sigma_{o_{\tau}}^{2}\right)$ and noise $\left(\sigma_{t_{\tau}}^{2}\right)$. Specifically,

$$
\begin{aligned}
\beta_{k \tau} & =\beta\left(\sigma_{r_{\tau}}^{2}\right) \\
& =\beta\left(\sigma_{f_{\tau}}^{2}, \sigma_{o_{\tau}}^{2}, \sigma_{z_{\tau}}^{2}\right)
\end{aligned}
$$

where

$\sigma_{o_{\tau}}^{2}=2\left|a_{0}+a_{1} \theta_{\tau-1}\right| \sigma_{u_{\tau-1}}^{2}, \sigma_{z_{\tau}}^{2}=2\left|\theta_{\tau-1}-a_{0}-a_{1} \theta_{\tau-1}\right| \sigma_{u_{\tau-1}}^{2}$ and $\sigma_{f_{\tau}}^{2}=\sigma_{r_{\tau}}^{2}+\theta_{\tau-1} \sigma_{u_{\tau-1}}^{2}$, as defined above, and $\sigma_{r_{t}}^{2}$ which equals the total variance of the returns, and $\sigma_{u_{\tau}}^{2}$ which equals the residual of the first moving average model, as shown below: ${ }^{60}$

$r_{\tau}=\theta_{\tau-1} u_{\tau-1}+u_{\tau}$

As shown in (11) in Essay 2, the informational variance is defined as the difference between the total variance and the total noise variance. If the total variance does not significantly change, then there will be a significant negative relation between the informational variance and the measure of the total noise variance. In order to remove the negative correlation between the informational and noise variances, the residual of the regression of the informational variance measure (in (10) in essay 2) on the noise variance measure (in (11) in Essay 2) is used as the informational variance measure in the equation in (3), i.e., $\hat{\sigma}_{f_{r, e}}^{2}$. The measure of the total noise variance in (3) is the absolute noise variance measure. Moreover, the absolute value of the noise variance measure represents the deviation of the current price from the efficient price. The Appendix in Essay 3 shows the decomposition of the total noise variance into the order flow variance

\footnotetext{
${ }^{60}$ See appendix 3.2 for the coefficients, $a_{0}$ and $a_{1}$.
} 
component and the remaining pure noise variance component. The total variance and the measures of informational, order flow, and noise variance are estimated before the regression model given in (4) and (5) is determined.

This study redefines $\beta_{k \tau}$ from (2) in terms of two linear functions employing the variances of information, order flow, and noise as follows:

$$
\begin{aligned}
& \beta_{k \tau}=\beta_{k}+\lambda_{r k}\left(\sigma_{r_{\tau}}^{2}-\overline{\hat{\sigma}}_{r_{\tau}}^{2}\right) \\
& \beta_{k \tau}=\beta_{k}+\lambda_{f k}\left(\hat{\sigma}_{f_{\tau, e}}^{2}-\overline{\hat{\sigma}}_{f_{\tau}}^{2}\right)+\lambda_{o k}\left(\hat{\sigma}_{o_{\tau}}^{2}-\overline{\hat{\sigma}}_{o_{\tau}}^{2}\right)+\lambda_{z k}\left(\hat{\sigma}_{z_{\tau}}^{2}-\overline{\hat{\sigma}}_{z_{\tau}}^{2}\right), \text { for } k=1,2,3 \text { and } 4
\end{aligned}
$$

The estimated informational variance $\left(\hat{\sigma}_{f_{\tau, e}}^{2}\right)$, the order flow variance $\left(\hat{\sigma}_{o_{\tau}}^{2}\right)$ and the noise variance measures $\left(\hat{\sigma}_{z_{\tau}}^{2}\right)$ are demeaned by removing their simple mean values. ${ }^{61}$ Hence, the unconditional mean of the coefficients $\left(\beta_{k \tau}\right)$ will be the same value as the constant $\left(\beta_{k}\right)$ in equation (5). Specifically,

$$
\begin{aligned}
& E\left(\beta_{k \tau}\right)=\beta_{k}+\lambda_{r k} E\left(\sigma_{r_{\tau}}^{2}-\overline{\hat{\sigma}}_{r_{\tau}}^{2}\right)=\beta_{k} \\
& E\left(\beta_{k \tau}\right)=\beta_{k}+\lambda_{f k} E\left(\hat{\sigma}_{f \tau}^{2}-\overline{\hat{\sigma}}_{f \tau}^{2}\right)+\lambda_{o k} E\left(\hat{\sigma}_{o \tau}^{2}-\overline{\hat{\sigma}}_{o \tau}^{2}\right)+\lambda_{z k} E\left(\hat{\sigma}_{z \tau}^{2}-\overline{\hat{\sigma}}_{z \tau}^{2}\right)=\beta_{k},
\end{aligned}
$$

for $k=1,2,3$ and 4

The operational specifications of the regression model in (1) for (4) and (5) are as follows:

Model 1 (The combination of (1) and (4)):

$$
\begin{aligned}
r_{\tau}=\alpha_{0}+\alpha_{1} r_{\tau-1} & +\beta_{1} \mathrm{NetVol}_{1 \tau}+\lambda_{r 1} \tilde{\sigma}_{r \tau}^{2} \mathrm{NetVol}_{1 \tau}+\beta_{2} \mathrm{NetVol}_{2 \tau}+\lambda_{r 2} \tilde{\sigma}_{r \tau}^{2} \mathrm{NetVol}_{2 \tau} \\
& +\beta_{3} \mathrm{NetVol}_{3 \tau}+\lambda_{r 3} \tilde{\sigma}_{r \tau}^{2} \mathrm{NetVol}_{3 \tau}+\beta_{4} \mathrm{NetVol}_{4 \tau}+\lambda_{r 4} \tilde{\sigma}_{r \tau}^{2} \mathrm{NetVol}_{4 \tau}+\varepsilon_{\tau}
\end{aligned}
$$

\footnotetext{
${ }^{61}$ Essay 2 shows the decomposition of the variance into the informational variance component and the total noise variance component.
} 
Model 2 (The combination of (1) and (5)):

$$
\begin{aligned}
r_{\tau}=\alpha_{0}+\alpha_{1} r_{\tau-1} & +\beta_{1} \mathrm{NetVol}_{1 \tau}+\lambda_{f 1} \tilde{\sigma}_{f \tau}^{2} \mathrm{NetVol}_{1 \tau}+\lambda_{o 1} \tilde{\sigma}_{o \tau}^{2} \mathrm{NetVol}_{1 \tau}+\lambda_{z 1} \tilde{\sigma}_{z \tau}^{2} \mathrm{NetVol}_{1 \tau} \\
& +\beta_{2} \mathrm{NetVol}_{2 \tau}+\lambda_{f 2} \tilde{\sigma}_{f \tau}^{2} \mathrm{NetVol}_{2 \tau}+\lambda_{o 2} \tilde{\sigma}_{o \tau}^{2} \mathrm{NetVol}_{2 \tau}+\lambda_{z 2} \tilde{\sigma}_{z \tau}^{2} \mathrm{NetVol}_{2 \tau} \\
& +\beta_{3} \mathrm{NetVol}_{3 \tau}+\lambda_{f 3} \tilde{\sigma}_{f \tau}^{2} \mathrm{NetVol}_{3 \tau}+\lambda_{o 3} \tilde{\sigma}_{o \tau}^{2} \mathrm{NetVol}_{3 \tau}+\lambda_{z 3} \tilde{\sigma}_{\tau \tau}^{2} \mathrm{NetVol}_{3 \tau} \\
& +\beta_{4} \mathrm{NetVol}_{4 \tau}+\lambda_{f 4} \tilde{\sigma}_{f \tau}^{2} \mathrm{NetVol}_{4 \tau}+\lambda_{o 4} \tilde{\sigma}_{o \tau}^{2} \mathrm{NetVol}_{4 \tau}+\lambda_{z 4} \tilde{\sigma}_{z \tau}^{2} \mathrm{NetVol}_{4 \tau}+\varepsilon_{\tau}
\end{aligned}
$$

where $\quad \tilde{\sigma}_{r_{\tau}}^{2} \equiv \sigma_{r_{\tau}}^{2}-\overline{\hat{\sigma}}_{r_{\tau}}^{2}, \quad \tilde{\sigma}_{f \tau}^{2} \equiv \hat{\sigma}_{f \tau}^{2}-\overline{\hat{\sigma}}_{f \tau}^{2}, \quad \tilde{\sigma}_{o \tau}^{2} \equiv \hat{\sigma}_{o \tau}^{2}-\overline{\hat{\sigma}}_{o \tau}^{2} \quad$ and $\quad \tilde{\sigma}_{z \tau}^{2} \equiv \hat{\sigma}_{z \tau}^{2}-\overline{\hat{\sigma}}_{z \tau}^{2}$. The regressions in (8) and (9) will show which type of trader generates a profitable trading pattern. Before the examination of the trading volume reaction of CTI $k$ to different types of price changes (informational, order flow, and noise price changes), the regression in (8) will show whether traders have differing trading patterns for different sizes of price changes. ${ }^{62}$ If $\lambda_{\text {rk }}$ is not zero, then CTIk possesses a different demand schedule for different price change sizes. If $\lambda_{r k}<0$, then CTIk has a more profitable trading pattern when price changes are larger. The regression for Model 2, i.e. equation (9), provides us a richer examination concerning how traders' trading patterns are different for different types of information in relation to the null hypotheses sated in $\mathrm{H}_{1}$ to $\mathrm{H}_{5}$.

As previously discussed in section 3.3.1, information-motivated traders will sell (buy) immediately after the positive (negative) information causes price to change. This creates a negative relation between trading volume and current returns. Hence, an information-motivated trader group will possess a negative coefficient for their net trading volume. If a trader group has knowledge about the processes of the time-varying information arrivals and noise, then the coefficients in (7) will be significantly negative,

\footnotetext{
${ }^{62}$ Equations (4) and (5) show the relation between $\beta_{k \tau}$ and the size of the variances. Recall that the variance is calculated as the square of the 15 minute price change, thereby providing the direct relation between price change and $\beta_{k \tau}$.
} 
since the trader group will utilize its knowledge to maximize its profits from transactions; moreover, in this case null hypothesis $1\left(\mathrm{HO}_{1}\right)$ is rejected. Because information arrivals affecting price increases (decreases) is assumed to be fully reflected into prices as soon as the information arrival occurs, traders who are aware of information will buy (sell) immediately before the price change occurs. ${ }^{63}$

Furthermore, if a trader group differentiates price changes caused by information arrivals from price changes caused by noise, then the coefficient of the noise variance measure $\left(\lambda_{z k}\right)$ will be more negative than the coefficient of the informational variance measure. This occurs because the price changes caused by the noise component represent the deviation of the price from the efficient price. The stronger negative coefficient for the noise variance measure $\left(\lambda_{z k}\right)$ reflects the reduction in the deviation of price from the efficient price. Hence, if the coefficient of the noise variance measure $\left(\lambda_{z k}\right)$ is more negative than that of the informational variance $\left(\lambda_{f k}\right)$, then null hypothesis $2\left(\mathrm{HO}_{2}\right)$ is rejected.

Based on the null hypotheses $\mathrm{HO}_{3}$ and $\mathrm{HO}_{4}$, the general public traders are assumed to be noise traders. Because the general public traders are assumed to be noise traders, coefficients $\lambda_{z 4}$ and $\lambda_{f 4}$ reflect trading volume reactions by the noise traders to both the noise and the informational factors in price changes. Hence the coefficients $\lambda_{z 4}$ and $\lambda_{f 4}$

\footnotetext{
${ }^{63}$ If information is not fully reflected into price at the moment it arrives, then the price series will be positively autocorrelated. Consequently, if informed traders know that this resultant positive autocorrelation of the price changes is due to the existence of old information, then these informed traders will cause a momentum trading pattern in prices. However, in this study, autocorrelated price changes due to old information are considered to be noisy price changes, as detailed in Essay2. The price change which is not explained by the autocorrelation is considered as the price change due to new information arrivals even though the price change is caused by old information.
} 
are compared to the corresponding coefficients of floor traders $\left(\lambda_{z k}\right.$ and $\lambda_{f k}, k=1,2$ and 3) to test null hypotheses $3\left(\mathrm{HO}_{3}\right)$ and $4\left(\mathrm{HO}_{4}\right)$. If $\lambda_{z k}<\lambda_{z 4}, k=1,2$ and 3 , then the floor traders show stronger profitable trading volume reactions to price changes caused by the autocorrelated noise price behavior than do the general public and therefore we would reject $\mathrm{H}_{3}$. If $\lambda_{f k}<\lambda_{f 4}, k=1,2$ and 3 , then the floor traders show stronger profitable trading volume reactions to price changes caused by information arrivals than do the general public and therefore we would reject $\mathrm{HO}_{4}$.

If $\lambda_{o k}>0$ then CTIk has knowledge of the direction of short-term price changes from the order flow information. If $\lambda_{o k}>\lambda_{o 4}, k=1,2$ and 3 , then the floor traders have a better knowledge of the direction of short-term price changes than the general public traders, and then we would reject $\mathrm{H}_{5}$. The following statistical hypotheses examine the noisy rational expectations models explained in section 3.1.

$\mathrm{HO}_{1}: \lambda_{f k}=\lambda_{z k}=0$, for $k=1,2,3$ and 4

$\mathrm{H}_{2}: \lambda_{f k}=\lambda_{z k}$, for $k=1,2,3$ and 4

$\mathrm{H}_{3}: \lambda_{z k}=\lambda_{z 4}$, for $k=1,2$ and 3

$\mathrm{HO}_{4}: \lambda_{f k}=\lambda_{f 4}$, for $k=1,2$ and 3

$\mathrm{H}_{5}: \lambda_{\text {ok }}=\lambda_{\text {o4 }}$, for $k=1,2$ and 3

\subsection{Data}

This study employs fifteen minute futures data from The Chicago Board of Trade (CBOT) for interest rate futures (10-year T-notes), agricultural futures (wheat and corn), 
and stock index futures (Dow Jones) for years 2003 to 2006. This data includes aggregate volume for both purchases and sales for each recorded trade price for each fifteen minutes interval in the day, with all volume distinguished by four types of traders. The trade volumes are distinguished by the following customer trade indicators (CTI):

CTI1: Volume for the local market maker's own accounts. These market makers provide short-term liquidity to the market in response to the needs of other traders. CTI2: Volume for institutional house accounts. Institutional traders initiate speculating and hedging trades. They are informed of the order flow on the floor and possess more homogeneous beliefs than do other trader groups.

CTI3: Volume for floor traders executing trades for other floor traders.

CTI4: Volume for any other type of off-the-floor traders, called the general public. The general public does not have access to the floor and therefore are not privy to order flow information. Hence these traders typically can not distinguish information signals from noise, and thus possess more heterogeneous beliefs than do floor traders.

It is well known that return variances have strong intraday seasonal components. Hence, this seasonal component could easily lead to erroneous statistical inferences for microstructure studies if it is not removed from the intraday data. This study adjusts for the seasonal components inherent in the intraday data. The procedure to filter the returns series is detailed in Essay 1. The non-seasonal intraday returns possess both the interday and the intraday variance components. The interday variance components are removed from the return variance to examine only the short-run information components in price changes. Furthermore, the informational variance, the order flow information variance, and the noise variance components are decomposed from the non-seasonal intraday 
return variance, which are adjusted for the interday variance components. The resultant return series is used in the regression model given in (8) and (9) as the dependent variable and the lagged dependent regressor.

This study employs the squared returns as the measure of the total conditional variance of the returns $\left(\sigma_{r_{\tau}}^{2} \equiv r_{\tau}^{2}\right.$ ), rather than using the conditional variances obtainable from a GARCH process. The reason for using the squared returns as the measure of the total variance arises from the fifteen minute frequency interval employed in this study. The use of high frequency returns, especially since they are adjusted for the interday variance component, reflect only the information from short-term price changes. Hence, the use of an ARMA/GARCH process would smoothes out the short-term price changes, resulting in a significant loss of information when examining the high frequency dataset. In a similar way the innovations in (6) in Essay 2 were squared to determine the measure of the conditional variance of the moving average innovations $\left(\sigma_{u_{\tau}}^{2} \equiv u_{\tau}^{2}\right)$. From the squared innovations, the order flow variance measure and the non-order flow noise variance measure in (2) are computed.

\subsection{Results}

Table 3.1 provides an initial examination of the trading patterns of each type of CTI trader via the preliminary correlation coefficients between the non-seasonal intraday returns and the net trading volumes. The negative correlation coefficients for the floor traders (CTI1, 2, and 3) show that they buy (sell) if prices rise (fall). On the other hand, a positive correlation coefficient for the general public traders (CTI4) show that they buy 
(sell) if prices fall (rise). The results of the trading patterns in relation to the informational and noisy variance components analyzed by the multivariate regression analysis are presented next.

The relation between information, volatility, and trading volumes are analyzed in Table 3.2 by employing the regression models given in (8) and (9). ${ }^{64}$ The negative coefficients of the net trading volume for the current period and the positive coefficients for the previous period, as given in Panels A and B of Table 3.2, show that the floor traders have profitable information-motivated demand functions, whereas the reverse is true for the general public. In particular, the positive coefficients of the net trading volume for the previous period show that floor traders buy (sell) immediately before price increases (decreases). Significant negative coefficients for the total variance (TotalVar) in Panel A show that floor traders profitable trading patterns becomes stronger when the variance of price change becomes larger, whereas the general public traders do not show any different trading patterns for different sizes of price changes. Panel B of Table 3.2 estimates the coefficients from the decomposition of the total variance from Panel A into its three different components, namely informational variance (InforVar), order flow variance (OrderFlowVar) and noise variance (AbsNoiseVar).

In Panel B of Table 3.2 the significance of the coefficients of the informational variance and the noise variance measures test null hypothesis $1\left(\mathrm{HO}_{1}\right)$. The significantly negative coefficients for the informational variance components (InforVar, $\lambda_{f k}$ ) for CTI2

\footnotetext{
${ }^{64}$ The regression in (4) shows the trading patterns of each type of traders in response to different types of price changes, where the price changes are caused by ether information arrivals or noise. The regression analysis in (4) does not directly examine the relation between volatility and trading volumes. Daigler and Wiley, (1999), Chang, Chou, and Nelling (2000) and Wang (2002) examine the direct volatility-volume relation.
} 
in panel A of Table 3.2 show that the market makers (CTI1) and institutional traders (CTI2) sell (buy) more contracts when the contemporaneous return increases (decreases) are larger and are due to information variance. ${ }^{65}$ The other floor traders (CTI3) also sell (buy) more contracts if the current price increases (decreases) are larger, with the exception of Dow Jones futures contracts. ${ }^{66}$ These findings concerning the trading patterns of differently motivated traders (their information-motivated demand/supply schedule) in relation to the changes in the informational variance reject $\mathrm{H}_{1}$. In addition, these results are consistent with the trading volume reactions of floor traders to price changes being correlated with the time-varying characteristics of information arrivals.

Informed traders' negative relation between the contemporaneous return and demand will be stronger if price changes are caused by noise factors rather than by information arrivals, since informed traders are able to differentiate noise from new information arrivals. This stronger negative relation between the contemporaneous relation and demand should be reflected in the regression results by larger negative coefficients for the noise variance measure relative to the informational variance measure (AbsNoiseVar $\left(\lambda_{z k}\right)<\operatorname{InforVar}\left(\lambda_{f k}\right)<0$ ). Table 3.2 shows that the net trading volume of the floor traders has larger negative coefficients for the noise variance measures relative to the informational variance, especially for CTI2 traders. For example, the coefficients of CTI2 net volume are -1.985 (InforVar) and -10.394 (AbsNoiseVar) for the

\footnotetext{
${ }^{65}$ The negative relation between contemporaneous return and the demand (trading) schedule is equivalent to the positive relation between the expected returns and the demand schedule. Hence, buying (selling) when the contemporaneous return decreases (increases) is equivalent to selling (buying) when the expected return decreases (increases).

${ }^{66}$ Also, the other floor traders (CTI3) do not show profitable trading patterns for the Dow Jones contract. Based on the results in Panel A and B of Table 3.2, they sell before prices increase and buy when expected prices decrease.
} 
10-year T-note futures. On the other hand, the net trading volume of the general public traders for the Dow Jones contract does not possess larger negative coefficients for the noise variance measures, suggesting the general public traders are not informed traders in that they do not distinguish noise from information.

Floor traders possess order flow information in conjunction with contemporaneous and past return behavior. Therefore, although there is a negative relation between the contemporaneous return and the demand of informed traders, as detailed in Appendix 3.1, floor traders' demand will be positively related to a determinant of the contemporaneous return if that determinant possesses a forecast of the expected return. Appendix 3.2 discusses how to decompose the total noise variance into the order flow component and the pure noise component. The coefficient for the order flow component (OrderFlowVar, $\lambda_{o k}$ ) in Panel B of Table 3.2 supports the idea that the coefficients $\lambda_{o k}$ for the floor traders are significantly positive; this is especially true for CTI2, whereas the order flow coefficient for the CTI4 general public traders coefficient values are insignificant. ${ }^{67}$

Panel A of Table 3.3 provides the statistical significance results to test whether the coefficients of the noise variance measures are statistically less than the coefficients of the informational variance. These valufes are determined by comparing the

\footnotetext{
${ }^{67}$ See Daigler and Wiley (1999), Massimb and Phelps (1994), and Kurov and Lasser (2004) regarding order flow information and floor traders. Daigler and Wiley (1999) find that the positive relation between volatility and volume is driven by the general public, who are not on the floor and therefore they cannot distinguish what price changes are due to information arrivals and what price changes are due to noise from liquidity demand. Massimb and Phelps (1994) detail floor traders' strategic advantages in the pits, such as recognizing the subsequent direction of the market and the ability to execute certain trading strategies. Moreover, floor traders have other advantages, especially when both floor and electronic markets exist in parallel. For example, as Kurov and Lasser (2004) show, when large stock index futures orders are sent to the floor for execution, then market makers first make trades for their own account in the electronic markets before executing the large customer trades on the floor.
} 
information coefficients to the absolute noise coefficients in Table 3.2. These results show that the noise component possesses a much larger negative coefficient than the information component for the CTI2 institutional traders. This supports a stronger negative relation between the contemporaneous return and the trading demand for the noise component relative to the information component. The results from Table 3.3 also show that a stronger negative relation between the contemporaneous return and demand exists for current price changes caused by the noise component (i.e. NoiseVar $<$ InforVar $<0$ ) for 10-Year T-notes and Dow Jones for the CTI1 traders and for 10-Year T-notes, wheat and corn for the CTI3 traders. On the other hand, the results from Panel A of Table 3.3 show that the net trading volume of the general public traders do not possess significantly larger negative noise variance coefficients relative to the informational variance coefficients. Hence, trading volume reactions to price changes caused by information are significantly different from those caused by noise for floor traders, whereas those of the general public traders are not. As a result, $\mathrm{HO}_{2}$ is rejected.

As mentioned in the methodology section, if the prediction by the noisy rational expectations model is valid, then informed traders exhibit stronger negative net volume reactions to contemporaneous price changes than do the general public whether the price changes are caused by noise or information (see $\mathrm{HO}_{3}$ and $\mathrm{HO}_{4}$ ). The test results in Panel $\mathrm{B}$ and $\mathrm{C}$ show that the net volume reactions of floor traders to price changes caused by either noise or information are statistically less than those of the general public, with the exception of the Dow Jones contract for the other (CTI3) floor traders.

Previously, I showed in Panel B of Table 3.2 that floor traders exhibit knowledge of order flow information, since the OrderFlowVar coefficients $\left(\lambda_{o k}\right)$ were significantly 
positive whereas the general public traders coefficients were not. The p-values in Panel D of Table 3.3 reaffirm this previous finding concerning the order flow information in Panel B of Table 3.2 in that the net volume reactions of floor traders to the order flow component of the contemporaneous price changes are statistically larger than those of the general public, with the exception of the Dow Jones contract for other floor traders (CTI3).

In conclusion, the floor traders' current demand schedule exhibits a positive relation to expected return and a negative relation to contemporaneous return. Their information-motivated trading pattern is stronger for contemporaneous price changes caused by noise than for price changes caused by information arrivals. On the other hand, the trading pattern of the general public traders is positively correlated to contemporaneous price changes.

\subsection{Conclusions}

This study applies the seasonality filtering process developed in Essay 1 to examine the noisy rational expectations model. In particular, I analyze the relation between information, order flow, noise and traders' demand functions for 10-year Tnotes, Dow Jones, wheat and corn futures. The noisy rational expectations model states that informed traders' expected returns have a lower correlation with current noise than do uninformed general public traders. In addition, informed traders' current demand is negatively related to an increase in contemporaneous noise and information arrivals. The reason for the negative relation between trading demand and both information and noise 
is that informed traders will sell an asset if the current return increases, whether the increase in the current return is caused by noise or by information. Furthermore, informed traders' current demand is more negatively related to the increase in the current returns caused by noise than by information because informed traders can distinguish noise from information. As Massimb and Phelps (1994) point out, floor traders who observe order flow from the trading floor have an advantage in determining how prices will change based on the contemporaneous and past returns. Therefore, although there is a negative relation between the contemporaneous return and the trading demand of informed traders, floor traders' demand will be positively related to a determinant of the contemporaneous return if that determinant is a factor affecting expected return.

In order to obtain the appropriate price series for analysis, the seasonal variance and the interday variance components are removed before the analysis of the relation between information, order flow, noise, and trading activity is undertaken. The procedure to filter out the intraday seasonal variance component is detailed in Essay 1. The interday variance component also is removed from the return variance in order to concentrate on the short-run information component in price changes. In addition, the non-seasonal intraday return variance is decomposed into the informational variance, order flow variance, and the noise variance components. The procedure to decompose the variance into the informational, order flow and noise components is detailed in Essay 2 and Appendix 3.2 in this study (Essay 3). Subsequently, the decomposed informational, order flow and noise variance components are used to examine the trading demand function in response to time-varying information, order flow and noise. 
The results of the analysis of the data are consistent with prediction from the noisy rational expectations model in that the floor traders' demand function possesses the properties of an information-motivated demand schedule. This relation is supported by the floor traders' current demand schedule exhibiting a positive relation to the expected return and a negative relation to the contemporaneous return. This information-motivated trading pattern is stronger for contemporaneous price changes caused by noise than for price changes caused by information arrivals. Alternatively, the trading pattern of general public traders is positively correlated to contemporaneous price changes, showing they do not possess an information-motivated demand function. Finally, the results show that floor traders have order flow information whereas the general public does not. 


\section{Table 3.1: Correlations Coefficients between the Non-seasonal Intraday Returns and Net Trading Volumes by CTI}

Table 3.1 shows the Pearson correlation coefficients between the non-seasonal intraday returns and net trading volumes by type of trader (CTI). NetVol1, NetVol2, NetVol3 and NetVol4 represent the buy volume less the sell volume for the CTI1, CTI2, CTI3 and CTI4 traders, respectively. Statistical significance at less than the $5 \%$ level is shown in bold.

Panel A: Correlation coefficients of 10-Year T-notes

\begin{tabular}{ccccc}
\hline & NetVol1 & NetVol2 & NetVol3 & NetVol4 \\
\hline Returns & $\mathbf{- 0 . 1 2 0}$ & $\mathbf{- 0 . 0 5 6}$ & $\mathbf{- 0 . 0 8 1}$ & $\mathbf{0 . 1 5 0}$ \\
NetVol1 & & $\mathbf{- 0 . 1 9 4}$ & $\mathbf{0 . 2 2 8}$ & $\mathbf{- 0 . 7 6 9}$ \\
NetVol2 & & & $-\mathbf{0 . 1 7 7}$ & $\mathbf{- 0 . 0 6 1}$ \\
NetVol3 & & & & $\mathbf{- 0 . 5 1 4}$ \\
\hline
\end{tabular}

Panel B: Correlation coefficients of Dow Jones

\begin{tabular}{ccccc}
\hline & NetVol1 & NetVol2 & NetVol3 & NetVol4 \\
\hline Returns & $\mathbf{- 0 . 0 9 9}$ & $\mathbf{- 0 . 1 0 0}$ & 0.003 & $\mathbf{0 . 0 8 8}$ \\
NetVol1 & & $\mathbf{0 . 1 5 1}$ & $\mathbf{0 . 1 1 7}$ & $\mathbf{- 0 . 7 2 8}$ \\
NetVol2 & & & $\mathbf{0 . 0 2 5}$ & $\mathbf{- 0 . 2 9 4}$ \\
NetVol3 & & & & $\mathbf{- 0 . 2 8 5}$ \\
\hline
\end{tabular}

Panel C: Correlation coefficients between Wheat

\begin{tabular}{ccccc}
\hline & NetVol1 & NetVol2 & NetVol3 & NetVol4 \\
\hline Returns & $\mathbf{- 0 . 1 5 1}$ & $\mathbf{- 0 . 0 9 4}$ & $\mathbf{- 0 . 1 1 9}$ & $\mathbf{0 . 1 7 3}$ \\
NetVol1 & & $\mathbf{- 0 . 0 5 0}$ & $\mathbf{0 . 2 1 8}$ & $\mathbf{- 0 . 6 6 0}$ \\
NetVol2 & & & $\mathbf{0 . 0 5 5}$ & $\mathbf{- 0 . 1 7 9}$ \\
NetVol3 & & & & $\mathbf{- 0 . 5 2 1}$ \\
\hline
\end{tabular}

Panel D: Correlation coefficients between Corn

\begin{tabular}{ccccc}
\hline & NetVol1 & NetVol2 & NetVol3 & NetVol4 \\
\hline Returns & $\mathbf{- 0 . 0 2 7}$ & $\mathbf{- 0 . 1 2 2}$ & $\mathbf{- 0 . 1 6 9}$ & $\mathbf{0 . 1 1 4}$ \\
NetVol1 & & $\mathbf{- 0 . 1 5 2}$ & -0.013 & $\mathbf{- 0 . 7 0 6}$ \\
NetVol2 & & & -0.003 & $\mathbf{- 0 . 1 5 1}$ \\
NetVol3 & & & & $\mathbf{- 0 . 2 8 1}$ \\
\hline
\end{tabular}




\section{Table 3.2: Regression Results of the Noisy Rational Expectations Models}

Table 3.2 shows the regression results of the non-seasonal intraday returns against the net trading volumes. NetVol1, NetVol2, NetVol3 and NetVol4 represent the net trading volumes (buy volume less sell volume) for the CTI1, CTI2, CTI3 and CTI4 traders, respectively. TotalVar, InforVar, OrderFlowVar and NoiseVar represent $\lambda_{k}, \lambda_{f k}, \lambda_{o k}$ and $\lambda_{z k}$, respectively. The net volume coefficients are multiplied by a factor of 10,000. Statistical significance at less than the $5 \%$ level is shown in bold. The symbol $\dagger$ represents the statistical significance at a $10 \%$ level. The regression models are as follows:

$$
\begin{aligned}
& r_{\tau}=\alpha_{0}+\alpha_{1} r_{\tau-1}+\beta_{1 \tau} N_{\text {etVol }}+\beta_{2 \tau} \operatorname{NetVol}_{2 \tau}+\beta_{3 \tau} \operatorname{NetVol}_{3 \tau}+\beta_{4 \tau} \operatorname{NetVol}_{4 \tau}+\varepsilon_{\tau} \\
& \beta_{k \tau}=\beta_{k}+\lambda_{r k}\left(\sigma_{r_{\tau}}^{2}-\overline{\hat{\sigma}}_{r_{\tau}}^{2}\right) \text { for } k=1,2,3 \text { and } 4 \text { and Panel A. } \\
& \beta_{k \tau}=\beta_{k}+\lambda_{f k}\left(\hat{\sigma}_{f_{\tau}}^{2}-\overline{\hat{\sigma}}_{f_{\tau}}^{2}\right)+\lambda_{o k}\left(\hat{\sigma}_{o_{\tau}}^{2}-\overline{\hat{\sigma}}_{o_{\tau}}^{2}\right)+\lambda_{z k}\left(\hat{\sigma}_{z_{\tau}}^{2}-\overline{\hat{\sigma}}_{z_{\tau}}^{2}\right) \text { for } k=1,2,3 \text { and } 4 \text { and Panel B. }
\end{aligned}
$$

Panel A

\begin{tabular}{lrrrr}
\hline & $\begin{array}{r}10 \text {-year } \\
\text { T-notes }\end{array}$ & Dow Jones & Wheat & Corn \\
\cline { 2 - 5 } Intercept & 0.006 & 0.002 & -0.004 & $\mathbf{- 0 . 0 1 1}$ \\
Ret $(\tau-1)$ & $\mathbf{0 . 7 5 8}$ & $\mathbf{0 . 7 7 6}$ & $\mathbf{0 . 7 6 2}$ & $\mathbf{0 . 7 7 4}$ \\
& & & & \\
NetVol1 & $\mathbf{- 0 . 7 4 2}$ & $\mathbf{- 8 . 7 1 0}$ & $\mathbf{- 0 . 8 3 8}$ & -0.219 \\
TotalVar & $\mathbf{- 0 . 4 1 1}$ & $-0.825 \dagger$ & $\mathbf{- 1 . 0 7 2}$ & $\mathbf{- 0 . 2 4 7}$ \\
NetVol1 $(\tau-1)$ & $\mathbf{0 . 4 3 8}$ & 3.533 & 0.220 & $\mathbf{- 0 . 8 1 5}$ \\
& & & & \\
NetVol2 & 0.364 & $\mathbf{- 8 . 3 4 0}$ & -0.671 & $\mathbf{- 1 . 5 3 0}$ \\
TotalVar & $\mathbf{- 1 . 5 5 7}$ & $\mathbf{- 5 . 4 7 2}$ & $\mathbf{- 1 . 7 8 1}$ & $\mathbf{- 1 . 0 1 3}$ \\
NetVol2 $(\tau-1)$ & $\mathbf{0 . 6 9 2}$ & $\mathbf{1 0 . 2 0 9}$ & $\mathbf{4 . 1 7 6}$ & $\mathbf{1 . 2 8 7}$ \\
& & & & \\
NetVol3 & -0.363 & $\mathbf{5 . 4 8 4}$ & -0.433 & $\mathbf{- 4 . 0 5 2}$ \\
TotalVar & $\mathbf{- 0 . 5 6 0}$ & -0.221 & $\mathbf{- 1 . 4 2 1}$ & $\mathbf{- 1 . 5 3 4}$ \\
NetVol3 $(\tau-1)$ & 0.200 & -2.314 & $\mathbf{2 . 1 5 2}$ & $\mathbf{0 . 7 4 9}$ \\
NetVol4 & $0.335 \dagger$ & 0.511 & 0.268 & $\mathbf{- 0 . 4 0 3}$ \\
TotalVar & 0.058 & 0.362 & 0.133 & 0.122 \\
NetVol4 $(\tau-1)$ & $\mathbf{- 0 . 5 8 2}$ & $-\mathbf{2 . 2 1 7}$ & -0.432 & $\mathbf{- 1 . 0 3 4}$ \\
R-Square & 0.598 & 0.614 & 0.614 & 0.643 \\
N & 25,379 & 30,793 & 12,187 & 12,184 \\
\hline
\end{tabular}


Table 3.2: Regression Results of the Noisy Rational Expectations Models (Continued)

Panel B

\begin{tabular}{|c|c|c|c|c|}
\hline & $\begin{array}{l}\text { 10-year } \\
\text { T-notes }\end{array}$ & Dow Jones & Wheat & Corn \\
\hline Intercept & 0.005 & 0.002 & -0.004 & -0.011 \\
\hline Ret (t-1) & 0.759 & 0.776 & 0.764 & 0.776 \\
\hline NetVol1 & -0.761 & -8.900 & -0.814 & -0.180 \\
\hline InforVar & -0.586 & -1.980 & -1.432 & $-0.164 \dagger$ \\
\hline AbsNoiseVar & -4.670 & -16.205 & -4.763 & -0.730 \\
\hline OrderFlowVar & 1.306 & 4.837 & 1.820 & 0.137 \\
\hline NetVol1 $(\tau-1)$ & 0.447 & 3.451 & 0.239 & -0.852 \\
\hline NetVol2 & 0.251 & -8.633 & -0.876 & -1.459 \\
\hline InforVar & -1.985 & -6.459 & -2.422 & -1.374 \\
\hline AbsNoiseVar & -10.394 & -33.217 & -13.422 & -6.319 \\
\hline OrderFlowVar & 3.106 & 8.266 & 3.826 & 2.639 \\
\hline $\operatorname{NetVol2}(\tau-1)$ & 0.625 & 9.997 & 4.122 & 1.162 \\
\hline NetVol3 & -0.307 & 5.716 & -0.375 & -3.989 \\
\hline InforVar & -0.881 & 0.934 & -3.094 & -2.300 \\
\hline AbsNoiseVar & -7.137 & -5.809 & -25.972 & -9.254 \\
\hline OrderFlowVar & 2.031 & -1.439 & 6.261 & 4.187 \\
\hline $\operatorname{NetVol3}(\tau-1)$ & 0.187 & -2.324 & 2.299 & $0.682 \dagger$ \\
\hline NetVol4 & $0.367 \dagger$ & 0.701 & $0.560 \dagger$ & -0.370 \\
\hline InforVar & 0.049 & 0.571 & -0.044 & 0.220 \\
\hline AbsNoiseVar & -1.179 & -3.094 & -0.414 & 0.812 \\
\hline OrderFlowVar & 0.307 & 0.341 & -0.012 & -0.366 \\
\hline NetVol4 $(\tau-1)$ & -0.570 & -2.284 & -0.349 & -1.065 \\
\hline R-Square & 0.600 & 0.614 & 0.614 & 0.645 \\
\hline $\mathrm{N}$ & 25,379 & 30,793 & 12,187 & 12,184 \\
\hline
\end{tabular}




\section{Table 3.3: Test Results for the Noisy Rational Expectations Model}

Table 3.3 shows the test results as specified in sections 3.1 and 3.2. The tests are based on the regression coefficients of models given in (1) and (2). The regression model is as follows:

$$
\begin{aligned}
& r_{\tau}=\alpha_{0}+\alpha_{1} r_{\tau-1}+\beta_{1 \tau} \operatorname{NetVol}_{1 \tau}+\beta_{2 \tau} \operatorname{NetVol}_{2 \tau}+\beta_{3 \tau} \operatorname{NetVol}_{3 \tau}+\beta_{4 \tau} \operatorname{NetVol}_{4 \tau}+\varepsilon_{\tau} \\
& \beta_{k \tau}=\beta_{k}+\lambda_{f k}\left(\hat{\sigma}_{f_{\tau}}^{2}-\overline{\hat{\sigma}}_{f_{\tau}}^{2}\right)+\lambda_{o k}\left(\hat{\sigma}_{o_{\tau}}^{2}-\overline{\hat{\sigma}}_{o_{\tau}}^{2}\right)+\lambda_{z k}\left(\hat{\sigma}_{z_{\tau}}^{2}-\overline{\hat{\sigma}}_{z_{\tau}}^{2}\right), k=1,2,3 \text { and } 4,
\end{aligned}
$$

The null hypotheses are:

$$
\begin{aligned}
& \mathrm{H}_{1}: \lambda_{f k}=\lambda_{z k}=0, \text { for } k=1,2,3 \text { and } 4 \\
& \mathrm{H}_{2}: \lambda_{f k}=\lambda_{z k}, \text { for } k=1,2,3 \text { and } 4 \\
& \mathrm{H}_{3}: \lambda_{z k}=\lambda_{z 4}, \text { for } k=1,2 \text { and } 3 \\
& \mathrm{H}_{4}: \lambda_{f k}=\lambda_{f 4} \text {, for } k=1,2 \text { and } 3 \\
& \mathrm{H}_{5}: \lambda_{o k}=\lambda_{o 4}, \text { for } k=1,2 \text { and } 3
\end{aligned}
$$

P-statistics of the F-test results are reported for the null hypotheses of $\mathrm{HO}_{2}, \mathrm{HO}_{3}, \mathrm{HO}_{4}$ and $\mathrm{HO}_{5}$. The test results of $\mathrm{HO}_{1}$ are shown by the significance of InforVar and NoiseVar in Table 3.1. If the coefficients of variance measures $\left(\lambda_{f k}\right.$ and $\lambda_{z k}$ ) in Table 3.2 (Panel B) are not negative for floor traders, then the $\mathrm{HO}_{3}$ and $\mathrm{HO}_{4}$ hypotheses are not tested. In that case, the p-statistics are marked as NA. Similarly, if the coefficients of the order flow variance measure $\left(\lambda_{o k}\right)$ in Table 3.2 (Panel B) are not positive for floor traders, then the $\mathrm{HO}_{3}$ hypothesis is not tested. In that case, the p-statistics are marked as NA.

\begin{tabular}{lcccc}
\hline \multicolumn{5}{c}{ Panel A: Test results for Hypothesis $2\left(\mathrm{HO}_{2}\right)$} \\
\hline & 10-Year & Dow & Wheat & Corn \\
T-notes & Jones & & \\
\cline { 2 - 5 } CTI1 & 0.000 & 0.005 & 0.110 & 0.339 \\
CTI2 & 0.000 & 0.021 & 0.009 & 0.000 \\
CTI3 & 0.000 & 0.568 & 0.000 & 0.000 \\
CTI4 & 0.091 & 0.370 & 0.810 & 0.255 \\
\hline
\end{tabular}

\begin{tabular}{rcccc}
\hline \multicolumn{5}{c}{ Panel B: Test results for Hypothesis $3\left(\mathrm{H}_{3}\right)$} \\
\hline & $\begin{array}{l}\text { 10-Year } \\
\text { T-notes }\end{array}$ & $\begin{array}{c}\text { Dow } \\
\text { Jones }\end{array}$ & Wheat & Corn \\
\cline { 2 - 5 } & CTI4 & CTI4 & CTI4 & CTI4 \\
\cline { 2 - 5 } CTI1 & 0.000 & 0.000 & 0.011 & 0.001 \\
CTI2 & 0.000 & 0.012 & 0.002 & 0.000 \\
CTI3 & 0.000 & 0.824 & 0.000 & 0.000 \\
\hline
\end{tabular}

Panel C: Test results for Hypothesis $4\left(\mathrm{HO}_{4}\right)$

Panel D: Test results for Hypothesis $5\left(\mathrm{H}_{5}\right)$

\begin{tabular}{cccc}
$\begin{array}{c}10-Y e a r \\
\text { T-notes }\end{array}$ & $\begin{array}{c}\text { Dow } \\
\text { Jones }\end{array}$ & Wheat & Corn \\
\hline CTI4 & CTI4 & CTI4 & CTI4 \\
\hline 0.000 & 0.000 & 0.000 & 0.000 \\
0.000 & 0.000 & 0.000 & 0.000 \\
0.000 & NA & 0.000 & 0.000
\end{tabular}

\begin{tabular}{lcccc} 
& $\begin{array}{c}\text { 10-Year } \\
\text { T-notes }\end{array}$ & $\begin{array}{c}\text { Dow } \\
\text { Jones }\end{array}$ & Wheat & Corn \\
\cline { 2 - 5 } CTI1 & 0.000 & 0.000 & 0.000 & 0.011 \\
CTI2 & 0.000 & 0.003 & 0.000 & 0.000 \\
CTI3 & 0.000 & NA & 0.000 & 0.000 \\
\hline
\end{tabular}




\section{REFERENCES}

Admati, Anat R., 1985, A Noisy Rational Expectations Equilibrium for Multi-Asset Securities Markets, Econometrica 53, 629 - 658.

Daigler, Robert T., 1997, Intraday Futures Volatility and Theories of Market Behavior, Journal of Futures Markets 17, 45 - 74.

Enders, Walter, 2004, Applied Econometric Time Series, $2^{\text {nd }}$ Ed.

Fuller, Wayne A., 1995, Introduction to Statistical Time Series, $2^{\text {nd }}$ Ed.

Grossman, Sanford, 1976, On the Efficiency of Competitive Stock Markets where Trades have Diverse Information, Journal of Finance 18, 81 - 101.

Harvey, Andrew C., 1989, Forecasting, Structural Time Series Models and the Kalman Filter

Hasbrouck, Joel, 1991a, Measuring the Information Content of Stock Trades, Journal of Finance 46, 179 - 207.

Hasbrouck, Joel, 1991b, The Summary Informativeness of Stock Trades: An Econometric Analysis, Review of Financial Studies 4, 571 - 595.

Hasbrouck, Joel, 2002, Stalking the "efficient price" in market microstructure specifications: an overview, Journal of Financial Markets 5, 329 - 339.

Kim, Oliver, and Robert E. Verrecchia, 1991, Trading Volume and Price Reactions to Public Announcements, Journal of Accounting Research 29, 302 - 321

Kurov, A., Lasser, D. J., 2004. Price dynamics in the regular and e-mini futures markets, Journal of Financial and Quantitative Analysis 39, 365 - 384.

Shalen, Chatherine, T., 1993, Volume, Volatility, and the Dispersion of Beliefs, Review of Financial Studies 6, 405 - 434.

Sharpe, William F., 1964, Capital Asset Prices: A Theory of Market Equilibrium under Conditions of Risk, Journal of Finance 19, 425 - 442.

Lintner, John, The Valuation of risk Assets and the Selection of Risky Investment in Stock Portfolios and Capital Budgets, Review of Economics and Statistics 47, 13 - 67.

Massimb, M. N., Phelps, B. D., 1994. Electronic trading, market structure and liquidity, Financial Analyst Journal 50, 39 - 50. 
Mossin, Jan, 1966, Equilibrium in a Capital Asset Market, Econometrica 34, 768 - 783.

Wang, Changyun, 2002, The Effect of Net Positions by Type of Trader on Volatility in Foreign Currency Futures Markets, Journal of Futures Markets 22, 427 - 450. 


\section{Appendix 3.1: Derivations of the random-walk plus noise model and the information-motivated traders' demand function}

This appendix derives the random-walk plus noise model and the informationmotivated traders' demand function on the basis of the noisy rational expectations model.

The model:

a. A two-period pure exchange economy. Traders have initial endowments (initial holdings) of their assets. There is no additional issuance of assets.

Assets: a riskless bond and a risky asset (stock).

b. The price of the bond is normalized to unity. There are no changes in the demand of bond because there are no changes in the price of the bond. Hence, the gain from the bond is only based on the risk-free rate. This study assumes the risk-free rate to be 0 with a loss of generality in order to obtain simplicity.

c. The total quantity of the risky asset is fixed, whereas the quality is time-varying in that the net present value (NPV) of the risky asset changes. As a result, the changes of the NPV create information, which is a factor for the demand of the risky asset.

Agents: There are $\mathrm{N}$ speculators and $\mathrm{N}$ noisy traders.

d. Noisy traders are liquidity-motivated traders. Noisy traders' demand for the risky asset is exogenously determined because their demand is random.

e. Speculators are the information-motivated traders. Speculators are rational and risk-averse. Their demand for the risky asset is determined in order to maximize the expected utility conditional on their public and individual information.

Utility function:

f. The speculators' utility is a real-valued function of wealth and represented by the HARA class negative exponential function with a risk tolerance $a_{i} \cdot{ }^{68}$ Specifically,

$U_{i}\left(W_{t, i}\right)=-\exp \left(-\frac{W_{t, i}}{a_{i}}\right)$.

The demand function:

Demand functions are derived based on the assumptions of (a) to (f) above. Wealth is a combination of the bond and the risky asset. Specifically,

$W_{t, i}=B_{i}+P_{t} X_{t, i}$

where $B_{i}$ is the dollar value of bond, and $X_{t, i}$ is the holding of the risky asset of speculator $i$. At the next period, the trader's wealth is as follows.

\footnotetext{
${ }^{68}$ The smaller is $a_{i}$, the more risk averse is the trader.
} 
$\tilde{W}_{t+1, i}=B_{i}+\tilde{P}_{t+1} X_{t, i}$

By substituting $B_{i}$ in (A2) into (A3), the equation (A3) is rewritten as follows.

$\tilde{W}_{t+1, i}=W_{t, i}+\left(\tilde{P}_{t+1}-P_{t}\right) X_{t, i}$,

The endowment for the risky asset for speculator $i$ is $X_{0, i}$ which is a constant value and assumed to be zero with the loss of generality. Hence, $X_{t, i}$ is the net demand, which is the trading volume for speculator $i$ at $P_{t}$. Assume that the wealth $\tilde{W}_{t+1, i}$ is normally distributed. Then, the expected utility is as follows. ${ }^{69}$

$E_{t, i}\left[u\left(W_{t+1, i}\right)\right]=-\exp \left(-\frac{E_{t, i}\left[W_{t+1, i}\right]}{a_{i}}+\frac{\operatorname{Var}_{t, i}\left[W_{t+1, i}\right]}{2 a_{i}^{2}}\right)$.

The representative trader's optimization problem is maximizing the expected utility specified as follows.

$\operatorname{MAX}_{X_{t, i}} J_{t, i}=E_{t, i}\left[\tilde{W}_{t+1, i}\right]-\frac{\operatorname{Var}_{t, i}\left[\tilde{W}_{t+1, i}\right]}{2 a_{i}}$,

where

$E_{t, i}\left[\tilde{W}_{t+1 i}\right]=W_{t, i}+\left(E_{t, i}\left[\tilde{P}_{t+1}\right]-P_{t}\right) X_{t, i}$,

$\operatorname{Var}_{t, i}\left[\tilde{W}_{t+1, i}\right]=X_{t, i}^{2} \operatorname{Var}_{t, i}\left[\tilde{P}_{t+1}\right]$.

The expectation on the price of the next period is made based on the speculator's information $I_{t, i}$ which include all public and individual information. The information set $I_{t, i}$ is suppressed from the expectation $E_{t, i}\left[\cdot \mid I_{t, i}\right]$ and the variance operators $\operatorname{Var}_{t, i}\left[\cdot \mid I_{t, i}\right]$.

The first order conditions of the maximization problem give us the following speculator $i$ 's demand function:

$X_{t, i}=\frac{a_{i}\left(E_{t, i}\left[\tilde{P}_{t+1}\right]-P_{t}\right)}{\operatorname{Var}_{t, i}\left[\tilde{P}_{t+1}\right]}$

or

$X_{t, i}=\rho_{t, i}\left(E_{t, i}\left[\tilde{P}_{t+1}\right]-P_{t}\right)=\rho_{t, i} E_{t, i}\left[\tilde{r}_{t+1}\right]$,

where

$\rho_{t, i} \equiv \frac{a_{i}}{\operatorname{Var}_{t, i}\left[\tilde{P}_{t+1}\right]}$, and

${ }^{69}$ If any variable $\tilde{y} \sim N(m, v)$, then $E[\exp (\tilde{y})]=\exp (m+v / 2)$. 
$E_{t, i}\left[\tilde{r}_{t+1}\right] \equiv E_{t, i}\left[\tilde{P}_{t+1}\right]-P_{t}$.

The market clearing condition when there are no noisy-traders' demand is:

$\sum_{i=1}^{N} X_{t, i}=\sum_{i=1}^{N} \rho_{t, i}\left(E_{t, i}\left[\tilde{P}_{t+1}\right]-P_{t}\right)=\sum_{i=1}^{N} X_{0, i}=0$.

By solving for $P_{t}$, the equilibrium price is obtained as follows:

$P_{t}=\frac{\sum_{i=1}^{N} \rho_{t, i} E_{t, i}\left[\tilde{P}_{t+1}\right]}{\sum_{i=1}^{N} \rho_{t, i}} \equiv E_{t}\left[\tilde{P}_{t+1}\right]$

The above equilibrium price fully gathers the diverse individual information $I_{t, i}$ about the price of the next period. Hence, the current price fully reveals all public and individual information about the price of the next period. The market clearing condition when there are noisy-traders' demand is:

$\sum_{i=1}^{N} X_{t, i}=\sum_{i=1}^{N} \rho_{t, i}\left(E_{t, i}\left[\tilde{P}_{t+1}\right]-P_{t}\right)+N \cdot Z_{t}=0$,

where $Z_{t}$ is the per-capita net liquidity demand which is not matched by liquidity supply. By solving for $P_{t}$, the equilibrium price is obtained as follows:

$P_{t}=\frac{\sum_{i=1}^{N} \rho_{t, i} E_{t, i}\left[\tilde{P}_{t+1}\right]}{\sum_{i=1}^{N} \rho_{t, i}}+\frac{N \cdot Z_{t}}{\sum_{i=1}^{N} \rho_{t, i}} \equiv E_{t}\left[\tilde{P}_{t+1}\right]+Z_{t}$

where

$Z_{t} \equiv \frac{N \cdot Z_{t}}{\sum_{i=1}^{N} \rho_{t, i}}$.

The above equilibrium price in (A16) gathers noise as well as information $I_{t, i}$ and so partially reveals information. By substituting (A16) into the speculator $i$ 's demand function in (A10),

$$
\begin{aligned}
& X_{t, i}=\rho_{t, i}\left(E_{t, i}\left[\tilde{P}_{t+1}\right]-P_{t}\left(E_{t}\left[\tilde{P}_{t+1}\right], z_{t}\right)\right)=\rho_{t, i} E_{t, i}\left[\tilde{r}_{t+1}\left(E_{t}\left[\tilde{P}_{t+1}\right], z_{t}\right)\right], \\
& \text { or } \\
& X_{t, i}=\rho_{t, i}\left(E_{t, i}\left[\tilde{P}_{t+1}\right]-E_{t}\left[\tilde{P}_{t+1}\right]-z_{t}\right),
\end{aligned}
$$

It is assumed that all the random variables in the model are uncorrelated. Kim and Verrecchia (1991) point out that the assumption of uncorrelation among random variables for the two-period noisy rational expectations model does not alter the results. The equilibrium price of the prior period is:

$$
P_{t-1}=E_{t-1}\left[P_{t}\right]+Z_{t-1} \text {, }
$$


By subtracting (A20) from (A16),

$$
P_{t}-P_{t-1}=E_{t}\left[\tilde{P}_{t+1}\right]-E_{t-1}\left[P_{t}\right]+z_{t}-z_{t-1}
$$

or

$$
r_{t}=E_{t}\left[\tilde{P}_{t+1}\right]-E_{t-1}\left[P_{t}\right]+z_{t}-z_{t-1} \text {. }
$$

Because by construction of the model, all the relevant information is gathered in the market price in $E_{t}\left[\tilde{P}_{t+1}\right]$ and $E_{t-1}\left[P_{t}\right]$ for the period $\mathrm{t}$ and $\mathrm{t}-1$, the expected price follows the random walk process. By letting $m_{t} \equiv E_{t}\left[\tilde{P}_{t+1}\right]$ and $m_{t-1} \equiv E_{t-1}\left[P_{t}\right]$, the above (A22) is rewritten as:

$$
r_{t}=m_{t}-m_{t-1}+z_{t}-z_{t-1}
$$

or

$$
r_{t}=f_{t}+z_{t}-z_{t-1}
$$

where

$$
m_{t}=m_{t-1}+f_{t}
$$

The above (A24) and (A25) are the typical random walk plus noise model which is used in essay $2 .^{70}$

As the demand function at $t$ is obtained in (A18) and (A19), the demand function of the prior period is:

$X_{t-1, i}=\rho_{t-1, i}\left(E_{t-1, i}\left[P_{t}\right]-P_{t-1}\left(E_{t-1}\left[P_{t}\right], z_{t-1}\right)\right)$

or

$X_{t-1, i}=\rho_{t-1, i}\left(E_{t-1, i}\left[P_{t}\right]-E_{t-1}\left[P_{t}\right]-z_{t-1}\right)$.

By subtracting (A26) from (A19),

$\frac{X_{t, i}}{\rho_{t, i}}=-P_{t}+P_{t-1}+E_{t, i}\left[\tilde{P}_{t+1}\right]-E_{t-1, i}\left[P_{t}\right]+\frac{X_{t-1, i}}{\rho_{t-1, i}}$,

or

$X_{t, i}=\rho_{t, i}\left(-r_{t}+E_{t, i}\left[\tilde{P}_{t+1}\right]-E_{t-1, i}\left[P_{t}\right]\right)+\frac{\rho_{t, i} X_{t-1, i}}{\rho_{t-1, i}}$,

From (A18),

$$
\frac{\partial X_{t, i}}{\partial E_{t, i}\left[\tilde{r}_{t+1}\right]}=\rho_{t, i}=\frac{a_{i}}{\operatorname{Var}_{t, i}\left[\tilde{P}_{t+1}\right]}>0 \text {. }
$$

From (A28),

$$
\frac{\partial X_{t, i}}{\partial r_{t}}=-\rho_{t, i}=-\frac{a_{i}}{\operatorname{Var}_{t, i}\left[\tilde{P}_{t+1}\right]}<0 \text {. }
$$

\footnotetext{
${ }^{70}$ Many books introduce the random walk plus noise model, for example, Harvey (1989), Fuller (1996) and Enders (2004). See also Hasbrouck’s papers (1991a, 1991b, 1993, 2002).
} 
The above (A29) and (A30) shows that the speculator $i$ 's demand function has the following properties:

a. The speculator $i$ 's demand is positively related with 'his' expected return $E_{t, i}\left[\tilde{r}_{t+1}\right]$.

b. The speculator $i$ 's demand gets larger if the risk-tolerance $\left(a_{i}\right)$ and the precision of his information about the return of the next period get larger. The precision is the inverse of the variance, i.e., $\frac{1}{\operatorname{Var}_{t, i}\left[\tilde{P}_{t+1}\right]}$.

c. The speculator $i$ 's demand is negatively related with the contemporaneous return $r_{t}$.

d. The speculator $i$ 's demand is negatively related with the market's expected return $E_{t}\left[\tilde{r}_{t+1}\right]$. It is because the contemporaneous return is positively related to the market's expected return as shown in (A22).

e. The speculator $i$ 's demand is negatively related with the contemporaneous market's average information $\left(f_{t}\right)$ and noise $\left(z_{t}\right)$.

f. The speculator $i$ 's demand gets smaller if the risk-tolerance and the precision of his information about the return of the next period get larger. 


\section{Appendix 3.2: Decomposition of the noise variance into the variance of order flow information and the variance of non-order flow component}

The total variance of the returns is decomposed into the informational variance and the measure of the noise variance in essay 2 based on the following first order moving average model:

$r_{\tau}=\theta_{\tau-1} u_{\tau-1}+u_{\tau}$,

where the time-varying moving average coefficient $\left(\theta_{\tau-1}\right)$ reflect the effects of old information on the current return. As mentioned in essay 2, the old information is considered as noise which is irrelevant to the expected return if the market is informationally efficient. Especially, if the returns are computed interdaily (for example, daily returns), then the information may be fully reflected when the information arrives. Hence, it is reasonable that the information does not cause any autocorrelation if the returns are computed interdaily.

However, if the returns are computed using high frequency intraday data, then information may not be fully reflected for short period of time. Hence, the intraday returns can be positively autocorrelated due to the information arrivals. The floor traders may see the short-term direction of price change which is caused by the autocorrelation by information arrivals. The floor traders also may observe the short-term direction of price change which is caused by non-information factors, such as the autocorrelated liquidity-motivated trading, bid-ask spread bounce, uninformed feedback trading, etc. This study defines the knowledge about the autocorrelation of price caused either by information or by non-information factors as the order flow information. Since essay 3 uses fifteen-minute interval returns, it is necessary to distinguish the order flow information from the non-order flow component. It is because if the traders are informed of the order flow, then there demand will be positively correlated to the factors which cause the autocorrelation. By decomposing the order flow information, the demand function of the informed traders is more properly identified. Formally, this study defines the order flow information as follows: if the current and past price changes are significantly positively correlated to the future returns, then the current and past price changes are said to have order flow information for the future returns. As stated earlier, the autocorrelation caused by the old information is reflected by the time-varying moving average coefficient $\left(\theta_{\tau-1}\right)$. The time-varying moving average coefficient is decomposed into the order flow information component and the non-order flow information component as shown below.

Consider the following future return and the autoregression of the time-varying moving average coefficients:

$r_{\tau+1}=\theta_{\tau} u_{\tau}+u_{\tau+1}$,

$\theta_{\tau}=\hat{\theta}_{\tau}\left(I_{\tau}\right)+\eta_{\tau}\left(I_{\tau+1}\right)$

where $I_{\tau}$ is the information set at the time period of $\tau$. Note that the computation of $\theta_{\tau}$

requires the information at $\tau+1 . \hat{\theta}_{\tau}\left(I_{\tau}\right)=a_{0}+\sum_{i=1}^{p} a_{i} \theta_{\tau-i}+\sum_{j=1}^{q} b_{j} \eta_{\tau-j}$ reflects the rate of the 
order flow information component of the contemporaneous innovation $\left(u_{\tau}\right)$ for the future return $\left(r_{\tau+1}\right)$, where $\hat{\theta}_{\tau}$ is obtained using the known moving average coefficients. $\eta_{\tau}$ is the non-order flow information component of the past price changes, which is orthogonal to the information set $\left(I_{\tau}\right)$ at $\tau$, i.e., $\operatorname{Cov}\left(\hat{\theta}_{\tau}\left(I_{\tau}\right), \eta_{\tau}\left(I_{\tau+1}\right)\right)=0$. Then, the (A32) can be rewritten as:

$$
r_{\tau+1}=\left(\hat{\theta}_{\tau}\left(I_{\tau}\right)+\eta_{\tau}\left(I_{\tau+1}\right)\right) u_{\tau}+u_{\tau+1} \text {, }
$$

or

$$
r_{\tau+1}=\hat{\theta}_{\tau}\left(I_{\tau}\right) u_{\tau}+\eta_{\tau}\left(I_{\tau+1}\right) u_{\tau}+u_{\tau+1} \text {, }
$$

where $u_{\tau+1}$ is the price innovation, $\hat{\theta}_{\tau}\left(I_{\tau}\right) u_{\tau}$ is the forecastable autocorrelated price change of $r_{\tau+1}$ by the order flow information, and $\eta_{\tau}\left(I_{\tau+1}\right) u_{\tau}$ is the non-forecastable price change. Consider the following simple example:

$\theta_{\tau}=a_{0}+a_{1} \theta_{\tau-1}+a_{2} \theta_{\tau-2}+b_{1} \eta_{\tau-1}+b_{2} \eta_{\tau-2}+\eta_{t}$.

Then, (A35) is rewritten as:

$r_{\tau+1}=\left(a_{0}+a_{1} \theta_{\tau-1}+a_{2} \theta_{\tau-2}+b_{1} \eta_{\tau-1}+b_{1} \eta_{\tau-2}\right) u_{\tau}+\eta_{\tau} u_{\tau}+u_{\tau+1}$.

Because the current demand will be positively related to $r_{\tau+1}$, the current demand will be also positively related to $\hat{\theta}_{\tau}\left(I_{\tau}\right)=a_{0}+a_{1} \theta_{\tau-1}+a_{2} \theta_{\tau-2}+b_{1} \eta_{\tau-1}+b_{1} \eta_{\tau-2}$. Given the contemporaneous return in (A31), it can be rewritten as:

$r_{\tau}=\left(a_{0}+a_{1} \theta_{\tau-1}\right) u_{\tau-1}+\left(\theta_{\tau-1}-a_{0}-a_{1} \theta_{\tau-1}\right) u_{\tau-1}+u_{\tau}$,

It follows that the information-motivated demand should be positively related to $\left(a_{0}+a_{1} \theta_{\tau-1}\right) u_{\tau-1}$ whereas negatively related to $\left(\theta_{\tau-1}-a_{0}-a_{1} \theta_{\tau-1}\right) u_{\tau-1}$ and $u_{\tau}$.

The noise variance of the returns shown in (10) in essay 2 is as follows:

Noise variance measure $=2 \sigma_{z}^{2}+2 \sigma_{2 f_{t-1}}=-2 \theta_{\tau-1} \sigma_{u_{t-1}}^{2}$

The measure of the noise variance is decomposed as follows:

$-2 \theta_{\tau-1} \sigma_{u_{\tau-1}}^{2}=-2\left(a_{0}+a_{1} \theta_{\tau-1}\right) \sigma_{u_{\tau-1}}^{2}-2\left(\theta_{\tau-1}-a_{0}-a_{1} \theta_{\tau-1}\right) \sigma_{u_{\tau-1}}^{2}$

where $-2\left(a_{0}+a_{1} \theta_{\tau-1}\right) \sigma_{u_{t-1}}^{2}$ is the order flow variance component and $-2\left(\theta_{\tau-1}-a_{0}-a_{1} \theta_{\tau-1}\right) \sigma_{u_{\tau-1}}^{2}$ is the non-order flow variance component of the measure of the noise variance. The absolute noise variance measure is $2\left|\theta_{\tau-1}\right| \sigma_{u_{\tau-1}}^{2}$ which represents the deviation from the random walk process of the returns. The absolute noise variance measure is decomposed into the order flow variance component and the non-order flow variance component as flows:

$2\left|\theta_{\tau-1}\right| \sigma_{u_{\tau-1}}^{2}=2\left|a_{0}+a_{1} \theta_{\tau-1}\right| \sigma_{u_{\tau-1}}^{2}+2\left|\theta_{\tau-1}-a_{0}-a_{1} \theta_{\tau-1}\right| \sigma_{u_{\tau-1}}^{2}$

where

$2\left|a_{0}+a_{1} \theta_{\tau-1}\right| \sigma_{u_{t-1}}^{2}$ is the order flow variance component of the absolute noise variance,

and 
$2\left|\theta_{\tau-1}-a_{0}-a_{1} \theta_{\tau-1}\right| \sigma_{u_{\tau-1}}^{2}$ is the non-order flow variance component of the absolute noise variance.

This study applies the $\operatorname{ARMA}(2,2)$ as shown in (A36) to obtain the intercept $a_{0}$ and the coefficient $a_{1}$ to decompose the absolute noise variance measure into the order flow variance component in (A42) and the non-order flow variance component in (A43). The determined intercepts and coefficients are as follows: $\left(a_{2}, b_{1}\right.$ and $b_{2}$ are not reported.)

\begin{tabular}{ccc}
\hline & Intercept, $a_{0}$ & Coefficient, $a_{1}$ \\
\cline { 2 - 3 } 10-year T-notes & $\mathbf{0 . 6 5 0}$ & $\mathbf{0 . 3 9 5}$ \\
Dow Jones & $\mathbf{0 . 6 7 4}$ & $\mathbf{0 . 3 5 8}$ \\
Wheat & $\mathbf{0 . 6 7 0}$ & $\mathbf{0 . 2 6 5}$ \\
Corn & $\mathbf{0 . 7 6 6}$ & $\mathbf{0 . 7 7 2}$ \\
\hline
\end{tabular}

In the regression results in Panel B of Table 3.2, the variable OrderFlowVar represents the order flow variance component of the absolute noise variance in (A42) and AbsNoiseVar represents the non-order flow variance component of the absolute noise variance in (A43). 


\section{VITA}

\section{JANG HYUNG CHO}

October 23, 1972

1999

2001

2004

2008
Incheon, Korea

Bachelor of Economics

University of Seoul

Seoul, Korea

Master of Economics

University of Seoul

Seoul, Korea

Master of Economics

University of Nebraska

Omaha, Nebraska

Doctoral Candidate in Business Administration Florida International University

Miami, Florida 